\title{
Measurement of the $W$ boson polarization in top quark decays with the ATLAS detector
}

The ATLAS collaboration

E-mail: atlas.publications@cern.ch

ABSTRACT: This paper presents measurements of the polarization of $W$ bosons in top quark decays, derived from $t \bar{t}$ events with missing transverse momentum, one charged lepton and at least four jets, or two charged leptons and at least two jets. Data from $p p$ collisions at a centre-of-mass energy of $7 \mathrm{TeV}$ were collected with the ATLAS experiment at the LHC and correspond to an integrated luminosity of $1.04 \mathrm{fb}^{-1}$. The measured fractions of longitudinally, left- and right-handed polarization are $F_{0}=0.67 \pm 0.07, F_{\mathrm{L}}=0.32 \pm 0.04$ and $F_{\mathrm{R}}=0.01 \pm 0.05$, in agreement with the Standard Model predictions. As the polarization of the $W$ bosons in top quark decays is sensitive to the $W t b$ vertex Lorentz structure and couplings, the measurements were used to set limits on anomalous contributions to the $W t b$ couplings.

KEYworDS: Hadron-Hadron Scattering 


\section{Contents}

1 Introduction 1

2 Data and simulation samples $\quad 3$

2.1 Data sample 3

2.2 Signal and background modelling 3

3 Event selection $\quad 4$

3.1 Physics object definition 4

3.2 Single-lepton channels 5

3.3 Dilepton channels 6

4 Measurement strategies $\quad 8$

4.1 Measurement using the template method 8

$\begin{array}{lll}4.2 & \text { Measurement using the angular asymmetries } & 11\end{array}$

5 Systematic uncertainties $\quad \mathbf{1 2}$

$\begin{array}{lll}5.1 & \text { Sources of systematic uncertainty } & 12\end{array}$

$\begin{array}{lll}\text { 5.1.1 Signal and background modelling } & 12\end{array}$

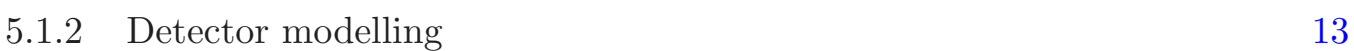

6 Results $\quad 15$

$\begin{array}{lll}\text { 6.1 Combination } & 16\end{array}$

$\begin{array}{ll}\text { 6.2 Constraints on the } W t b \text { vertex structure } & 18\end{array}$

$\begin{array}{lll}7 & \text { Conclusions } & 20\end{array}$

$\begin{array}{ll}\text { The ATLAS collaboration } & 25\end{array}$

\section{Introduction}

Discovered in 1995 by the CDF and DØ experiments [1,2], the top quark is the heaviest known fundamental particle, with a mass of $173.2 \pm 0.9 \mathrm{GeV}[3]$. Measurements of top quark properties play an important role in testing the Standard Model and its possible extensions. One particular test is the study of the $W t b$ vertex Lorentz structure and couplings, which can be probed by measuring the polarization of $W$ bosons produced in top quark decays.

At the LHC, top quarks are produced mainly in pairs via the strong interaction and are predicted to decay via the electroweak interaction into a $W$ boson and a bottom quark with a nearly $100 \%$ branching fraction. Events with $t \bar{t}$ pairs can thus be classified according to the decay of the two produced $W$ bosons. Each boson can decay either into 
a quark-antiquark pair or into a charged lepton and a neutrino. The single-lepton and dilepton topologies, both considered in the analyses presented in this paper, have one and two isolated charged leptons in the final state. Only electrons and muons, including those from $\tau$ decays, are considered here.

The $W t b$ vertex is defined by the electroweak interaction and has a $(V-A)$ structure where $V$ and $A$ are the vector and axial-vector contributions to the vertex. Since the $W$ bosons are produced as real particles in top quark decays, their polarization can be longitudinal, left-handed or right-handed. The fractions of events with a particular polarization, $F_{0}, F_{\mathrm{L}}$ and $F_{\mathrm{R}}$, are referred to as helicity fractions. They are predicted in next-to-nextto-leading-order (NNLO) QCD calculations to be $F_{0}=0.687 \pm 0.005, F_{\mathrm{L}}=0.311 \pm 0.005$, $F_{\mathrm{R}}=0.0017 \pm 0.0001$ [4]. These fractions can be extracted from measurements of the angular distribution of the decay products of the top quark. The angle $\theta^{*}$ is defined as the angle between the momentum direction of the charged lepton from the decay of the $W$ boson and the reversed momentum direction of the $b$-quark from the decay of the top quark, both boosted into the $W$ boson rest frame [5]. The angular distribution is:

$$
\frac{1}{\sigma} \frac{\mathrm{d} \sigma}{\mathrm{d} \cos \theta^{*}}=\frac{3}{4}\left(1-\cos ^{2} \theta^{*}\right) F_{0}+\frac{3}{8}\left(1-\cos \theta^{*}\right)^{2} F_{\mathrm{L}}+\frac{3}{8}\left(1+\cos \theta^{*}\right)^{2} F_{\mathrm{R}} .
$$

All previous measurements of the helicity fractions, performed by the CDF and DØ Collaborations [6-8] at the Tevatron, are in agreement with Standard Model predictions.

Information about the polarization of the $W$ bosons can also be obtained through complementary observables, such as the angular asymmetries, $A_{+}$and $A_{-}$, defined as:

$$
A_{ \pm}=\frac{N\left(\cos \theta^{*}>z\right)-N\left(\cos \theta^{*}<z\right)}{N\left(\cos \theta^{*}>z\right)+N\left(\cos \theta^{*}<z\right)},
$$

with $z= \pm\left(1-2^{2 / 3}\right)$ for $A_{ \pm}$, allowing the dependence on $F_{\mathrm{L}}$ and $F_{\mathrm{R}}$ to cancel, respectively. The asymmetries can be related to the helicity fractions by a simple system of equations $[9,10]$. In the Standard Model, the NNLO values for these asymmetries are $A_{+}=0.537 \pm 0.004$ and $A_{-}=-0.841 \pm 0.006$ [4].

In the presence of anomalous $W t b$ couplings the helicity fractions and angular asymmetries depart from their Standard Model values [5, 10]. In effective field theories, dimensionsix operators can be introduced which modify the $W t b$ vertex [11-13]. Coefficients controlling the strength of these operators can be constrained by measurements of the helicity fractions or the angular asymmetries.

This paper describes measurements of the $W$ boson polarization in top quark decays and the constraints on the $W t b$ vertex structure based on a data set recorded with the ATLAS detector between March and June 2011 and corresponding to an integrated luminosity of $1.04 \mathrm{fb}^{-1}$. The helicity fractions were measured using two different methods. The first compares the observed $\cos \theta^{*}$ distribution with templates for different $W$ boson helicity states obtained from simulation. The second method extracts angular asymmetries from an unfolded $\cos \theta^{*}$ spectrum corrected for background contributions. Limits on anomalous couplings, generated by the aforementioned dimension-six operators, were set using the combined result from the two measurements. 
This paper is structured as follows. Data samples and simulated samples modelling signal and background processes are described in section 2, followed by a summary of the event selection in section 3. The analysis strategies can be found in section 4 . Sources of systematic uncertainty are discussed in section 5 , the results are summarized in section 6 and section 7 concludes the paper.

\section{Data and simulation samples}

The ATLAS detector [14] at the LHC covers nearly the entire solid angle around the collision point. It consists of an inner tracking detector surrounded by a thin superconducting solenoid, electromagnetic and hadronic calorimeters, and an external muon spectrometer incorporating three large superconducting toroid magnet assemblies. A three-level trigger system, designed to reduce the event rate from $40 \mathrm{MHz}$ to about $200 \mathrm{~Hz}$, is used to select events of interest.

\subsection{Data sample}

Data from $p p$ collisions at a centre-of-mass energy of $7 \mathrm{TeV}$ were collected using singlelepton triggers with transverse momentum thresholds of $20 \mathrm{GeV}$ for electrons and $18 \mathrm{GeV}$ for muons. The size of the data sample corresponds to an integrated luminosity of $1.04 \pm$ $0.04 \mathrm{fb}^{-1}[15,16]$.

\subsection{Signal and background modelling}

Signal and most background processes were modelled by Monte Carlo (MC) simulation. The signal process was simulated using two different generators. The leading order (LO) Monte Carlo generator Protos $[10,17]$ was used with the CTEQ6L1 sets of parton distribution functions (PDFs) [18] to generate three samples assuming $F_{0}=1, F_{\mathrm{L}}=1$ and $F_{\mathrm{R}}=1$. This was achieved by choosing appropriate values for anomalous $W t b$ couplings, as described in refs. [9, 10]. The output was interfaced to Pyтнia [19] to simulate parton showers and hadronization. In addition, the next-to-leading-order (NLO) generator MC@NLO [20-22] was used with the CTEQ6.6 PDF set [23] for studies of systematic differences in the top quark production and decay modelling.

The $W$ and $Z$ boson production in association with multiple jets was simulated using the ALPGEn generator [24] and the CTEQ6L1 PDF set. Tree-level matrix elements with up to five final-state partons were included. The standard ALPGEN matching scheme was used to remove overlaps between the $n$ and $n+1$ parton samples. Heavy flavour samples containing $W b \bar{b}, W c \bar{c}, W c$ and $Z b \bar{b}$ events were simulated separately. The $Z+$ jets samples were generated with dileptons in the invariant mass range $10<m_{\ell \ell}<2000 \mathrm{GeV}$. Diboson processes were simulated using HERWIG [25]. Single top quark production was simulated using MC@NLO, invoking the 'diagram removal scheme' [26] to remove overlaps between the single top quark and $t \bar{t}$ final states. An additional sample of $W+$ jets events was generated with SHERPA [27] and used to study systematic uncertainties. Details are described in section 5. Apart from the Protos $t \bar{t}$ samples, all events were hadronized with 
Herwig using Jimmy [28] for the underlying event model. Different underlying event tunes were used, depending on the hadronization program used (i.e. Pythia or Herwig) [29].

Additional MC samples were used for the evaluation of systematic uncertainties. These were generated with the ACERMC [30] and Powheg [31, 32] generators interfaced to PythiA for hadronization. They are described in section 5. A top quark mass of $172.5 \mathrm{GeV}$ was assumed for all signal samples if not stated otherwise. Additional MC@NLO samples were generated assuming different top quark masses.

All simulated events contain multiple $p p$-interactions and contain pileup contributions corresponding to a bunch spacing of 50 ns. These simulated events were re-weighted such that the average number of interactions per proton-proton bunch crossing was the same in data and MC simulation. The average number of interactions per event was roughly six. All samples were processed by the detector and trigger simulation after event generation $[33,34]$, and subjected to the same reconstruction algorithms as the data.

The cross-section of simulated $t \bar{t}$ samples was normalized to $164.6 \mathrm{pb}$, the value obtained from approximate NNLO calculations [35-37], and consistent with recent measurements [38]. While the $Z+$ jets normalization was obtained from the NNLO QCD crosssection calculations in the single-lepton channels, a data-driven estimate for the normalization was used for the dilepton channels [38]. The diboson with jets production was rescaled to match NLO calculations of the inclusive cross-sections.

For the single-lepton analysis, the multijet production background, where an electron or muon originates from hadron decay or instrumental background, was estimated from data as described in refs. [39, 40]. The $W+$ jets background was obtained from simulation, except that its normalization was derived from data, from a study of the asymmetry in the production of $W^{+}$and $W^{-}$bosons [40]. For the dilepton analysis, background contributions from $W+$ jets, single-lepton $t \bar{t}$ and single top quark production were estimated using the method described in ref. [38]. In the single-lepton and dilepton analyses this source of background is labelled as "misidentified leptons".

\section{Event selection}

\subsection{Physics object definition}

The reconstruction and identification of electrons, muons, jets and the magnitude of the missing transverse momentum $\left(E_{\mathrm{T}}^{\mathrm{miss}}\right)$ used in the analyses presented here followed the criteria employed for the measurement of the $t \bar{t}$ production cross-section [39]. More details, including a description of the trigger and the vertex requirements, can be found in ref. [40].

The $t \bar{t}$ events with at least one isolated charged lepton (electron or muon) in the final state were considered as signal. Events with $\tau$ leptons decaying into muons or electrons in the final state were also considered to be part of the signal.

All the considered events were required to fulfil general event quality criteria and to have a well-defined primary vertex with at least five associated tracks. Reconstructed electrons were required to have $E_{\mathrm{T}}>25 \mathrm{GeV}$ and $|\eta|<2.47$ excluding $1.37<|\eta|<1.52$, 
while muons were required to have $p_{\mathrm{T}}>20 \mathrm{GeV}$ and $|\eta|<2.5 .{ }^{1}$ Jets were reconstructed with the anti- $k_{t}$ algorithm [41] with a radius parameter equal to 0.4 . They were required to have $p_{\mathrm{T}}>25 \mathrm{GeV}$ and $|\eta|<2.5$. For electrons, the energy not associated to the electron cluster but contained in a cone of $\Delta R=\sqrt{(\Delta \phi)^{2}+(\Delta \eta)^{2}}=0.2$ around it was required not to exceed $3.5 \mathrm{GeV}$. For muons, the sum of track transverse momenta and the total energy deposited in a cone of $\Delta R=0.3$ around the muon were both required to be less than $4 \mathrm{GeV}$. Muons reconstructed within a $\Delta R=0.4$ cone of a jet satisfying $p_{\mathrm{T}}>20 \mathrm{GeV}$ were removed to reduce the contamination caused by muons from hadron decays within jets. Subsequently, jets within $\Delta R=0.2$ of an electron candidate were removed to avoid double counting, which can occur because electron clusters are also reconstructed as jets.

A combination of two algorithms was used for $b$-jet identification ( $b$-tagging). They are based on reconstructed secondary vertices and the impact parameter significances of tracks within jets. The chosen working point resulted in a $b$-tagging efficiency of $70 \%$ for jets originating from $b$-quarks in a sample of simulated $t \bar{t}$ events and a light-quark jet rejection factor of about 100 [42].

\subsection{Single-lepton channels}

The single-lepton $t \bar{t}$ signal is characterized by a high- $p_{\mathrm{T}}$ isolated charged lepton and missing transverse momentum from the neutrino, from the leptonically decaying $W$ boson, two light-quark jets from the hadronically decaying $W$ boson and two $b$-quark jets. The two channels with either an electron or a muon in the final state are referred to as single-electron and single-muon channels. The following event selection requirements were applied:

- the appropriate single-electron or single-muon trigger had fired;

- events were required to contain exactly one isolated electron or muon;

- in the single-electron channel, $E_{\mathrm{T}}^{\text {miss }}>35 \mathrm{GeV}$ and $m_{\mathrm{T}}(W)>25 \mathrm{GeV}$ were required ${ }^{2}$ while in the single-muon channel the criteria were $E_{\mathrm{T}}^{\mathrm{miss}}>20 \mathrm{GeV}$ and $E_{\mathrm{T}}^{\mathrm{miss}}+$ $m_{\mathrm{T}}(W)>60 \mathrm{GeV}$;

- events were required to have at least four jets, with at least one of them being tagged as a $b$-jet.

The numbers of events expected after all selection requirements are shown in table 1 for the single-electron and single-muon channels, together with the observed numbers of events in the data sample. The single-electron channel contains significantly fewer events than the single-muon channel due to the more stringent requirements on the lepton $E_{\mathrm{T}}$ $\left(p_{\mathrm{T}}\right), E_{\mathrm{T}}^{\mathrm{miss}}$ and $m_{\mathrm{T}}(W)$. The observed numbers of events are in agreement with those

\footnotetext{
${ }^{1}$ ATLAS uses a right-handed coordinate system with its origin at the nominal interaction point (IP) in the centre of the detector and the $z$-axis along the beam pipe. The $x$-axis points from the IP to the centre of the LHC ring, and the $y$-axis points upward. Cylindrical coordinates $(r, \phi)$ are used in the transverse plane, $\phi$ being the azimuthal angle around the beam pipe. The pseudorapidity is defined in terms of the polar angle $\theta$ as $\eta=-\ln \tan (\theta / 2)$.

${ }^{2}$ Here $m_{\mathrm{T}}(W)$ is the $W$ boson transverse mass, reconstructed as $\sqrt{2 p_{\mathrm{T}}^{\ell} p_{\mathrm{T}}^{\nu}\left[1-\cos \left(\phi^{\ell}-\phi^{\nu}\right)\right]}$ where the measured missing transverse momentum provides the neutrino information.
} 


\begin{tabular}{|lcr|}
\hline Process & Single electron & Single muon \\
\hline$t \bar{t}$ & $4400 \pm 1100$ & $6500 \pm 1400$ \\
$W+$ jets & $900 \pm 700$ & $1400 \pm 1000$ \\
$Z+$ jets & $120 \pm \quad 90$ & $140 \pm \quad 90$ \\
Diboson & $14 \pm \quad 12$ & $22 \pm \quad 12$ \\
Single top & $260 \pm \quad 90$ & $360 \pm 110$ \\
Misidentified leptons & $220 \pm 220$ & $500 \pm 500$ \\
\hline Total predicted & $5900 \pm 1300$ & $9000 \pm 1800$ \\
\hline Data & 5830 & 9121 \\
\hline
\end{tabular}

Table 1. Event yields in the single-electron and single-muon channels after the event selection. The table shows the expected number of events including their estimated total uncertainty as well as the number of events observed in the data sample.

expected, with purities of $75 \%$ and $72 \%$ for the single electron and single muon channels. The systematic uncertainty discussed in section 5 dominates the error on the event yields, with jet energy scale, signal and background modelling, and $b$-tagging being the dominant sources of uncertainty. Example distributions in data are compared with the Standard Model predictions in figure 1. The data are described well by the expected distributions.

\subsection{Dilepton channels}

The dilepton $t \bar{t}$ signal is characterized by two high- $p_{\mathrm{T}}$ isolated charged leptons, missing transverse momentum corresponding to the undetected neutrinos from the two leptonically decaying $W$ bosons, and two $b$-quark jets. The three channels are referred to as $e e, \mu \mu$ and $e \mu$ channels. The following event selection requirements were applied:

- the single-electron trigger had fired for the ee channel, the single-muon trigger had fired for the $\mu \mu$ channel and either of these triggers had fired for the $e \mu$ channel;

- events were required to contain exactly two oppositely charged and isolated leptons $(e e, \mu \mu$ or $e \mu)$;

- at least two jets, with at least one of them being $b$-tagged, were required;

- in order to avoid the low-mass Drell-Yan background region, events were required to have $m_{\ell \ell}>15 \mathrm{GeV}$;

- in the $e e$ and $\mu \mu$ channels, the missing transverse momentum had to satisfy $E_{\mathrm{T}}^{\text {miss }}>$ $40 \mathrm{GeV}$, and the invariant mass of the two leptons had to differ by at least $10 \mathrm{GeV}$ from the $Z$ boson mass, i.e. $\left|m_{\ell \ell}-m_{Z}\right|>10 \mathrm{GeV}$, with $m_{Z}=91 \mathrm{GeV}$, in order to suppress backgrounds from $Z+$ jets events and events containing misidentified leptons; 

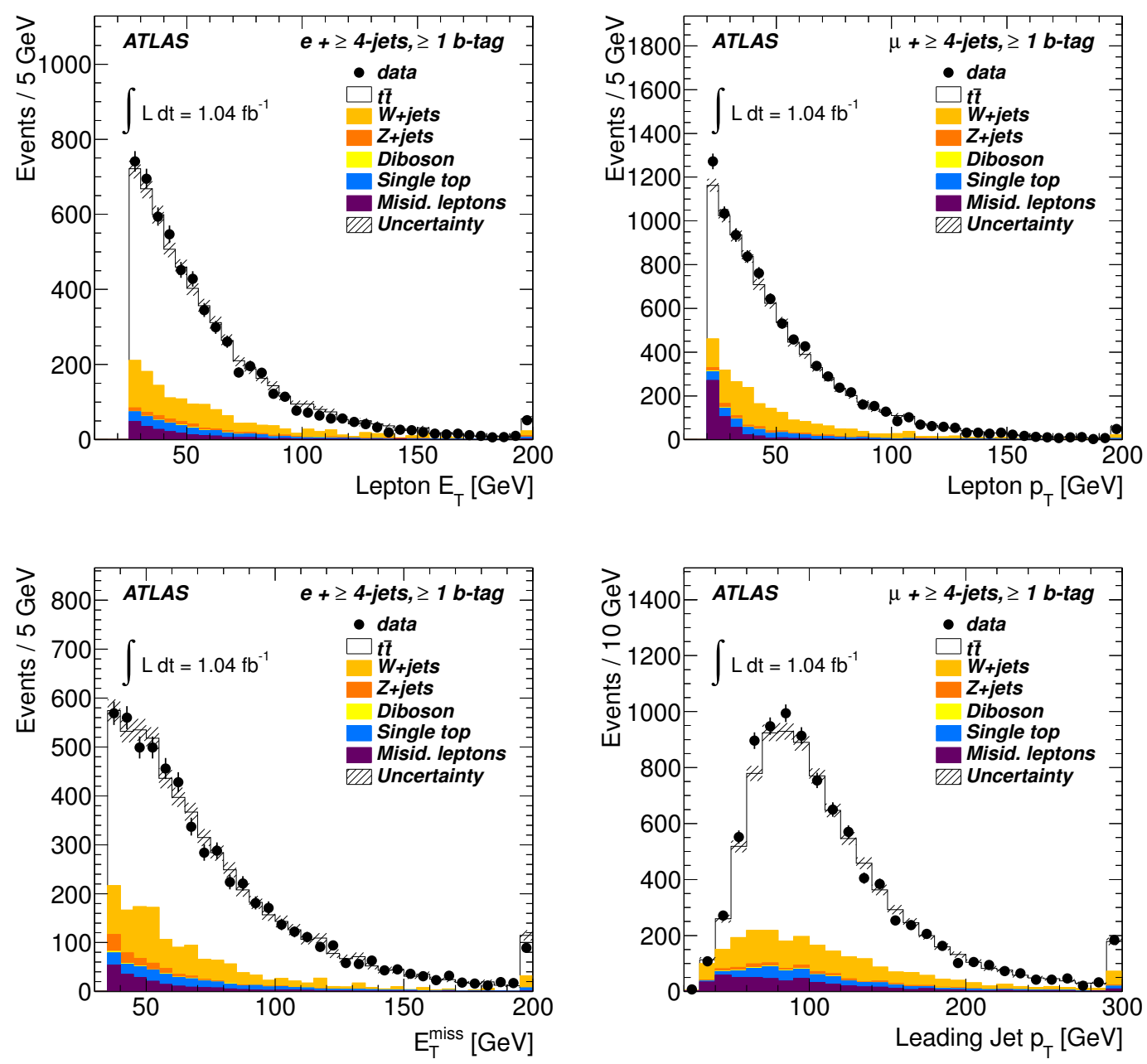

Figure 1. The top row shows (left) the $E_{\mathrm{T}}$ spectrum of the electron in the single-electron channel and (right) the $p_{\mathrm{T}}$ spectrum of the muon in the single-muon channel. The bottom row shows (left) the missing transverse momentum in the single-electron channel and (right) the $p_{\mathrm{T}}$ spectrum of the leading jet in the single-muon channel. The error band shows the statistical uncertainty from the Monte Carlo simulation. The last bin in each distribution includes the overflow.

- in the $e \mu$ channel, no $E_{\mathrm{T}}^{\text {miss }}$ or $Z$ boson mass veto cuts were applied; however, the event $H_{\mathrm{T}}$, defined as the scalar sum of the transverse energies of the two charged leptons and all selected jets, had to satisfy $H_{\mathrm{T}}>130 \mathrm{GeV}$ to suppress backgrounds from $Z+$ jets production;

- events were required to pass the $t \bar{t}$ reconstruction criteria, as described in section 4.1.

The numbers of events expected after all selection requirements are shown in table 2 for the three dilepton channels, together with the observed numbers of events in the data sample. The observed numbers of events is in agreement with those expected, with a purity of $94 \%$ for all three channels. The systematic uncertainty discussed in section 5 


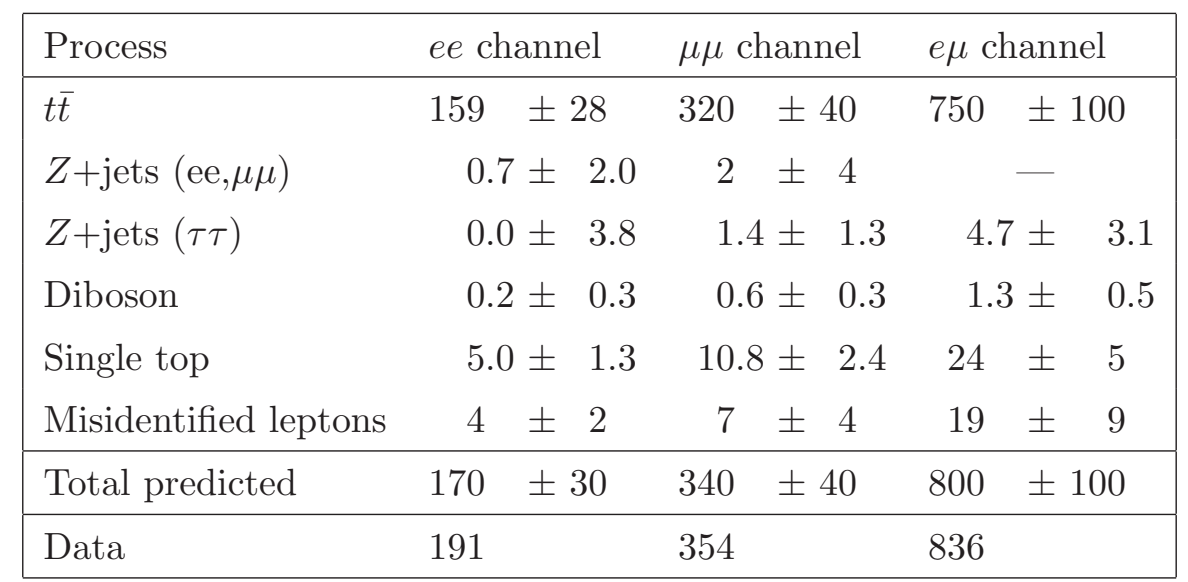

Table 2. Event yields in the dilepton channels after the event selection and event reconstruction. The table shows the expected number of events including their estimated total uncertainty as well as the number of events observed in the data sample.

dominates the error on the event yields, with jet energy scale, jet energy resolution, signal and background modelling, and $b$-tagging being the dominant sources of uncertainty. Example distributions in data are compared with the Standard Model predictions in figure 2. The data are described well by the expected distributions.

\section{Measurement strategies}

The two methods to extract the helicity fractions are described below. The analysis was carried out for the single-electron, single-muon, ee, $e \mu$ and $\mu \mu$ channels separately. The reconstruction of $t \bar{t}$ events in the single-lepton channels differs for the two methods: the template method relies more strongly on the structure of the $\cos \theta^{*}$ distribution than the calculation of the angular asymmetries. It thus relies on an improved estimate of the particle energies. A common reconstruction method was used in the dilepton channels. The results of the analyses and their combination are described in section 6 .

\subsection{Measurement using the template method}

Templates for different signal and background processes were fitted to the observed $\cos \theta^{*}$ distributions based on events reconstructed with a kinematic fit in the single-lepton channels $[40,43]$. The fit was based on a likelihood which took into account the Breit-Wigner forms of the lineshapes of the top quark and the $W$ boson as well as the energy resolution of the measurements of the jets and the charged leptons. The missing transverse momentum was identified with the $x$ - and $y$-components of the neutrino momentum. The top quark mass was fixed to $172.5 \mathrm{GeV}$, and the mass of the $W$ boson was fixed to $80.4 \mathrm{GeV}$. The likelihood was maximized with respect to the energies of the final state quarks for each association of jets to quarks. The permutations were weighted according to the $b$ tagging information and the weights were derived from the efficiency and mis-tag rate of the $b$-tagger. The permutation with the largest value of the likelihood was used in the 

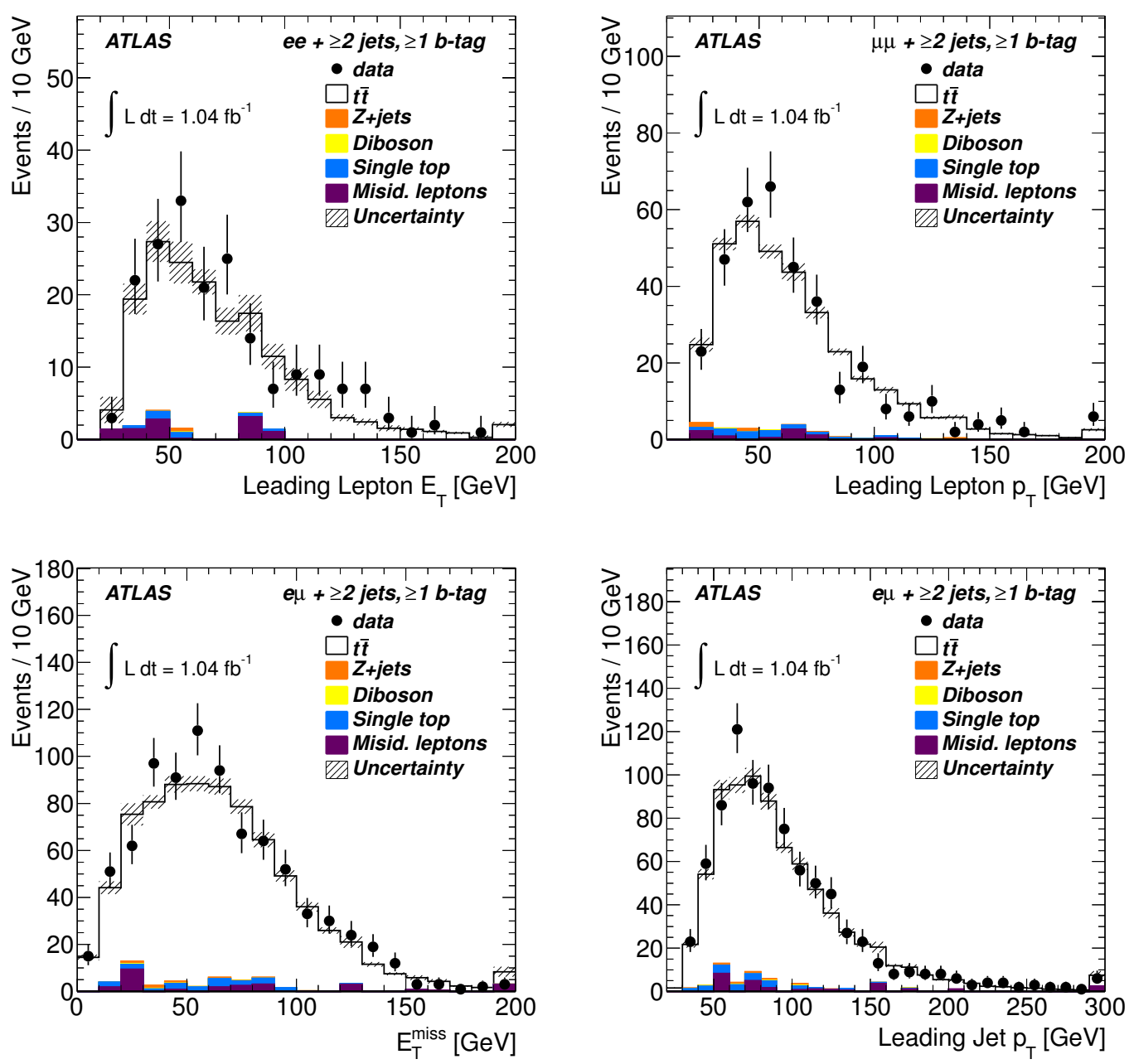

Figure 2. The top row shows (left) the $E_{\mathrm{T}}$ spectrum of the leading electron in the ee channel and (right) the $p_{\mathrm{T}}$ spectrum of the leading muon in the $\mu \mu$ channel. The bottom row shows (left) the missing transverse momentum in the $e \mu$ channel and (right) the $p_{\mathrm{T}}$ spectrum of the leading jet in the $e \mu$ channel. The error band shows the statistical uncertainty from the Monte Carlo simulation. The last bin in each distribution includes the overflow.

following steps of the analysis. The overall efficiency for the reconstruction of the correct event topology was found to be $74 \%$ in simulated $t \bar{t}$ events. Only those events where four jets and a lepton are matched to partonic particles, corresponding to roughly $30 \%$ of the events, were considered for the efficiency computation.

For the dilepton channels, the $t \bar{t}$ system was reconstructed by solving a set of six independent equations:

$$
\begin{aligned}
& p_{x}^{\nu_{1}}+p_{x}^{\nu_{2}}=E_{x}^{\text {miss }} ; \quad p_{y}^{\nu_{1}}+p_{y}^{\nu_{2}}=E_{y}^{\text {miss }} ; \quad\left(p_{\ell_{1}}+p_{\nu_{1}}\right)^{2}=m_{W}^{2} ; \\
& \left(p_{\ell_{2}}+p_{\nu_{2}}\right)^{2}=m_{W}^{2} ; \quad\left(p_{W_{1}}+p_{j_{1}}\right)^{2}=m_{t}^{2} ; \quad\left(p_{W_{2}}+p_{j_{2}}\right)^{2}=m_{t}^{2} .
\end{aligned}
$$

$E_{x}^{\text {miss }}$ and $E_{y}^{\text {miss }}$ represent the $x$ - and $y$-components of the missing transverse momentum, 
$p_{\ell_{1}}$ and $p_{\ell_{2}}\left(p_{j_{1}}\right.$ and $p_{j_{2}}, p_{\nu_{1}}$ and $\left.p_{\nu_{2}}\right)$ correspond to the four-momenta of the two charged leptons (jets, neutrinos). Here, $m_{W}$ and $m_{t}$ are the $W$ boson and top quark masses, respectively. For events with only one $b$-tagged jet, this jet and the non-tagged one with the highest $p_{\mathrm{T}}$ were taken as $b$-jets from the top quark decays. If at least two $b$-tagged jets are present, the two $b$-tagged jets with the largest $p_{\mathrm{T}}$ in the event were used. The pairing of the jets with the charged leptons was based on the minimization of the sum of the invariant masses $m_{\ell_{1} j_{1}}$ and $m_{\ell_{2} j_{2}}$. Simulations show that this criterion gives the correct pairing in $68 \%$ of the events. Up to four solutions can be found to the Equations 4.1. The solution with the minimum product of neutrino transverse momenta was chosen and is motivated by the low $p_{\mathrm{T}}$-spectrum of the neutrinos. In case no solution was found, the top quark mass parameter was varied in the $157.5-187.5 \mathrm{GeV}$ range, to try to find one or more solutions. In case a range of top masses provided a solution, that with the top quark mass parameter closest to $172.5 \mathrm{GeV}$ was taken. Again, multiple ambiguities were resolved by taking the solution with the minimum product of neutrino transverse momenta. If still no solution was found, the second pairing of jets and charged leptons was used. If no solution was found after this procedure the event was discarded. This happened in about $25 \%$ of the events in data and in simulated $t \bar{t}$ events.

In the single-lepton channels, the following six processes were used as templates: three different helicity state signal processes, the background from events with misidentified leptons, the $W+$ jets contribution and the sum of all other sources of background. The three signal templates were derived from simulation and include single-lepton and dilepton events which pass the event selection. These distributions are shown in figure 3 . The $W+$ jets template was obtained from simulation. The template for events with misidentified leptons was obtained from data. Other processes contributing to the observed spectrum include single top quark, diboson and $Z+$ jets production and were summed in a single template obtained from simulation.

In the dilepton channels, templates for the three different helicity state signal processes were used as well as a single template representing the different background contributions, namely single top quark production, processes with misidentified leptons, production of $Z$ bosons with additional jets and diboson production. All templates were obtained from simulation, except the misidentified leptons template, which was obtained from data.

A binned likelihood fit was used to estimate the expected number of events contributing to the distribution, assuming independent Poisson-distributed fluctuations in each bin. The number of events expected in the $i$ th bin, $\lambda_{i}$, is given as:

$$
\lambda_{i}=\sum_{h=-1,0,+1} \lambda_{i}^{h} \cdot \epsilon^{h}+\sum_{j=1}^{N_{\mathrm{bkg}}} \lambda_{i}^{j},
$$

where the first term describes the expected number of signal events at particle level with defined helicity $h$. These are reduced by reconstruction efficiencies and acceptances summarized by the factors $\epsilon^{h}$ estimated from simulation. The second term describes the expected number of events from background processes and $N_{\mathrm{bkg}}$ is the number of background templates. The contributions of the various background templates were constrained according 

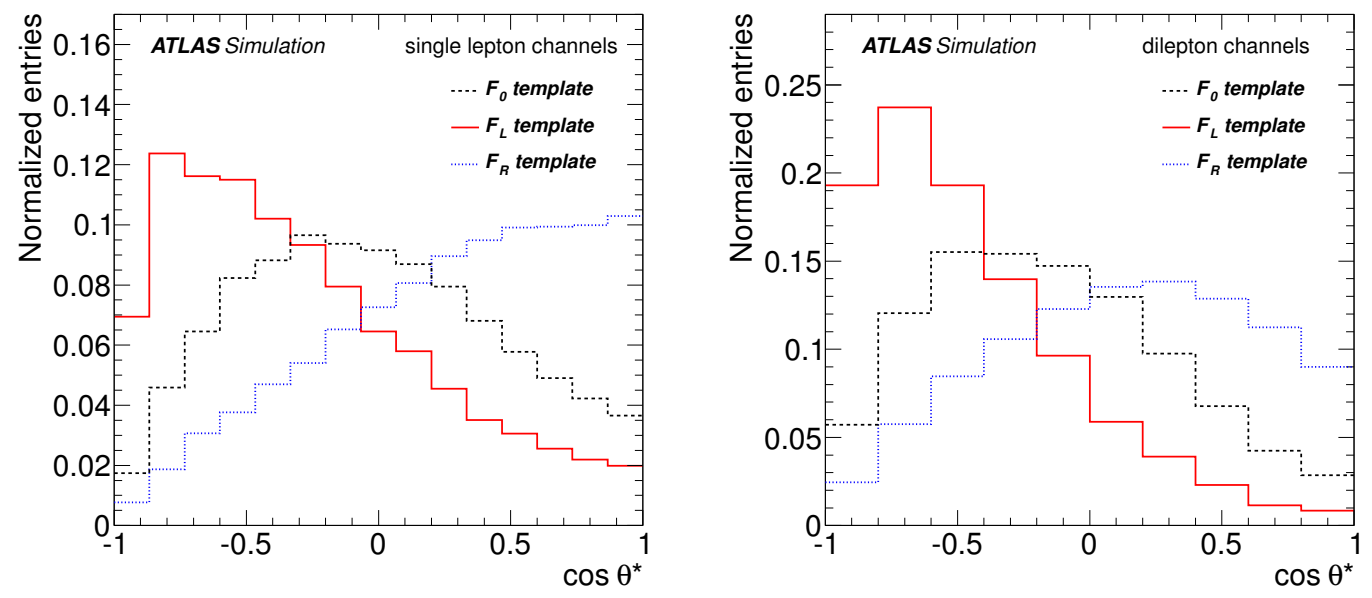

Figure 3. Distributions of $\cos \theta^{*}$ for each of the three simulated signal templates. The templates for the combined (left) single-lepton and (right) dilepton channels are shown.

to the central values and uncertainties given in table 1 and table 2 . The uncertainties were assumed to be uncorrelated.

\subsection{Measurement using the angular asymmetries}

The angular asymmetries were measured using the $\cos \theta^{*}$ distribution obtained by reconstructing the events with a $\chi^{2}$ minimization technique in the single-lepton channels [44]. The $\chi^{2}$ was defined according to:

$$
\chi^{2}=\frac{\left(m_{\ell \nu j_{a}}-m_{t}\right)^{2}}{\sigma_{t}^{2}}+\frac{\left(m_{j_{b} j_{c} j_{d}}-m_{t}\right)^{2}}{\sigma_{t}^{2}}+\frac{\left(m_{\ell \nu}-m_{W}\right)^{2}}{\sigma_{W}^{2}}+\frac{\left(m_{j_{c} j_{d}}-m_{W}\right)^{2}}{\sigma_{W}^{2}}
$$

where $m_{t}=172.5 \mathrm{GeV}, m_{W}=80.4 \mathrm{GeV}, \sigma_{t}=14 \mathrm{GeV}$ and $\sigma_{W}=10 \mathrm{GeV}$ are the expected top quark and $W$ boson mass resolutions, $\ell$ represents the selected electron or muon, $m_{\ell \nu}$ is the invariant mass of the electron (muon) and the neutrino, and $j_{a, b, c, d}$ corresponds to all possible combinations of four jets among all selected jets in the event (with $m_{\ell \nu j_{a}}, m_{j_{b} j_{c} j_{d}}$ and $m_{\ell \nu j_{a}}$ being the corresponding invariant masses). The neutrino was reconstructed using the missing transverse energy, with the longitudinal component of the neutrino momentum $\left(p_{z}^{\nu}\right)$ allowed to vary. The solution corresponding to the minimum $\chi^{2}$ value is chosen. No $b$-tagging information was used in resolving the ambiguities in assigning jets.

The method described in the previous subsection was used to reconstruct dilepton $t \bar{t}$ events.

For the measurement of the angular asymmetries, $A_{+}$and $A_{-}$, the $\cos \theta^{*}$ distribution was divided into four non-uniform bins, which were used to count the number of events above and below $z= \pm\left(1-2^{2 / 3}\right)$, as defined in Equation 1.2. A background subtraction in the observed $\cos \theta^{*}$ distribution was performed. Subsequently, the following steps were applied iteratively: the number of reconstructed events above and below $\cos \theta^{*}=z$ were counted in data for each asymmetry and correction factors were evaluated by comparing 
the Standard Model expectation with the reconstructed number of simulated $t \bar{t}$ events. These factors allowed corrections to be made for event selection and reconstruction effects. The obtained angular asymmetries were then converted into $W$ boson helicity fractions and these values were used to re-derive the $\cos \theta^{*}$ distribution and evaluate new correction factors. The procedure was repeated until the method converged, i.e. until the differences between the output observables and the input hypothesis at the previous iteration step were below $0.5 \%$. Closure tests were performed using Monte Carlo samples with different helicity fractions and no bias was observed.

\section{Systematic uncertainties}

\subsection{Sources of systematic uncertainty}

Several sources of systematic uncertainty were taken into account in the analyses presented here. These were categorized into the modelling of the signal and background processes, and the detector modelling. The impact of the systematic uncertainties on the final results is summarized in table 3 .

\subsubsection{Signal and background modelling}

The signal process was modelled with different Monte Carlo generators. Sources of systematic uncertainty considered here were the choice of generator and parton shower model, the choice of parton distribution functions, the assumed top quark mass and the choice of parameters which control the amount of initial and final state radiation. Predictions from the MC@NLO and POWHEG generators were compared. The parton showering was tested by comparing two Powheg samples interfaced to Herwig and Pythia, respectively. The amount of initial and final state radiation was varied by modifying parameters in ACERMC interfaced to PythiA. The parameters were varied in a range comparable with those used in the Perugia Soft/Hard tune variations [45]. The impact of the choice of parton distribution functions was studied using the reweighting procedure described in ref. [44]. MC@NLO samples were generated assuming different top quark masses and their predictions were compared. The observed differences in the results were scaled to variations of $0.9 \mathrm{GeV}$ in the top quark mass according to the uncertainty on its Tevatron average value [3]. The impact of different models of colour reconnection was studied by comparing samples simulated with ACERMC using the Perugia 2010 tune with and without colour reconnection [45] as well as the tune A-Pro and ACR-Pro [46, 47].

Background processes were either modelled by simulation or were estimated in auxiliary measurements. The number of events with misidentified leptons was estimated in data for each channel [40] and the uncertainty on the normalization was estimated to be $50 \%$ before and $100 \%$ after the $b$-tagging requirement. The normalization of $W+$ jets processes was estimated from supplementary measurements using the asymmetric production of positively and negatively charged $W$ bosons. The uncertainty was estimated using Berends-Gielescaling [48] which yielded $48 \%$ for events with four jets and increased with the jet multiplicity by $24 \%$ per additional jet [39]. Systematic uncertainties on the shapes of the $W+$ jets distributions were assigned based on samples with different simulation parameters such as 
the minimum transverse momentum of the parton and the functional form of the factorization scale in ALPGEN. Scaling factors correcting the fraction of heavy flavour contributions in simulated $W+$ jets samples were estimated in auxiliary measurements described in ref. [40]. The uncertainties were $76 \%$ for $W b \bar{b}+$ jets and $W c \bar{c}+$ jets contributions, and $35 \%$ for $W c+$ jets contributions. The uncertainty on the normalization of $Z+$ jets events was estimated using Berends-Giele-scaling. The uncertainties in the normalization were $48 \%$ for events with four jets and increased with the jet multiplicity by $24 \%$ per additional jet. A systematic uncertainty in the shape was accounted for by comparing simulated samples generated with ALPGEN and SHeRPA. The uncertainty on the normalization of the small background contributions from single top quark and diboson production was estimated to be about $10 \%$ (depending on the channel) and $5 \%$, respectively. The former estimate was based on the difference between the predictions from MC@NLO and MCFM [49], whereas the latter comes from scale and PDF uncertainties evaluated with MCFM.

For the template method, the bin content in each template was varied according to a Poisson distribution to estimate the impact of the finite Monte Carlo sample size used. For the measurement of the angular asymmetries, the $t \bar{t}$ reconstruction parameters were varied. In the single-lepton channels, the top quark and $W$ boson mass resolutions used in the $\chi^{2}$ definition were changed by $25 \%$. For the dilepton channels, the assumed top quark mass window used in the kinematic equations was reduced by $50 \%$, taking the difference in the observables with respect to the nominal procedure as the uncertainty estimate. The uncertainty due to the finite Monte Carlo sample size was found to be negligible since fewer bins were used in the unfolding than for the templates. These sources of uncertainty are labelled "method-specific uncertainties" in table 3.

\subsubsection{Detector modelling}

The mis-modelling of lepton trigger, reconstruction and selection efficiencies in simulation was corrected for by scale factors derived from auxiliary measurements of the processes $Z \rightarrow \mu \mu$ and $Z \rightarrow e e[50,51]$. The uncertainties were evaluated by changing the event selection of the supplementary measurement and by testing the stability of the results against changing LHC and ATLAS run conditions. The same processes were used to measure the lepton momentum scale and resolution. Scale factors and their uncertainties were derived to match the simulation to observed distributions. Details are given in ref. [39].

The jet energy scale was derived using information from test-beam data, LHC collision data and simulation. Its uncertainty varies between $2.5 \%$ and $7 \%$ in the central $\eta$ region, depending on jet $p_{\mathrm{T}}$ and $\eta$ [52]. This includes uncertainties in the flavour composition of the samples and mis-measurements from close-by jets. An additional $p_{\mathrm{T}}$-dependent uncertainty of up to $2.5 \%$ was assigned to jets matched to $b$-quarks (using Monte Carlo generator-level information) due to differences between light-quark and gluon jets as opposed to jets containing $b$-hadrons. Additional uncertainties of up to 5\% (8\%) in the central (forward) region were added due to pileup. The energy resolution for jets in Monte Carlo simulation was adjusted to that observed in data. Uncertainties on the energy resolution of 4-45\%, decreasing with jet $p_{\mathrm{T}}$, were assigned. The reconstruction efficiency of jets was found to be in good agreement with the predictions from simulation and uncertainties of $1-2 \%$ were assigned. 


\begin{tabular}{|llll|}
\hline Source & \multicolumn{3}{l}{ Uncertainties } \\
& $F_{0}$ & $F_{\mathrm{L}}$ & $F_{\mathrm{R}}$ \\
\hline Signal and background modelling & & \\
\hline Generator choice & 0.012 & 0.009 & 0.004 \\
ISR/FSR & 0.015 & 0.008 & 0.007 \\
PDF & 0.011 & 0.006 & 0.006 \\
Top quark mass & 0.016 & 0.009 & 0.008 \\
Misidentified leptons & 0.020 & 0.013 & 0.007 \\
W+jets & 0.016 & 0.008 & 0.008 \\
Other backgrounds & 0.006 & 0.003 & 0.003 \\
Method-specific uncertainties & 0.031 & 0.016 & 0.035 \\
\hline Detector modelling & & & \\
\hline Lepton reconstruction & 0.013 & 0.006 & 0.007 \\
Jet energy scale & 0.026 & 0.014 & 0.012 \\
Jet reconstruction & 0.012 & 0.005 & 0.007 \\
b-tagging & 0.007 & 0.003 & 0.004 \\
Calorimeter readout & 0.009 & 0.005 & 0.004 \\
Luminosity and pileup & 0.009 & 0.004 & 0.005 \\
\hline Total systematic uncertainty & 0.06 & 0.03 & 0.04 \\
\hline
\end{tabular}

Table 3. Sources of systematic uncertainty and their impact on the measured $W$ boson helicity fractions for the combined single-lepton and dilepton channels. The systematic uncertainties were symmetrized by using the larger uncertainty.

The uncertainties on the momenta of electrons, muons and jets were propagated into the missing transverse momentum. A $10 \%$ uncertainty from pileup was added in addition.

The $b$-tagging efficiencies and mis-tag rates have been measured in data [42]. Jet $p_{\mathrm{T}^{-}}$ dependent scale factors, applied to simulation to match the data, have uncertainties which range from $9 \%$ to $16 \%$ and $12 \%$ to $45 \%$, respectively.

The uncertainty on the measured luminosity was estimated to be $3.7 \%$ [15].

Due to a hardware failure, a small, rectangular region of the ATLAS electromagnetic calorimeter could not be read out in a subset of the data $\left(0.87 \mathrm{fb}^{-1}\right)$. Data and Monte Carlo events in which a jet or an electron were close to the affected calorimeter region were rejected. The systematic uncertainty labelled "calorimeter readout" in table 3 was evaluated by varying the criteria to reject these events. 

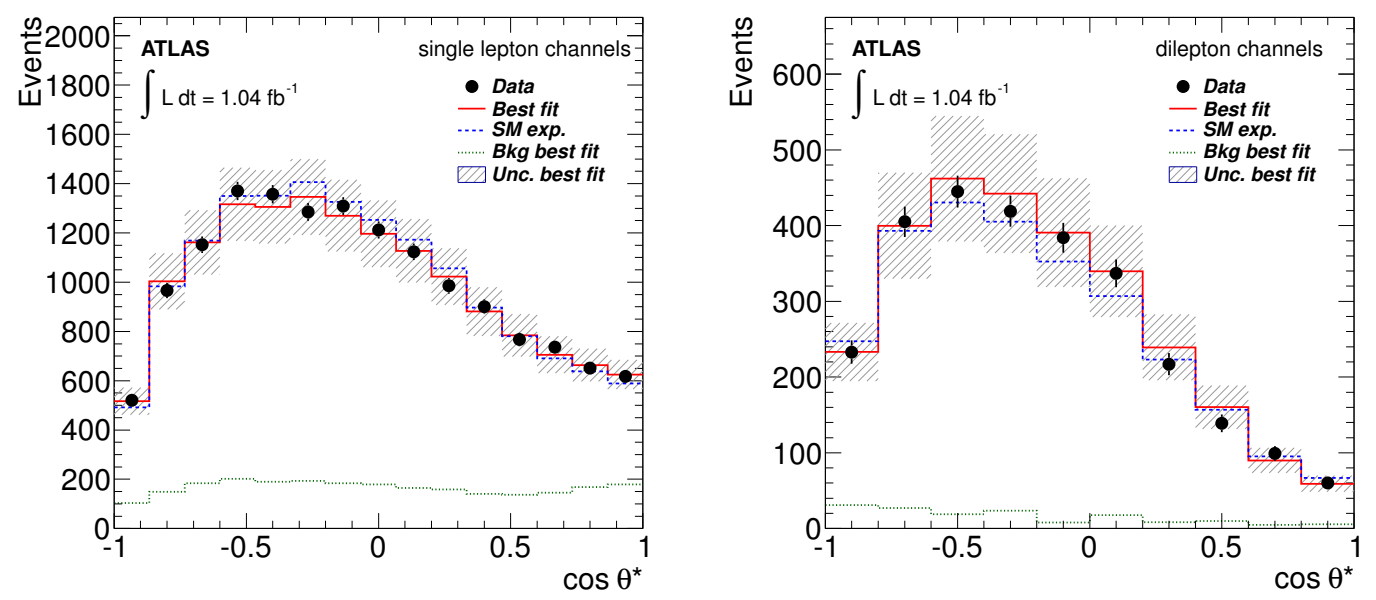

Figure 4. Distributions of the reconstructed $\cos \theta^{*}$ used in the template method for data (markers), fitted background (dotted line), the Standard Model prediction (dashed line) and the best fit value (solid line) for the (left) single-lepton and (right) dilepton channels. The total uncertainties on the helicity fractions for the best fit values are represented by the grey band. For the dilepton channels, each event contributed with two entries, corresponding to the two leptonic decays of the $W$ bosons.

\section{Results}

The two methods discussed in section 4 were applied to the data set described in section 2 . Figure 4 shows the observed distribution of $\cos \theta^{*}$ in the single-lepton and dilepton channels together with the sum of the templates scaled to the best fit parameters obtained from the template method as well as the Standard Model expectations. Figure 5 shows the distribution of $\cos \theta^{*}$ in the single-lepton and dilepton channels after the background subtraction and the correction for detector and reconstruction effects as used by the asymmetry method as well as the Standard Model expectations.

The combination of the individual measurements of the $W$ boson helicity fractions $\left(F_{0}\right.$ and $F_{\mathrm{L}}$ ) and asymmetries $\left(A_{+}\right.$and $\left.A_{-}\right)$in the single-lepton and dilepton channels was done using the best linear unbiased estimator (BLUE) method [53, 54]. The impact of the systematic uncertainties described in section 5 was studied and the results are summarized in table 4, which also gives the combination of all channels for each method. The results are compatible with each other and with the final combination. In addition, the template fit was repeated with $F_{\mathrm{R}}$ fixed to zero. ${ }^{3}$ With the precision of the current measurements, this differs negligibly from the Standard Model value, and also follows the approach suggested in ref. [13]. The results can also be found in table 4 .

The results for the angular asymmetries from the single-lepton channels are $A_{+}=$ $0.52 \pm 0.02$ (stat.) \pm 0.03 (syst.) and $A_{-}=-0.84 \pm 0.01$ (stat.) \pm 0.02 (syst.) whereas

\footnotetext{
${ }^{3}$ In the evaluation of the angular asymmetries no assumption is made for the helicity fractions, so it is not possible to fix $F_{\mathrm{R}}$ to zero.
} 

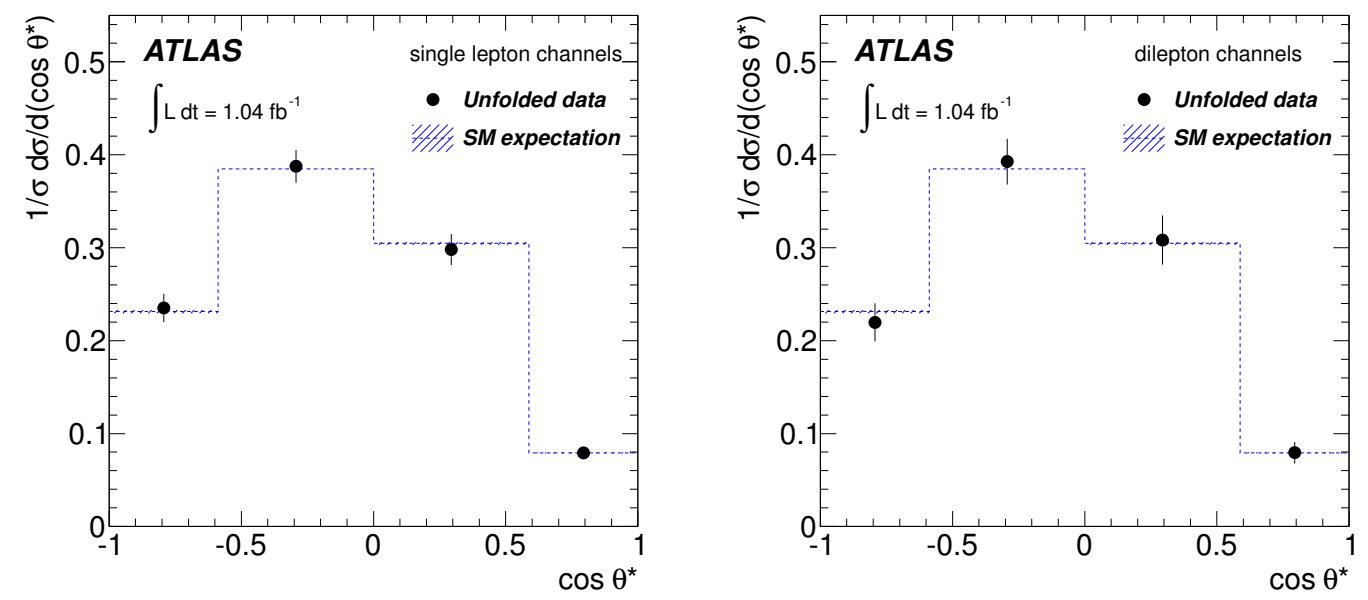

Figure 5. Unfolded distributions of $\cos \theta^{*}$ for the (left) single-lepton and (right) dilepton channels. The error bars on unfolded data (markers) include both the statistical and systematic contributions. For comparison, the Standard Model NNLO QCD prediction (dashed line) and its uncertainty [4] are also shown.

the results for the dilepton channels are $A_{+}=0.56 \pm 0.02$ (stat.) \pm 0.04 (syst.) and $A_{-}=-0.84 \pm 0.02$ (stat.) \pm 0.04 (syst.).

Most of the measurements and the combined result are limited by systematic uncertainties. The largest sources of uncertainty are the signal and background modelling, as well as the jet energy scale and jet reconstruction. The template fits are more sensitive to shape-related uncertainties, such as ISR/FSR and jet reconstruction, while the angular asymmetries are more sensitive to background normalization uncertainties, due to the background subtraction which needs to be performed.

\subsection{Combination}

The results presented in the previous sections were combined using the BLUE method. Both the statistical correlations between analyses, and the correlations of systematic uncertainties, were taken into account. The statistical correlations were estimated to be between $40 \%$ and $49 \%$ for the single-lepton channels and between $83 \%$ and $89 \%$ for the dilepton channels using pseudo-experiments obtained from simulated samples. The backgroundrelated systematic uncertainties were assumed to be fully correlated within single-lepton channels and within dilepton channels, but uncorrelated between single-lepton and dilepton measurements. The only exception is the uncertainty due to misidentified lepton background which depends on the lepton flavour and thus belongs to the group of lepton-related uncertainties, which were assumed to be fully correlated between the channels with same flavour leptons. The method systematic uncertainties were assumed to be uncorrelated between channels and the remaining sources of systematic uncertainty were assumed to be fully correlated between channels. Various tests were performed in which the correlations among the sources of systematic uncertainty were varied. It was found that the values 


\begin{tabular}{|lccr|}
\hline Channel & $F_{0}$ & $F_{\mathrm{L}}$ & $F_{\mathrm{R}}$ \\
\hline$W$ boson helicity fractions from the template fit & \\
\hline Single leptons & $0.57 \pm 0.06 \pm 0.09$ & $0.37 \pm 0.03 \pm 0.04$ & $0.07 \pm 0.03 \pm 0.06$ \\
Dileptons & $0.92 \pm 0.10 \pm 0.10$ & $0.17 \pm 0.06 \pm 0.07$ & $-0.09 \pm 0.05 \pm 0.06$ \\
Combination & $0.66 \pm 0.06 \pm 0.07$ & $0.33 \pm 0.03 \pm 0.03$ & $0.01 \pm 0.03 \pm 0.06$ \\
\hline$F_{\mathrm{R}}$ fixed & $0.66 \pm 0.03 \pm 0.04$ & $0.34 \pm 0.03 \pm 0.04$ & 0 (fixed) \\
\hline$W$ boson helicity fractions from the angular asymmetries & \\
\hline Single leptons & $0.66 \pm 0.03 \pm 0.08$ & $0.33 \pm 0.02 \pm 0.05$ & $0.01 \pm 0.01 \pm 0.04$ \\
Dileptons & $0.74 \pm 0.06 \pm 0.10$ & $0.27 \pm 0.03 \pm 0.05$ & $-0.01 \pm 0.03 \pm 0.05$ \\
Combination & $0.67 \pm 0.04 \pm 0.07$ & $0.32 \pm 0.02 \pm 0.04$ & $0.01 \pm 0.02 \pm 0.04$ \\
\hline Overall combination & $0.67 \pm 0.03 \pm 0.06$ & $0.32 \pm 0.02 \pm 0.03$ & $0.01 \pm 0.01 \pm 0.04$ \\
\hline
\end{tabular}

Table 4. Summary of the $W$ boson helicity fractions measured using the two different techniques described and the combination. The quoted uncertainties are the statistical (first) and the systematic (second) uncertainties.

assumed for the correlations were conservative. The systematic uncertainties on the combined values are summarized in table 3 . The four measurements of the helicity fractions and the combined values are shown in table 4 and figure 6.

The individual measurements agree reasonably well within their total uncertainties. The $\chi^{2} / d o f$ for the global combination of the template fit and asymmetries measurements was 0.8 with a $\chi^{2}$-probability of $75 \%$, where $d o f$ is the number of degrees of freedom. The largest difference between two measurements is that between the single-lepton and dilepton channels obtained with the template method. Since the measurements were performed in five independent channels (single electron, single muon, ee, $e \mu$ and $\mu \mu$ ), the combination was performed based on the five individual measurements taking into account all correlations. The $\chi^{2} /$ dof calculated using the BLUE method for this combination was 1.3 with a $\chi^{2}$-probability of $23 \%$.

The combined $W$ boson helicity fractions are:

$$
\begin{aligned}
& F_{0}=0.67 \pm 0.03 \text { (stat.) } \pm 0.06 \text { (syst.), } \\
& F_{\mathrm{L}}=0.32 \pm 0.02 \text { (stat.) } \pm 0.03 \text { (syst.), } \\
& F_{\mathrm{R}}=0.01 \pm 0.01 \text { (stat.) } \pm 0.04 \text { (syst.) }
\end{aligned}
$$

The correlation coefficient between $F_{0}$ and $F_{\mathrm{L}}$ was estimated to be -0.96 . For completeness, these results can be translated into angular asymmetries, yielding $A_{+}=0.53 \pm 0.02$ and $A_{-}=-0.84 \pm 0.02$.

An alternative analysis, based on requiring two $b$-tagged jets to further suppress the $W+$ jets background and events with misidentified leptons, was used to measure the helicity fractions in the single-lepton channels as a cross-check. The observed $\cos \theta^{*}$ distributions were corrected by subtracting the expected background contributions and were unfolded 


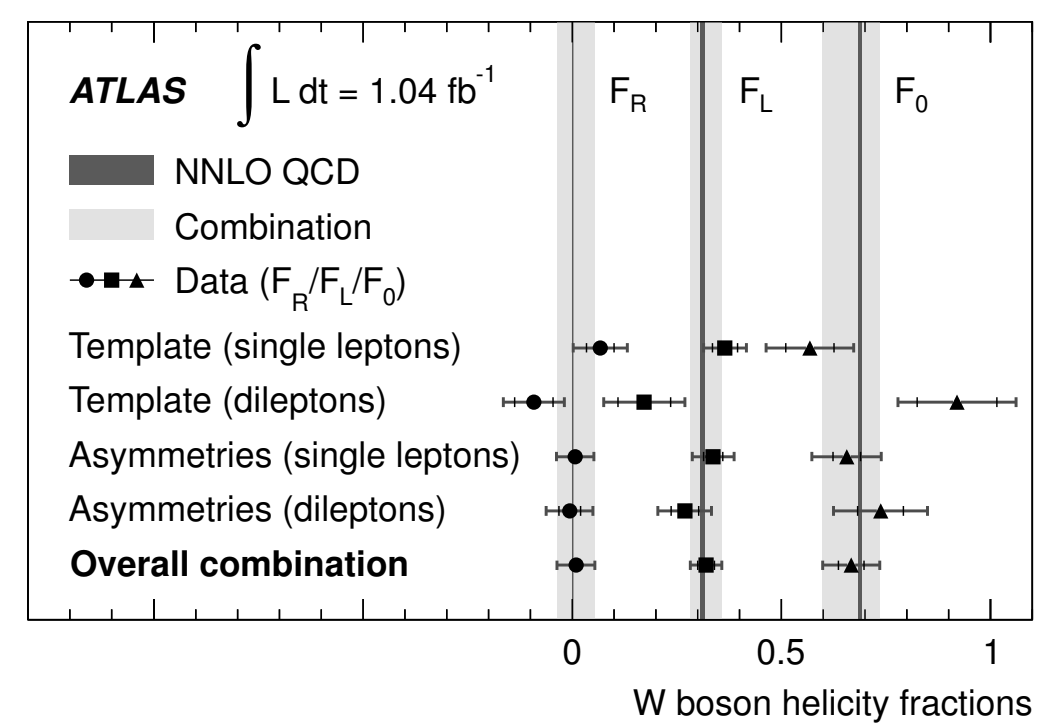

Figure 6. Overview of the four measurements of the $W$ boson helicity fractions and the combined values. The error bars correspond to the statistical and total uncertainties.

using correction functions in an iterative method similar to the one used in the measurement of the angular asymmetries described in section 4.2. The results were found to be in agreement with the single-lepton measurements presented in this paper.

\subsection{Constraints on the $W t b$ vertex structure}

Any deviation of $F_{0}, F_{\mathrm{L}}, F_{\mathrm{R}}$ (or $A_{+}$and $A_{-}$) from the Standard Model prediction could be caused by new physics contributing to the $W t b$ vertex. Such new interactions associated with the top quark may exist at higher energies. New physics can be parameterized in terms of an effective Lagrangian [11] above the electroweak symmetry breaking scale of $v=246 \mathrm{GeV}$. After electroweak symmetry breaking, the $W t b$ Lagrangian $[12,55]$ is:

$$
\mathcal{L}_{W t b}=-\frac{g}{\sqrt{2}} \bar{b} \gamma^{\mu}\left(V_{\mathrm{L}} P_{\mathrm{L}}+V_{\mathrm{R}} P_{\mathrm{R}}\right) t W_{\mu}^{-}-\frac{g}{\sqrt{2}} \bar{b} \frac{i \sigma^{\mu \nu} q_{\nu}}{M_{W}}\left(g_{\mathrm{L}} P_{\mathrm{L}}+g_{\mathrm{R}} P_{\mathrm{R}}\right) t W_{\mu}^{-}+\text {h.c. }
$$

where $P_{\mathrm{L}}\left(P_{\mathrm{R}}\right)$ is the left-handed (right-handed) chirality operator and

$$
V_{\mathrm{L}}=V_{t b}+C_{\phi q}^{(3,3+3)} \frac{v^{2}}{\Lambda^{2}}, \quad V_{\mathrm{R}}=\frac{1}{2} C_{\phi \phi}^{33 *} \frac{v^{2}}{\Lambda^{2}}, \quad g_{\mathrm{L}}=\sqrt{2} C_{d W}^{33 *} \frac{v^{2}}{\Lambda^{2}}, \quad g_{\mathrm{R}}=\sqrt{2} C_{u W}^{33} \frac{v^{2}}{\Lambda^{2}} .
$$

The parameter $\Lambda$ is the new physics scale and $C_{\phi q}^{(3,3+3)}, C_{\phi \phi}^{33 *}, C_{d W}^{33 *}$ and $C_{u W}^{33}$ are the effective operator coefficients $[13,55]$. The anomalous couplings $V_{\mathrm{R}}, g_{\mathrm{L}}, g_{\mathrm{R}}$, generated by dimension-six operators, are absent in the Standard Model at tree level, while the coupling $V_{t b}$ receives a correction from the operator $O_{\phi q}^{(3,3+3)}$.

Limits on anomalous couplings $\left(V_{\mathrm{R}}, g_{\mathrm{L}}\right.$ and $\left.g_{\mathrm{R}}\right)$ were obtained from the combined measurement of the $W$ boson helicity fractions by exploiting their dependence on these 


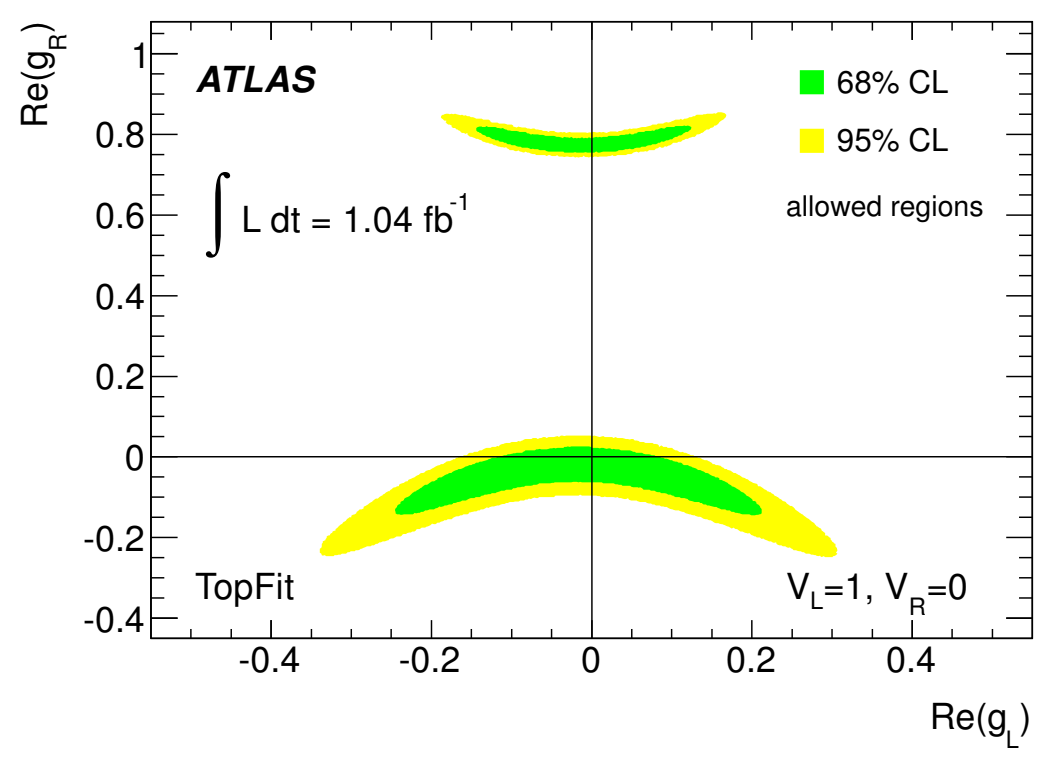

Figure 7. Allowed regions at $68 \%$ and $95 \%$ confidence level (CL) for the $W t b$ anomalous couplings $g_{\mathrm{L}}$ and $g_{\mathrm{R}}$. In the Standard Model, the anomalous couplings vanish at tree level [59].

couplings, as implemented in the TopFit program $[10,56]$ and normalizing to $V_{\mathrm{L}}=1$. The allowed regions of $\left(g_{\mathrm{L}}, g_{\mathrm{R}}\right)$ are shown in figure 7 , assuming $V_{\mathrm{R}}=0$. The upper disconnected region in the plot shows a large- $g_{\mathrm{R}}$ second solution to the quadratic equation relating the observables to the anomalous couplings. However, this region is disfavored by the measured cross-section for single top production at the Tevatron $[17,57,58]$.

In addition to this two-dimensional limit it is useful to set limits on single anomalous couplings, taking only one of them non-zero at a time. These are, at $95 \%$ confidence level,

$$
\begin{aligned}
& \operatorname{Re}\left(V_{\mathrm{R}}\right) \in[-0.20,0.23] \rightarrow \frac{\operatorname{Re}\left(C_{\phi \phi}^{33}\right)}{\Lambda^{2}} \in[-6.7,7.8] \mathrm{TeV}^{-2}, \\
& \operatorname{Re}\left(g_{\mathrm{L}}\right) \in[-0.14,0.11] \rightarrow \frac{\operatorname{Re}\left(C_{d W}^{33}\right)}{\Lambda^{2}} \in[-1.6,1.2] \mathrm{TeV}^{-2}, \\
& \operatorname{Re}\left(g_{\mathrm{R}}\right) \in[-0.08,0.04] \rightarrow \frac{\operatorname{Re}\left(C_{u W}^{33}\right)}{\Lambda^{2}} \in[-1.0,0.5] \mathrm{TeV}^{-2} .
\end{aligned}
$$

The considered $W$ boson helicity observables also allow a second region for $g_{\mathrm{R}}$ when the remaining anomalous couplings vanish: $\operatorname{Re}\left(g_{\mathrm{R}}\right) \in[0.75,0.80]$ at $95 \%$ confidence level. It should be noticed, however, that such large coupling values would imply a single top production cross-section value disfavored by the Tevatron measurements $[17,57,58]$. Using a Bayesian approach [60], the measurement of the $W$ boson helicity fractions with $F_{\mathrm{R}}$ fixed at zero, was translated into a $95 \%$ probability interval on $\operatorname{Re}\left(C_{u W}^{33}\right) / \Lambda^{2}$, as proposed in ref. [13]. This interval was found to be $[-0.9,2.3] \mathrm{TeV}^{-2}$.

It can be seen that the limits on $C_{d W}^{33}$ (mediating the production of right-handed $b$ quarks in the top decay) are of the same order of magnitude as the limits on $C_{u W}^{33}$ (involving 
left-handed quarks). This reflects a good sensitivity to the effective operator corresponding to $C_{d W}^{33}$, even if its contribution is suppressed by $1 / \Lambda^{2}$ instead of $1 / \Lambda$ [61].

These limits are more stringent than those obtained by the D $\varnothing$ Collaboration [58, 62]. ${ }^{4}$ Indirect, model-dependent limits on the anomalous couplings have been inferred from measurements of radiative $B$-meson decays, measurements of $B \bar{B}$-mixing and electroweak precision data [63-67], but these limits include assumptions on the absence of additional new physics effects that are not needed in this analysis.

\section{Conclusions}

A measurement of the polarization of the $W$ bosons in top quark decays was presented, based on $1.04 \mathrm{fb}^{-1}$ of data collected with the ATLAS detector in 2011. The single-lepton and dilepton decay topologies of top quark pairs were considered in the analysis.

The helicity fractions obtained from a combination of template fits to the reconstructed $\cos \theta^{*}$ distributions and angular asymmetries calculated from the unfolded $\cos \theta^{*}$ distributions are $F_{0}=0.67 \pm 0.07, F_{\mathrm{L}}=0.32 \pm 0.04$ and $F_{\mathrm{R}}=0.01 \pm 0.05$. These results are in agreement with NNLO QCD predictions and are more precise than previous results obtained by the CDF and DØ Collaborations [6-8].

Limits on the $W t b$ vertex anomalous couplings were obtained from the combined results on the $W$ boson helicity fractions. These results are consistent with the $(V-A)$ structure of the $W t b$ vertex and improve on the previously obtained limits [58].

\section{Acknowledgments}

We thank CERN for the very successful operation of the LHC, as well as the support staff from our institutions without whom ATLAS could not be operated efficiently.

We acknowledge the support of ANPCyT, Argentina; YerPhI, Armenia; ARC, Australia; BMWF, Austria; ANAS, Azerbaijan; SSTC, Belarus; CNPq and FAPESP, Brazil; NSERC, NRC and CFI, Canada; CERN; CONICYT, Chile; CAS, MOST and NSFC, China; COLCIENCIAS, Colombia; MSMT CR, MPO CR and VSC CR, Czech Republic; DNRF, DNSRC and Lundbeck Foundation, Denmark; EPLANET and ERC, European Union; IN2P3-CNRS, CEA-DSM/IRFU, France; GNAS, Georgia; BMBF, DFG, HGF, MPG and AvH Foundation, Germany; GSRT, Greece; ISF, MINERVA, GIF, DIP and Benoziyo Center, Israel; INFN, Italy; MEXT and JSPS, Japan; CNRST, Morocco; FOM and NWO, Netherlands; RCN, Norway; MNiSW, Poland; GRICES and FCT, Portugal; MERYS (MECTS), Romania; MES of Russia and ROSATOM, Russian Federation; JINR; MSTD, Serbia; MSSR, Slovakia; ARRS and MVZT, Slovenia; DST/NRF, South Africa; MICINN, Spain; SRC and Wallenberg Foundation, Sweden; SER, SNSF and Cantons of Bern and Geneva, Switzerland; NSC, Taiwan; TAEK, Turkey; STFC, the Royal Society and Leverhulme Trust, United Kingdom; DOE and NSF, United States of America.

The crucial computing support from all WLCG partners is acknowledged gratefully, in particular from CERN and the ATLAS Tier-1 facilities at TRIUMF (Canada), NDGF

\footnotetext{
${ }^{4}$ The limits from the $\mathrm{D} \varnothing$ Collaboration were derived assuming a massless $b$-quark.
} 
(Denmark, Norway, Sweden), CC-IN2P3 (France), KIT/GridKA (Germany), INFN-CNAF (Italy), NL-T1 (Netherlands), PIC (Spain), ASGC (Taiwan), RAL (U.K.) and BNL (U.S.A.) and in the Tier-2 facilities worldwide.

Open Access. This article is distributed under the terms of the Creative Commons Attribution License which permits any use, distribution and reproduction in any medium, provided the original author(s) and source are credited.

\section{References}

[1] CDF collaboration, F. Abe et al., Observation of top quark production in p p p collisions, Phys. Rev. Lett. 74 (1995) 2626 [hep-ex/9503002] [INSPIRE].

[2] DØ collaboration, S. Abachi et al., Observation of the top quark, Phys. Rev. Lett. 74 (1995) 2632 [hep-ex/9503003] [INSPIRE].

[3] Tevatron Electroweak Working Group, CDF and D $\varnothing$ collaborations, Combination of $C D F$ and $D \varnothing$ results on the mass of the top quark using up to $5.8 \mathrm{fb}^{-1}$ of data, arXiv:1107.5255 [INSPIRE].

[4] A. Czarnecki, J.G. Korner and J.H. Piclum, Helicity fractions of $W$ bosons from top quark decays at NNLO in QCD, Phys. Rev. D 81 (2010) 111503 [arXiv:1005.2625] [InSPIRE].

[5] G.L. Kane, G. Ladinsky and C. Yuan, Using the top quark for testing Standard Model polarization and CP predictions, Phys. Rev. D 45 (1992) 124 [INSPIRE].

[6] CDF and D $\varnothing$ collaborations, T. Aaltonen et al., Combination of CDF and D measurements of the $W$ boson helicity in top quark decays, arXiv:1202.5272 [INSPIRE].

[7] CDF collaboration, T. Aaltonen et al., Measurement of $W$-boson polarization in top-quark decay in p $\bar{p}$ collisions at $\sqrt{s}=1.96 \mathrm{TeV}$, Phys. Rev. Lett. 105 (2010) 042002 [arXiv: 1003.0224] [INSPIRE].

[8] DØ collaboration, V.M. Abazov et al., Measurement of the $W$ boson helicity in top quark decays using $5.4 \mathrm{fb}^{-1}$ of $p \bar{p}$ collision data, Phys. Rev. D 83 (2011) 032009 [arXiv: 1011.6549] [INSPIRE].

[9] F. del Aguila and J. Aguilar-Saavedra, Precise determination of the Wtb couplings at CERN LHC, Phys. Rev. D 67 (2003) 014009 [hep-ph/0208171] [INSPIRE].

[10] J. Aguilar-Saavedra, J. Carvalho, N.F. Castro, F. Veloso and A. Onofre, Probing anomalous Wtb couplings in top pair decays, Eur. Phys. J. C 50 (2007) 519 [hep-ph/0605190] [INSPIRE].

[11] W. Buchmüller and D. Wyler, Effective Lagrangian analysis of new interactions and flavor conservation, Nucl. Phys. B 268 (1986) 621 [INSPIRE].

[12] J. Aguilar-Saavedra, A minimal set of top anomalous couplings, Nucl. Phys. B 812 (2009) 181 [arXiv:0811.3842] [INSPIRE].

[13] C. Zhang and S. Willenbrock, Effective-field-theory approach to top-quark production and decay, Phys. Rev. D 83 (2011) 034006 [arXiv: 1008.3869] [inSPIRE].

[14] ATLAS collaboration, G. Aad et al., The ATLAS experiment at the CERN Large Hadron Collider, 2008 JINST 3 S08003 [INSPIRE]. 
[15] ATLAS collaboration, Luminosity determination in pp collisions at $\sqrt{s}=7 \mathrm{TeV}$ using the ATLAS detector in 2011, ATLAS-CONF-2011-116, CERN, Geneva Switzerland (2011).

[16] ATLAS collaboration, G. Aad et al., Luminosity determination in pp collisions at $\sqrt{s}=7$ TeV using the ATLAS detector at the LHC, Eur. Phys. J. C 71 (2011) 1630 [arXiv:1101.2185] [INSPIRE].

[17] J. Aguilar-Saavedra, Single top quark production at LHC with anomalous Wtb couplings, Nucl. Phys. B 804 (2008) 160 [arXiv:0803.3810] [InSPIRE].

[18] J. Pumplin et al., New generation of parton distributions with uncertainties from global QCD analysis, JHEP 07 (2002) 012 [hep-ph/0201195] [INSPIRE].

[19] T. Sjöstrand, S. Mrenna and P.Z. Skands, PYTHIA 6.4 physics and manual, JHEP 05 (2006) 026 [hep-ph/0603175] [INSPIRE].

[20] S. Frixione and B.R. Webber, Matching NLO QCD computations and parton shower simulations, JHEP 06 (2002) 029 [hep-ph/0204244] [INSPIRE].

[21] S. Frixione, P. Nason and B.R. Webber, Matching NLO QCD and parton showers in heavy flavor production, JHEP 08 (2003) 007 [hep-ph/0305252] [INSPIRE].

[22] S. Frixione, E. Laenen, P. Motylinski and B.R. Webber, Single-top production in MC@NLO, JHEP 03 (2006) 092 [hep-ph/0512250] [INSPIRE].

[23] P.M. Nadolsky et al., Implications of CTEQ global analysis for collider observables, Phys. Rev. D 78 (2008) 013004 [arXiv:0802.0007] [INSPIRE].

[24] M.L. Mangano, M. Moretti, F. Piccinini, R. Pittau and A.D. Polosa, ALPGEN, a generator for hard multiparton processes in hadronic collisions, JHEP 07 (2003) 001 [hep-ph/0206293] [INSPIRE].

[25] G. Corcella et al., HERWIG 6: an event generator for hadron emission reactions with interfering gluons (including supersymmetric processes), JHEP 01 (2001) 010 [hep-ph/0011363] [INSPIRE].

[26] S. Frixione, E. Laenen, P. Motylinski, B.R. Webber and C.D. White, Single-top hadroproduction in association with a $W$ boson, JHEP 07 (2008) 029 [arXiv:0805.3067] [INSPIRE].

[27] T. Gleisberg et al., Event generation with SHERPA 1.1, JHEP 02 (2009) 007 [arXiv:0811.4622] [INSPIRE].

[28] J. Butterworth, J.R. Forshaw and M. Seymour, Multiparton interactions in photoproduction at HERA, Z. Phys. C 72 (1996) 637 [hep-ph/9601371] [INSPIRE].

[29] ATLAS collaboration, New ATLAS event generator tunes to 2010 data, ATL-PHYS-PUB-2011-008, CERN, Geneva Switzerland (2011).

[30] B.P. Kersevan and E. Richter-Was, The Monte Carlo event generator AcerMC version 2.0 with interfaces to PYTHIA 6.2, HERWIG 6.5 and ARIADNE 4.1, hep-ph/0405247 [INSPIRE].

[31] P. Nason, A new method for combining NLO QCD with shower Monte Carlo algorithms, JHEP 11 (2004) 040 [hep-ph/0409146] [INSPIRE].

[32] S. Frixione, P. Nason and C. Oleari, Matching NLO QCD computations with parton shower simulations: the POWHEG method, JHEP 11 (2007) 070 [arXiv:0709.2092] [INSPIRE]. 
[33] GEANT4 collaboration, S. Agostinelli et al., GEANT4: a simulation toolkit, Nucl. Instrum. Meth. A 506 (2003) 250 [inSPIRE].

[34] ATLAS collaboration, G. Aad et al., The ATLAS simulation infrastructure, Eur. Phys. J. C 70 (2010) 823 [arXiv:1005.4568] [InSPIRE].

[35] S. Moch and P. Uwer, Theoretical status and prospects for top-quark pair production at hadron colliders, Phys. Rev. D 78 (2008) 034003 [arXiv:0804.1476] [InSPIRE].

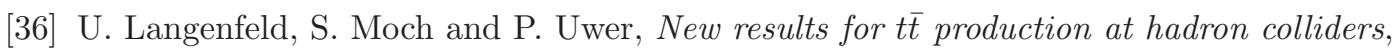
arXiv:0907 .2527 [INSPIRE].

[37] M. Aliev et al., HATHOR: HAdronic Top and Heavy quarks crOss section calculatoR, Comput. Phys. Commun. 182 (2011) 1034 [arXiv:1007.1327] [INSPIRE].

[38] ATLAS collaboration, G. Aad et al., Measurement of the cross section for top-quark pair production in $p p$ collisions at $\sqrt{s}=7$ TeV with the ATLAS detector using final states with

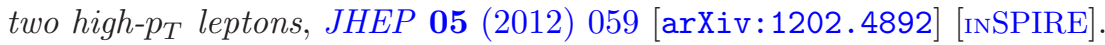

[39] ATLAS collaboration, G. Aad et al., Measurement of the top quark-pair production cross section with ATLAS in pp collisions at $\sqrt{s}=7$ TeV, Eur. Phys. J. C 71 (2011) 1577 [arXiv: 1012.1792] [INSPIRE].

[40] ATLAS collaboration, G. Aad et al., Measurement of the charge asymmetry in top quark pair production in pp collisions at $\sqrt{s}=7$ TeV using the ATLAS detector, arXiv:1203.4211 [INSPIRE].

[41] M. Cacciari, G.P. Salam and G. Soyez, The anti- $k_{t}$ jet clustering algorithm, JHEP 04 (2008) 063 [arXiv:0802.1189] [INSPIRE].

[42] ATLAS collaboration, Measurement of the rate of collisions from satellite bunches for the april-may 2010 LHC luminosity calibration, ATLAS-CONF-2010-102, CERN, Geneva Switzerland (2010).

[43] ATLAS collaboration, G. Aad et al., Measurement of the top quark mass with the template method in the $t \bar{t} \rightarrow$ lepton + jets channel using ATLAS data, Eur. Phys. J. C (2012) [arXiv:1203.5755] [INSPIRE].

[44] ATLAS collaboration, Expected performance of the ATLAS experiment: detector, trigger and physics, CERN-OPEN-2008-020, CERN, Geneva Switzerland (2009).

[45] P.Z. Skands, Tuning Monte Carlo generators: the Perugia tunes, Phys. Rev. D 82 (2010) 074018 [arXiv: 1005.3457] [INSPIRE].

[46] TeV4LHC QCD Working Group collaboration, M.G. Albrow et al., Tevatron-for-LHC report of the QCD working group, hep-ph/0610012 [INSPIRE].

[47] A. Buckley, H. Hoeth, H. Lacker, H. Schulz and J.E. von Seggern, Systematic event generator tuning for the LHC, Eur. Phys. J. C 65 (2010) 331 [arXiv:0907.2973] [INSPIRE].

[48] F.A. Berends, H. Kuijf, B. Tausk and W. Giele, On the production of a $W$ and jets at hadron colliders, Nucl. Phys. B 357 (1991) 32 [InSPIRE].

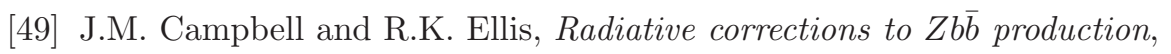
Phys. Rev. D 62 (2000) 114012 [hep-ph/0006304] [INSPIRE].

[50] ATLAS collaboration, Muon momentum resolution in first pass reconstruction of $p p$ collision data recorded by ATLAS in 2010, ATLAS-CONF-2011-046, CERN, Geneva Switzerland (2011). 
[51] ATLAS collaboration, G. Aad et al., Electron performance measurements with the ATLAS detector using the 2010 LHC proton-proton collision data, Eur. Phys. J. C 72 (2012) 1909 [arXiv:1110.3174] [INSPIRE].

[52] ATLAS collaboration, G. Aad et al., Jet energy measurement with the ATLAS detector in proton-proton collisions at $\sqrt{s}=7 \mathrm{TeV}$, arXiv:1112.6426 [INSPIRE].

[53] L. Lyons, D. Gibaut and P. Clifford, How to combine correlated estimates of a single physical quantity, Nucl. Instrum. Meth. A 270 (1988) 110 [INSPIRE].

[54] A. Valassi, Combining correlated measurements of several different physical quantities, Nucl. Instrum. Meth. A 500 (2003) 391 [inSPIRE].

[55] J. Aguilar-Saavedra, A minimal set of top-Higgs anomalous couplings, Nucl. Phys. B 821 (2009) 215 [arXiv:0904.2387] [INSPIRE].

[56] J. Aguilar-Saavedra and J. Bernabeu, $W$ polarisation beyond helicity fractions in top quark decays, Nucl. Phys. B 840 (2010) 349 [arXiv: 1005.5382] [INSPIRE].

[57] CDF and DØ collaborations, T.E.W. Group, Combination of CDF and DØ measurements of the single top production cross section, arXiv:0908.2171 [INSPIRE].

[58] DØ collaboration, V.M. Abazov et al., Combination of searches for anomalous top quark couplings with $5.4 \mathrm{fb}^{-1}$ of $p \bar{p}$ collisions, arXiv:1204.2332 [INSPIRE].

[59] H. Do, S. Groote, J. Korner and M. Mauser, Electroweak and finite width corrections to top quark decays into transverse and longitudinal $W$ bosons, Phys. Rev. D 67 (2003) 091501 [hep-ph/0209185] [INSPIRE].

[60] A. Caldwell, D. Kollar and K. Kroninger, BAT: the Bayesian Analysis Toolkit, Comput. Phys. Commun. 180 (2009) 2197 [arXiv: 0808. 2552] [INSPIRE].

[61] J.A. Aguilar-Saavedra, Effective operators in top physics, PoS(ICHEP 2010) 378 [arXiv: 1008.3225] [INSPIRE].

[62] DØ collaboration, V.M. Abazov et al., Search for anomalous Wtb couplings in single top quark production in p $\bar{p}$ collisions at $\sqrt{s}=1.96 \mathrm{TeV}$, Phys. Lett. B $\mathbf{7 0 8}$ (2012) 21 [arXiv: 1110.4592] [INSPIRE].

[63] B. Grzadkowski and M. Misiak, Anomalous Wtb coupling effects in the weak radiative B-meson decay, Phys. Rev. D 78 (2008) 077501 [Erratum ibid. D 84 (2011) 059903] [arXiv: 0802.1413] [INSPIRE].

[64] F. del Aguila et al., Collider aspects of flavour physics at high Q, Eur. Phys. J. C 57 (2008) 183 [arXiv:0801.1800] [inSPIRE].

[65] J. Drobnak, S. Fajfer and J.F. Kamenik, Probing anomalous tWb interactions with rare $B$ decays, Nucl. Phys. B 855 (2012) 82 [arXiv:1109.2357] [INSPIRE].

[66] J. Drobnak, S. Fajfer and J.F. Kamenik, Interplay of $t \rightarrow b W$ decay and $B_{q}$ meson mixing in Minimal Flavor Violating models, Phys. Lett. B 701 (2011) 234 [arXiv:1102.4347] [INSPIRE].

[67] C. Zhang, N. Greiner and S. Willenbrock, Constraints on non-standard top quark couplings, arXiv: 1201.6670 [INSPIRE]. 


\section{The ATLAS collaboration}

G. Aad ${ }^{48}$, B. Abbott ${ }^{111}$, J. Abdallah ${ }^{11}$, S. Abdel Khalek ${ }^{115}$, A.A. Abdelalim ${ }^{49}$, O. Abdinov ${ }^{10}$, B. Abi ${ }^{112}$, M. Abolins ${ }^{88}$, O.S. AbouZeid ${ }^{158}$, H. Abramowicz ${ }^{153}$, H. Abreu $^{136}$, E. Acerbi ${ }^{89 a, 89 b}$, B.S. Acharya ${ }^{164 a, 164 b}$, L. Adamczyk ${ }^{37}$, D.L. Adams ${ }^{24}$, T.N. Addy ${ }^{56}$, J. Adelman ${ }^{176}$, S. Adomeit ${ }^{98}$, P. Adragna ${ }^{75}$, T. Adye ${ }^{129}$, S. Aefsky ${ }^{22}$, J.A. AguilarSaavedra ${ }^{124 b, a}$, M. Agustoni ${ }^{16}$, M. Aharrouche ${ }^{81}$, S.P. Ahlen ${ }^{21}$, F. Ahles ${ }^{48}$, A. Ahmad ${ }^{148}$, M. $\operatorname{Ahsan}^{40}$, G. Aielli133a,133b, T. Akdogan ${ }^{18 a}$, T.P.A. Åkesson ${ }^{79}$, G. Akimoto ${ }^{155}$, A.V. Akimov ${ }^{94}$, A. Akiyama ${ }^{66}$, M.S. Alam ${ }^{1}$, M.A. Alam ${ }^{76}$, J. Albert ${ }^{169}$, S. Albrand ${ }^{55}$, M. Aleksa ${ }^{29}$, I.N. Aleksandrov ${ }^{64}$, F. Alessandria ${ }^{89 a}$, C. Alexa ${ }^{25 a}$, G. Alexander ${ }^{153}$, G. Alexandre ${ }^{49}$, T. Alexopoulos ${ }^{9}$, M. Alhroob ${ }^{164 a, 164 c}$, M. Aliev ${ }^{15}$, G. Alimonti ${ }^{89 a}$, J. Alison ${ }^{120}$, B.M.M. Allbrooke ${ }^{17}$, P.P. Allport ${ }^{73}$, S.E. Allwood-Spiers ${ }^{53}$, J. Almond ${ }^{82}$, A. Aloisio ${ }^{102 a, 102 b}$, R. Alon ${ }^{172}$, A. Alonso ${ }^{79}$, B. Alvarez Gonzalez ${ }^{88}$, M.G. Alviggi ${ }^{102 a, 102 b}$, K. Amako ${ }^{65}$, C. Amelung 22 , V.V. Ammosov ${ }^{128}$, A. Amorim ${ }^{124 a, b}$, N. Amram ${ }^{153}$, C. Anastopoulos ${ }^{29}$, L.S. Ancu ${ }^{16}$, N. Andari ${ }^{115}$, T. Andeen ${ }^{34}$, C.F. Anders ${ }^{58 b}$, G. Anders ${ }^{58 a}$, K.J. Anderson ${ }^{30}$, A. Andreazza ${ }^{89 a, 89 b}$, V. Andrei ${ }^{58 a}$, X.S. Anduaga ${ }^{70}$, P. Anger ${ }^{43}$, A. Angerami ${ }^{34}$, F. Anghinolfi ${ }^{29}$, A. Anisenkov ${ }^{107}$, N. Anjos ${ }^{124 a}$, A. Annovi ${ }^{47}$, A. Antonaki ${ }^{8}$, M. Antonelli ${ }^{47}$, A. Antonov ${ }^{96}$, J. Antos ${ }^{144 b}$, F. Anulli ${ }^{132 a}$, S. Aoun ${ }^{83}$, L. Aperio Bella ${ }^{4}$, R. Apolle ${ }^{118, c}$, G. Arabidze ${ }^{88}$, I. Aracena ${ }^{143}$, Y. Arai ${ }^{65}$, A.T.H. Arce ${ }^{44}$, S. Arfaoui ${ }^{148}$, J-F. Arguin ${ }^{14}$, E. Arik ${ }^{18 a}$,* M. Arik ${ }^{18 a}$, A.J. Armbruster ${ }^{87}$, O. Arnaez ${ }^{81}$, V. Arnal ${ }^{80}$, C. Arnault ${ }^{115}$, A. Artamonov ${ }^{95}$, G. Artoni ${ }^{132 a, 132 b}$, D. Arutinov ${ }^{20}$, S. Asai ${ }^{155}$,

R. Asfandiyarov ${ }^{173}$, S. Ask ${ }^{27}$, B. Åsman 146a,146b, L. Asquith ${ }^{5}$, K. Assamagan ${ }^{24}$, A. Astbury ${ }^{169}$, B. Aubert ${ }^{4}$, E. Auge ${ }^{115}$, K. Augsten ${ }^{127}$, M. Aurousseau ${ }^{145 a}$, G. Avolio ${ }^{163}$, R. Avramidou ${ }^{9}$, D. Axen ${ }^{168}$, G. Azuelos ${ }^{93, d}$, Y. Azuma ${ }^{155}$, M.A. Baak ${ }^{29}$, G. Baccaglioni89a, C. Bacci ${ }^{134 a, 134 b}$, A.M. Bach ${ }^{14}$, H. Bachacou ${ }^{136}$, K. Bachas ${ }^{29}$, M. Backes ${ }^{49}$, M. Backhaus ${ }^{20}$, E. Badescu ${ }^{25 a}$, P. Bagnaia ${ }^{132 a, 132 b}$, S. Bahinipati ${ }^{2}$, Y. Bai ${ }^{32 a}$, D.C. Bailey ${ }^{158}$, T. Bain ${ }^{158}$, J.T. Baines ${ }^{129}$, O.K. Baker ${ }^{176}$, M.D. Baker ${ }^{24}$, S. Baker ${ }^{77}$, E. Banas ${ }^{38}$, P. Banerjee ${ }^{93}$, Sw. Banerjee ${ }^{173}$, D. Banfi ${ }^{29}$, A. Bangert ${ }^{150}$, V. Bansal ${ }^{169}$, H.S. Bansil ${ }^{17}$, L. Barak ${ }^{172}$, S.P. Baranov ${ }^{94}$, A. Barbaro Galtieri ${ }^{14}$, T. Barber ${ }^{48}$, E.L. Barberio ${ }^{86}$, D. Barberis ${ }^{50 a}, 50 \mathrm{~b}$, M. Barbero ${ }^{20}$, D.Y. Bardin ${ }^{64}$, T. Barillari ${ }^{99}$, M. Barisonzi ${ }^{175}$, T. Barklow ${ }^{143}$, N. Barlow ${ }^{27}$, B.M. Barnett ${ }^{129}$, R.M. Barnett ${ }^{14}$, A. Baroncelli ${ }^{134 a}$, G. Barone ${ }^{49}$, A.J. Barr ${ }^{118}$, F. Barreiro ${ }^{80}$, J. Barreiro Guimarães da $\operatorname{Costa}^{57}$, P. Barrillon ${ }^{115}$, R. Bartoldus ${ }^{143}$, A.E. Barton ${ }^{71}$, V. Bartsch ${ }^{149}$, R.L. Bates ${ }^{53}$, L. Batkova ${ }^{144 a}$, J.R. Batley ${ }^{27}$, A. Battaglia ${ }^{16}$, M. Battistin ${ }^{29}$, F. Bauer ${ }^{136}$, H.S. Bawa ${ }^{143, e}$, S. Beale ${ }^{98}$, T. Beau ${ }^{78}$, P.H. Beauchemin ${ }^{161}$, R. Beccherle ${ }^{50 a}$, P. Bechtle ${ }^{20}$, H.P. Beck ${ }^{16}$, A.K. Becker ${ }^{175}$, S. Becker ${ }^{98}$, M. Beckingham ${ }^{138}$, K.H. Becks ${ }^{175}$, A.J. Beddall ${ }^{18 c}$, A. Beddall ${ }^{18 c}$, S. Bedikian ${ }^{176}$, V.A. Bednyakov ${ }^{64}$, C.P. Bee ${ }^{83}$, M. Begel ${ }^{24}$, S. Behar Harpaz ${ }^{152}$, M. Beimforde ${ }^{99}$, C. Belanger-Champagne ${ }^{85}$, P.J. Bell ${ }^{49}$, W.H. Bell ${ }^{49}$, G. Bella ${ }^{153}$, L. Bellagamba ${ }^{19 a}$, F. Bellina ${ }^{29}$, M. Bellomo ${ }^{29}$, A. Belloni ${ }^{57}$, O. Beloborodova ${ }^{107, f}$, K. Belotskiy ${ }^{96}$, O. Beltramello ${ }^{29}$, O. Benary ${ }^{153}$, D. Benchekroun ${ }^{135 a}$, K. Bendtz ${ }^{146 a, 146 b}$, N. Benekos ${ }^{165}$, Y. Benhammou ${ }^{153}$, E. Benhar Noccioli ${ }^{49}$, J.A. Benitez Garcia ${ }^{159 b}$, D.P. Benjamin ${ }^{44}$, M. Benoit ${ }^{115}$, J.R. Bensinger ${ }^{22}$, K. Benslama ${ }^{130}$, S. Bentvelsen ${ }^{105}$, D. Berge ${ }^{29}$, E. Bergeaas Kuutmann ${ }^{41}$, N. Berger ${ }^{4}$, F. Berghaus ${ }^{169}$, E. Berglund ${ }^{105}$, J. Beringer ${ }^{14}$, P. Bernat ${ }^{77}$, R. Bernhard ${ }^{48}$, C. Bernius ${ }^{24}$, 
T. Berry ${ }^{76}$, C. Bertella ${ }^{83}$, A. Bertin ${ }^{19 a, 19 b}$, F. Bertolucci ${ }^{122 a, 122 b}$, M.I. Besana ${ }^{89 a, 89 b}$, G.J. Besjes ${ }^{104}$, N. Besson ${ }^{136}$, S. Bethke ${ }^{99}$, W. Bhimjii ${ }^{45}$, R.M. Bianchi ${ }^{29}$, M. Bianco ${ }^{72 a, 72 b}$, O. Biebel ${ }^{98}$, S.P. Bieniek ${ }^{77}$, K. Bierwagen ${ }^{54}$, J. Biesiada ${ }^{14}$, M. Biglietti1 ${ }^{134 a}$, H. Bilokon ${ }^{47}$, M. Bindi ${ }^{19 a, 19 b}$, S. Binet ${ }^{115}$, A. Bingul ${ }^{18 c}$, C. Bini ${ }^{132 a, 132 b}$, C. Biscarat ${ }^{178}$, U. Bitenc ${ }^{48}$, K.M. Black ${ }^{21}$, R.E. Blair ${ }^{5}$, J.-B. Blanchard ${ }^{136}$, G. Blanchot ${ }^{29}$, T. Blazek 144a, C. Blocker ${ }^{22}$, J. Blocki ${ }^{38}$, A. Blondel ${ }^{49}$, W. Blum ${ }^{81}$, U. Blumenschein ${ }^{54}$, G.J. Bobbink ${ }^{105}$, V.B. Bobrovnikov ${ }^{107}$, S.S. Bocchetta ${ }^{79}$, A. Bocci ${ }^{44}$, C.R. Boddy ${ }^{118}$, M. Boehler ${ }^{41}$, J. Boek ${ }^{175}$, N. Boelaert ${ }^{35}$, J.A. Bogaerts ${ }^{29}$, A. Bogdanchikov ${ }^{107}$, A. Bogouch ${ }^{90, *}$, C. Bohm ${ }^{146 a}$, J. Bohm ${ }^{125}$, V. Boisvert ${ }^{76}$, T. Bold ${ }^{37}$, V. Boldea ${ }^{25 a}$, N.M. Bolnet ${ }^{136}$, M. Bomben ${ }^{78}$, M. Bona ${ }^{75}$, M. Bondioli ${ }^{163}$, M. Boonekamp ${ }^{136}$, C.N. Booth ${ }^{139}$, S. Bordoni ${ }^{78}$, C. Borer ${ }^{16}$, A. Borisov ${ }^{128}$, G. Borissov ${ }^{71}$, I. Borjanovic ${ }^{12 a}$, M. Borri ${ }^{82}$, S. Borroni ${ }^{87}$, V. Bortolotto ${ }^{134 a, 134 b}$, K. Bos ${ }^{105}$, D. Boscherini ${ }^{19 a}$, M. Bosman ${ }^{11}$, H. Boterenbrood ${ }^{105}$, D. Botterill ${ }^{129}$, J. Bouchami ${ }^{93}$, J. Boudreau ${ }^{123}$, E.V. Bouhova-Thacker ${ }^{71}$, D. Boumediene ${ }^{33}$, C. Bourdarios ${ }^{115}$, N. Bousson ${ }^{83}$, A. Boveia ${ }^{30}$, J. Boyd ${ }^{29}$, I.R. Boyko ${ }^{64}$, I. BozovicJelisavcic $^{12 b}$, J. Bracinik ${ }^{17}$, P. Branchini ${ }^{134 a}$, A. Brandt ${ }^{7}$, G. Brandt ${ }^{118}$, O. Brandt ${ }^{54}$, U. Bratzler ${ }^{156}$, B. Brau ${ }^{84}$, J.E. Brau ${ }^{114}$, H.M. Braun ${ }^{175}$, S.F. Brazzale ${ }^{164 a, 164 c}$, B. Brelier ${ }^{158}$, J. Bremer ${ }^{29}$, K. Brendlinger ${ }^{120}$, R. Brenner ${ }^{166}$, S. Bressler ${ }^{172}$, D. Britton ${ }^{53}$, F.M. Brochu ${ }^{27}$, I. Brock $^{20}$, R. Brock ${ }^{88}$, E. Brodet ${ }^{153}$, F. Broggi ${ }^{89 a}$, C. Bromberg 88 , J. Bronner ${ }^{99}$, G. Brooijmans ${ }^{34}$, T. Brooks ${ }^{76}$, W.K. Brooks ${ }^{31 b}$, G. Brown ${ }^{82}$, H. Brown ${ }^{7}$, P.A. Bruckman de Renstrom ${ }^{38}$, D. Bruncko ${ }^{144 b}$, R. Bruneliere ${ }^{48}$, S. Brunet ${ }^{60}$, A. Bruni $^{19 a}$, G. Bruni ${ }^{19 a}$, M. Bruschi ${ }^{19 a}$, T. Buanes ${ }^{13}$, Q. Buat ${ }^{55}$, F. Bucci ${ }^{49}$, J. Buchanan ${ }^{118}$, P. Buchholz ${ }^{141}$, R.M. Buckingham ${ }^{118}$, A.G. Buckley ${ }^{45}$, S.I. Buda ${ }^{25 a}$, I.A. Budagov ${ }^{64}$, B. Budick ${ }^{108}$, V. Büscher ${ }^{81}$, L. Bugge ${ }^{117}$, O. Bulekov ${ }^{96}$, A.C. Bundock ${ }^{73}$, M. Bunse ${ }^{42}$, T. Buran ${ }^{117}$, H. Burckhart ${ }^{29}$, S. Burdin ${ }^{73}$, T. Burgess ${ }^{13}$, S. Burke ${ }^{129}$, E. Busato ${ }^{33}$, P. Bussey ${ }^{53}$, C.P. Buszello ${ }^{166}$, B. Butler ${ }^{143}$, J.M. Butler ${ }^{21}$, C.M. Buttar ${ }^{53}$, J.M. Butterworth ${ }^{77}$, W. Buttinger ${ }^{27}$, S. Cabrera Urbán ${ }^{167}$, D. Caforio ${ }^{19 a, 19 b}$, O. Cakir ${ }^{3 a}$, P. Calafiura ${ }^{14}$, G. Calderini ${ }^{78}$, P. Calfayan ${ }^{98}$, R. Calkins ${ }^{106}$, L.P. Caloba ${ }^{23 a}$, R. Caloi ${ }^{132 a, 132 b}$, D. Calvet $^{33}$, S. Calvet ${ }^{33}$, R. Camacho Toro ${ }^{33}$, P. Camarri ${ }^{133 a, 133 b}$, D. Cameron ${ }^{117}$, L.M. Caminada ${ }^{14}$, S. Campana ${ }^{29}$, M. Campanelli ${ }^{77}$, V. Canale102a,102b, F. Canelli ${ }^{30, g}$, A. Canepa ${ }^{159 a}$, J. Cantero ${ }^{80}$, R. Cantrill ${ }^{76}$, L. Capasso ${ }^{102 a, 102 b}$, M.D.M. Capeans Garrido ${ }^{29}$, I. Caprini25a, M. Caprini25a, D. Capriotti ${ }^{99}$, M. Capua ${ }^{36 a, 36 b}$,

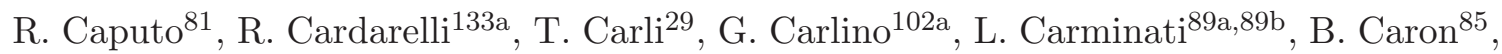
S. Caron ${ }^{104}$, E. Carquin ${ }^{31 b}$, G.D. Carrillo Montoya ${ }^{173}$, A.A. Carter ${ }^{75}$, J.R. Carter ${ }^{27}$, J. Carvalho ${ }^{124 a, h}$, D. Casadei ${ }^{108}$, M.P. Casado ${ }^{11}$, M. Cascella ${ }^{122 a, 122 b}$, C. Caso ${ }^{50 a, 50 b, *}$, A.M. Castaneda Hernandez ${ }^{173, i}$, E. Castaneda-Miranda ${ }^{173}$, V. Castillo Gimenez ${ }^{167}$, N.F. Castro ${ }^{124 a}$, G. Cataldi $^{72 a}$, P. Catastini ${ }^{57}$, A. Catinaccio ${ }^{29}$, J.R. Catmore ${ }^{29}$, A. Cattai $^{29}$, G. Cattani ${ }^{133 a, 133 b}$, S. Caughron ${ }^{88}$, P. Cavalleri ${ }^{78}$, D. Cavalli ${ }^{89 a}$, M. Cavalli-Sforza ${ }^{11}$, V. Cavasinni ${ }^{122 a, 122 b}$, F. Ceradini ${ }^{134 a, 134 b}$, A.S. Cerqueira ${ }^{23 b}$, A. Cerri $^{29}$, L. Cerrito ${ }^{75}$, F. Cerutti ${ }^{47}$, S.A. Cetin ${ }^{18 b}$, A. Chafaq ${ }^{135 a}$, D. Chakraborty ${ }^{106}$, I. Chalupkova ${ }^{126}$, K. Chan $^{2}$, B. Chapleau ${ }^{85}$, J.D. Chapman ${ }^{27}$, J.W. Chapman ${ }^{87}$, E. Chareyre ${ }^{78}$, D.G. Charlton ${ }^{17}$, V. Chavda ${ }^{82}$, C.A. Chavez Barajas ${ }^{29}$, S. Cheatham ${ }^{85}$, S. Chekanov $^{5}$, S.V. Chekulaev ${ }^{159 a}$, G.A. Chelkov ${ }^{64}$, M.A. Chelstowska ${ }^{104}$, C. Chen ${ }^{63}$, H. Chen ${ }^{24}$, S. Chen ${ }^{32 c}$, X. Chen ${ }^{173}$, A. Cheplakov ${ }^{64}$, R. Cherkaoui El Moursli ${ }^{135 e}$, 
V. Chernyatin ${ }^{24}$, E. Cheu $^{6}$, S.L. Cheung ${ }^{158}$, L. Chevalier ${ }^{136}$, G. Chiefari ${ }^{102 a, 102 b}$, L. Chikovani ${ }^{51 a}$, J.T. Childers ${ }^{29}$, A. Chilingarov ${ }^{71}$, G. Chiodini ${ }^{72 a}$, A.S. Chisholm ${ }^{17}$, R.T. Chislett ${ }^{77}$, M.V. Chizhov $^{64}$, G. Choudalakis $^{30}$, S. Chouridou ${ }^{137}$, I.A. Christidi ${ }^{77}$, A. Christov ${ }^{48}$, D. Chromek-Burckhart ${ }^{29}$, M.L. Chu ${ }^{151}$, J. Chudoba ${ }^{125}$, G. Ciapetti ${ }^{132 a, 132 b}$,

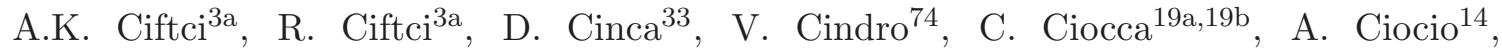
M. Cirilli ${ }^{87}$, P. Cirkovic ${ }^{12 b}$, M. Citterio ${ }^{89 a}$, M. Ciubancan ${ }^{25 a}$, A. Clark ${ }^{49}$, P.J. Clark ${ }^{45}$, W. Cleland ${ }^{123}$, J.C. Clemens ${ }^{83}$, B. Clement ${ }^{55}$, C. Clement ${ }^{146 a, 146 b}$, Y. Coadou ${ }^{83}$, M. Cobal ${ }^{164 a, 164 c}$, A. Coccaro ${ }^{138}$, J. $\operatorname{Cochran}^{63}$, J.G. Cogan ${ }^{143}$, J. Coggeshall ${ }^{165}$, E. Cogneras ${ }^{178}$, J. Colas ${ }^{4}$, A.P. Colijn ${ }^{105}$, N.J. Collins ${ }^{17}$, C. Collins-Tooth ${ }^{53}$, J. Collot ${ }^{55}$, T. Colombo ${ }^{119 a, 119 b}$, G. Colon ${ }^{84}$, P. Conde Muiño ${ }^{124 a}$, E. Coniavitis ${ }^{118}$, M.C. Conidi ${ }^{11}$, S.M. Consonni ${ }^{89 a, 89 b}$, V. Consorti ${ }^{48}$, S. Constantinescu ${ }^{25 a}$, C. Conta ${ }^{119 a, 119 b}$, G. Conti ${ }^{57}$, F. Conventi ${ }^{102 a, j}$, M. Cooke ${ }^{14}$, B.D. Cooper ${ }^{77}$, A.M. Cooper-Sarkar ${ }^{118}$, K. Copic ${ }^{14}$, T. Cornelissen ${ }^{175}$, M. Corradi ${ }^{19 a}$, F. Corriveau ${ }^{85, k}$, A. Cortes-Gonzalez ${ }^{165}$, G. Cortiana ${ }^{99}$, G. Costa ${ }^{89 a}$, M.J. Costa ${ }^{167}$, D. Costanzo ${ }^{139}$, T. Costin $^{30}$, D. Côtéé ${ }^{29}$, L. Courneyea ${ }^{169}$, G. Cowan $^{76}$, C. Cowden ${ }^{27}$, B.E. Cox ${ }^{82}$, K. Cranmer ${ }^{108}, \quad$ F. Crescioli ${ }^{122 a, 122 b}$, M. Cristinziani ${ }^{20}$, G. Crosetti $^{36 a, 36 b}$, R. Crupi ${ }^{72 a, 72 b}$, S. Crépé-Renaudin ${ }^{55}$, C.M. Cuciuc ${ }^{25 a}$, C. Cuenca Almenar ${ }^{176}$, T. Cuhadar Donszelmann ${ }^{139}$, M. Curatolo ${ }^{47}$, C.J. Curtis ${ }^{17}$, C. Cuthbert ${ }^{150}$, P. Cwetanski ${ }^{60}$, H. Czirr ${ }^{141}$, P. Czodrowski ${ }^{43}$, Z. Czyczula ${ }^{176}$, S. D'Auria ${ }^{53}$, M. D'Onofrio ${ }^{73}$, A. D'Orazio ${ }^{132 a, 132 b}$, M.J. Da Cunha Sargedas De Sousa ${ }^{124 a}$, C. Da Via ${ }^{82}$, W. Dabrowski ${ }^{37}$, A. Dafinca ${ }^{118}$, T. Dai ${ }^{87}$, C. Dallapiccola ${ }^{84}, \mathrm{M} \mathrm{Dam}^{35}$, M. Dameri ${ }^{50 a, 50 b}$, D.S. Damiani ${ }^{137}$, H.O. Danielsson ${ }^{29}$, V. Dao ${ }^{49}$, G. Darbo ${ }^{50 a}$, G.L. Darlea ${ }^{25 b}$, W. Davey ${ }^{20}$, T. Davidek ${ }^{126}$, N. Davidson ${ }^{86}$, R. Davidson ${ }^{71}$, E. Davies ${ }^{118, c}$, M. Davies ${ }^{93}$, A.R. Davison ${ }^{77}$, Y. Davygora ${ }^{58 a}$, E. Dawe ${ }^{142}$, I. Dawson ${ }^{139}$, R.K. DayaIshmukhametova $^{22}$, K. De ${ }^{7}$, R. de Asmundis ${ }^{102 a}$, S. De Castro ${ }^{19 a}, 19 b$, S. De Cecco ${ }^{78}$, J. de Graat ${ }^{98}$, N. De Groot ${ }^{104}$, P. de Jong ${ }^{105}$, C. De La Taille ${ }^{115}$, H. De la Torre ${ }^{80}$, F. De Lorenzi ${ }^{63}$, L. de Mora ${ }^{71}$, L. De Nooij ${ }^{105}$, D. De Pedis ${ }^{132 a}$, A. De Salvo ${ }^{132 a}$, U. De Sanctis ${ }^{164 a, 164 c}$, A. De Santo ${ }^{149}$, J.B. De Vivie De Regie ${ }^{115}$, G. De Zorzi ${ }^{132 a, 132 b}$, W.J. Dearnaley ${ }^{71}$, R. Debbe ${ }^{24}$, C. Debenedetti ${ }^{45}$, B. Dechenaux ${ }^{55}$, D.V. Dedovich ${ }^{64}$, J. Degenhardt ${ }^{120}$, C. Del Papa ${ }^{164 a, 164 c}$, J. Del Peso ${ }^{80}$, T. Del Prete ${ }^{122 a, 122 b}$, T. Delemontex ${ }^{55}$, M. Deliyergiyev ${ }^{74}$, A. Dell'Acqua ${ }^{29}$, L. Dell'Asta ${ }^{21}$, M. Della Pietra ${ }^{102 a, j}$, D. della Volpe ${ }^{102 a, 102 b}$, M. Delmastro ${ }^{4}$, P.A. Delsart ${ }^{55}$, C. Deluca ${ }^{105}$, S. Demers ${ }^{176}$, M. Demichev ${ }^{64}$, B. Demirkoz ${ }^{11, l}$, J. Deng ${ }^{163}$, S.P. Denisov ${ }^{128}$, D. Derendarz ${ }^{38}$,

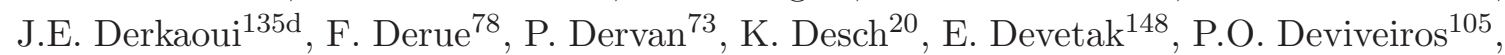
A. Dewhurst ${ }^{129}$, B. DeWilde ${ }^{148}$, S. Dhaliwal ${ }^{158}$, R. Dhullipudi $^{24, m}$, A. Di Ciaccio ${ }^{133 a, 133 b}$, L. Di Ciaccio ${ }^{4}$, A. Di Girolamo ${ }^{29}$, B. Di Girolamo ${ }^{29}$, S. Di Luise ${ }^{134 a, 134 b}$, A. Di Mattia ${ }^{173}$, B. Di Micco ${ }^{29}$, R. Di Nardo ${ }^{47}$, A. Di Simone ${ }^{133 a, 133 b}$, R. Di Sipion ${ }^{19 a, 19 b}$, M.A. Diaz ${ }^{31 a}$, E.B. Diehl ${ }^{87}$, J. Dietrich ${ }^{41}$, T.A. Dietzsch ${ }^{58 a}$, S. Diglio ${ }^{86}$, K. Dindar Yagci ${ }^{39}$, J. Dingfelder ${ }^{20}$,

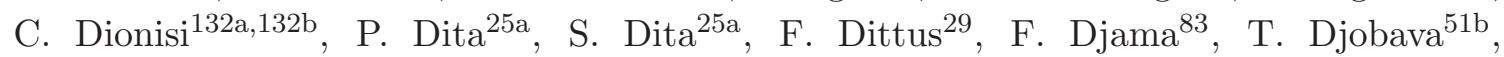
M.A.B. do Vale ${ }^{23 c}$, A. Do Valle Wemans ${ }^{124 a, n}$, T.K.O. Doan ${ }^{4}$, M. Dobbs ${ }^{85}$, R. Dobinson 29,*, D. $\operatorname{Dobos}^{29}$, E. Dobson ${ }^{29, o}$, J. Dodd ${ }^{34}$, C. Doglioni ${ }^{49}$, T. Doherty ${ }^{53}$, Y. Doi ${ }^{65, *}$, J. Dolejsi ${ }^{126}$, I. Dolenc ${ }^{74}$, Z. Dolezal ${ }^{126}$, B.A. Dolgoshein ${ }^{96, *}$, T. Dohmae $^{155}$, M. Donadelli ${ }^{23 d}$, M. Donega ${ }^{120}$, J. Donini ${ }^{33}$, J. Dopke ${ }^{29}$, A. Doria ${ }^{102 a}$, A. Dos Anjos ${ }^{173}$, A. Dotti ${ }^{122 a, 122 b}$, M.T. Dova ${ }^{70}$, A.D. Doxiadis ${ }^{105}$, A.T. Doyle ${ }^{53}$, M. Dris ${ }^{9}$, J. Dubbert ${ }^{99}$, 
S. Dube ${ }^{14}$, E. Duchovni ${ }^{172}$, G. Duckeck ${ }^{98}$, A. Dudarev ${ }^{29}$, F. Dudziak ${ }^{63}$, M. Dührssen ${ }^{29}$, I.P. Duerdoth ${ }^{82}$, L. Duflot ${ }^{115}$, M-A. Dufour ${ }^{85}$, M. Dunford ${ }^{29}$, H. Duran Yildiz ${ }^{3 a}$, R. Duxfield ${ }^{139}$, M. Dwuznik ${ }^{37}$, F. Dydak ${ }^{29}$, M. Düren ${ }^{52}$, J. Ebke ${ }^{98}$, S. Eckweiler ${ }^{81}$, K. Edmonds ${ }^{81}$, C.A. Edwards ${ }^{76}$, N.C. Edwards ${ }^{53}$, W. Ehrenfeld ${ }^{41}$, T. Eifert ${ }^{143}$, G. Eigen ${ }^{13}$, K. Einsweiler ${ }^{14}$, E. Eisenhandler ${ }^{75}$, T. Ekelof ${ }^{166}$, M. El Kacimi ${ }^{135 c}$, M. Ellert ${ }^{166}$, S. Elles ${ }^{4}$, F. Ellinghaus ${ }^{81}$, K. Ellis ${ }^{75}$, N. Ellis ${ }^{29}$, J. Elmsheuser ${ }^{98}$, M. Elsing ${ }^{29}$, D. Emeliyanov ${ }^{129}$, R. Engelmann ${ }^{148}$, A. $\operatorname{Engl}^{98}$, B. Epp ${ }^{61}$, A. Eppig ${ }^{87}$, J. Erdmann ${ }^{54}$, A. Ereditato $^{16}$, D. Eriksson ${ }^{146 a}$, J. Ernst ${ }^{1}$, M. Ernst ${ }^{24}$, J. Ernwein ${ }^{136}$, D. Errede ${ }^{165}$, S. Errede ${ }^{165}$, E. Ertel ${ }^{81}$, M. Escalier ${ }^{115}$, C. Escobar ${ }^{123}$, X. Espinal Curull ${ }^{11}$, B. Esposito ${ }^{47}$, F. Etienne ${ }^{83}$, A.I. Etienvre ${ }^{136}$, E. Etzion ${ }^{153}$, D. Evangelakou ${ }^{54}$, H. Evans ${ }^{60}$, L. Fabbri ${ }^{19 a, 19 b}$, C. Fabre $^{29}$, R.M. Fakhrutdinov ${ }^{128}$, S. Falciano ${ }^{132 a}$, Y. Fang ${ }^{173}$, M. Fanti ${ }^{89 a, 89 b}$, A. Farbin ${ }^{7}$, A. Farilla ${ }^{134 a}$, J. Farley ${ }^{148}$, T. Farooque ${ }^{158}$, S. Farrell ${ }^{163}$, S.M. Farrington ${ }^{118}$, P. Farthouat ${ }^{29}$, P. Fassnacht ${ }^{29}$, D. Fassouliotis ${ }^{8}$, B. Fatholahzadeh ${ }^{158}$, A. Favareto ${ }^{89 a, 89 b}$, L. Fayard ${ }^{115}$, S. Fazio ${ }^{36 a}, 36 \mathrm{~b}$, R. Febbraro ${ }^{33}$, P. Federic ${ }^{144 a}$, O.L. Fedin ${ }^{121}$, W. Fedorko ${ }^{88}$, M. Fehling-Kaschek ${ }^{48}$, L. Feligioni ${ }^{83}$, D. Fellmann ${ }^{5}$, C. Feng ${ }^{32 d}$, E.J. Feng ${ }^{5}$, A.B. Fenyuk ${ }^{128}$, J. Ferencei ${ }^{144 \mathrm{~b}}$, W. Fernando ${ }^{5}$, S. Ferrag ${ }^{53}$, J. Ferrando ${ }^{53}$, V. Ferrara ${ }^{41}$, A. Ferrari ${ }^{166}$, P. Ferrari ${ }^{105}$, R. Ferrari ${ }^{119 a}$, D.E. Ferreira de Lima $^{53}$, A. Ferrer ${ }^{167}$, D. Ferrere ${ }^{49}$, C. Ferretti ${ }^{87}$, A. Ferretto Parodi ${ }^{50 a, 50 b}$, M. Fiascaris ${ }^{30}$, F. Fiedler ${ }^{81}$, A. Filipčič ${ }^{74}$, F. Filthaut ${ }^{104}$, M. Fincke-Keeler ${ }^{169}$, M.C.N. Fiolhais ${ }^{124 a, h}$, L. Fiorini ${ }^{167}$, A. Firan ${ }^{39}$, G. Fischer ${ }^{41}$, M.J. Fisher ${ }^{109}$, M. Flechl ${ }^{48}$, I. Fleck ${ }^{141}$, J. Fleckner ${ }^{81}$, P. Fleischmann ${ }^{174}$, S. Fleischmann ${ }^{175}$, T. Flick ${ }^{175}$, A. Floderus ${ }^{79}$, L.R. Flores Castillo ${ }^{173}$, M.J. Flowerdew ${ }^{99}$, T. Fonseca Martin ${ }^{16}$, A. Formica ${ }^{136}$, A. Forti ${ }^{82}$, D. Fortin ${ }^{159 a}$, D. Fournier ${ }^{115}$, H. Fox ${ }^{71}$, P. Francavilla ${ }^{11}$, S. Franchino ${ }^{119 a, 119 b}$, D. Francis ${ }^{29}$, T. Frank ${ }^{172}$, S. Franz ${ }^{29}$, M. Fraternali ${ }^{119 a, 119 b}$, S. Fratina ${ }^{120}$, S.T. French ${ }^{27}$, C. Friedrich ${ }^{41}$, F. Friedrich ${ }^{43}$, R. Froeschl ${ }^{29}$, D. Froidevaux ${ }^{29}$, J.A. Frost ${ }^{27}$, C. Fukunaga ${ }^{156}$, E. Fullana Torregrosa ${ }^{29}$, B.G. Fulsom ${ }^{143}$, J. Fuster ${ }^{167}$, C. Gabaldon ${ }^{29}$, O. Gabizon ${ }^{172}$, T. Gadfort ${ }^{24}$, S. Gadomski ${ }^{49}$, G. Gagliardi ${ }^{50 a, 50 b}$, P. Gagnon ${ }^{60}$, C. Galea ${ }^{98}$, E.J. Gallas ${ }^{118}$, V. Gallo ${ }^{16}$, B.J. Gallop ${ }^{129}$, P. Gallus ${ }^{125}$, K.K. Gan ${ }^{109}$, Y.S. Gao ${ }^{143, e}$, A. Gaponenko ${ }^{14}$, F. Garberson ${ }^{176}$, M. GarciaSciveres $^{14}$, C. García ${ }^{167}$, J.E. García Navarro ${ }^{167}$, R.W. Gardner ${ }^{30}$, N. Garelli29, H. Garitaonandia ${ }^{105}$, V. Garonne ${ }^{29}$, J. Garvey ${ }^{17}$, C. Gatti ${ }^{47}$, G. Gaudio ${ }^{119 a}$, B. Gaur ${ }^{141}$, L. Gauthier ${ }^{136}$, P. Gauzzi ${ }^{132 a, 132 b}$, I.L. Gavrilenko ${ }^{94}$, C. Gay ${ }^{168}$, G. Gaycken ${ }^{20}$, E.N. Gazis ${ }^{9}$, P. Ge ${ }^{32 d}$, Z. Gecse ${ }^{168}$, C.N.P. Gee ${ }^{129}$, D.A.A. Geerts ${ }^{105}$, Ch. GeichGimbel $^{20}$, K. Gellerstedt ${ }^{146 a, 146 b}$, C. Gemme ${ }^{50 a}$, A. Gemmell ${ }^{53}$, M.H. Genest ${ }^{55}$, S. Gentile $\mathrm{e}^{132 \mathrm{a}, 132 \mathrm{~b}}$, M. George $\mathrm{F}^{54}$, S. George ${ }^{76}$, P. Gerlach ${ }^{175}$, A. Gershon ${ }^{153}$, C. Geweniger ${ }^{58 a}$, H. Ghazlane ${ }^{135 b}$, N. Ghodbane ${ }^{33}$, B. Giacobbe ${ }^{19 a}$, S. Giagu ${ }^{132 a, 132 b}$, V. Giakoumopoulou ${ }^{8}$, V. Giangiobbe ${ }^{11}$, F. Gianotti ${ }^{29}$, B. Gibbard ${ }^{24}$, A. Gibson ${ }^{158}$, S.M. Gibson ${ }^{29}$, D. Gillberg ${ }^{28}$, A.R. Gillman ${ }^{129}$, D.M. Gingrich ${ }^{2, d}$, J. Ginzburg ${ }^{153}$, N. Giokaris ${ }^{8}$, M.P. Giordani ${ }^{164 c}$, R. Giordano ${ }^{102 a, 102 b}$, F.M. Giorgi ${ }^{15}$, P. Giovannini ${ }^{99}$, P.F. Giraud ${ }^{136}$, D. Giugni ${ }^{89 a}$, M. Giunta ${ }^{93}$, P. Giusti ${ }^{19 a}$, B.K. Gjelsten ${ }^{117}$, L.K. Gladilin ${ }^{97}$, C. Glasman ${ }^{80}$, J. Glatzer ${ }^{48}$, A. Glazov ${ }^{41}$, K.W. Glitza ${ }^{175}$, G.L. Glonti ${ }^{64}$, J.R. Goddard ${ }^{75}$, J. Godfrey ${ }^{142}$, J. Godlewski ${ }^{29}$, M. Goebel ${ }^{41}$, T. Göpfert ${ }^{43}$, C. Goeringer ${ }^{81}$, C. Gössling ${ }^{42}$, S. Goldfarb ${ }^{87}$, T. Golling ${ }^{176}$, A. Gomes ${ }^{124 a, b}$, L.S. Gomez Fajardo ${ }^{41}$, R. Gonçalo ${ }^{76}$, J. Goncalves Pinto Firmino Da Costa $^{41}$, L. Gonella ${ }^{20}$, S. Gonzalez ${ }^{173}$, S. González 
de la Hoz ${ }^{167}$, G. Gonzalez Parra ${ }^{11}$, M.L. Gonzalez Silva ${ }^{26}$, S. Gonzalez-Sevilla ${ }^{49}$, J.J. Goodson ${ }^{148}$, L. Goossens ${ }^{29}$, P.A. Gorbounov ${ }^{95}$, H.A. Gordon ${ }^{24}$, I. Gorelov ${ }^{103}$, G. Gorfine ${ }^{175}$, B. Gorini ${ }^{29}$, E. Gorini ${ }^{72 a, 72 b}$, A. Gorišek ${ }^{74}$, E. Gornicki ${ }^{38}$, B. Gosdzik ${ }^{41}$, A.T. Goshaw ${ }^{5}$, M. Gosselink ${ }^{105}$, M.I. Gostkin ${ }^{64}$, I. Gough Eschrich ${ }^{163}$, M. Gouighri ${ }^{135 a}$, D. Goujdami ${ }^{135 c}$, M.P. Goulette ${ }^{49}$, A.G. Goussiou ${ }^{138}$, C. Goy ${ }^{4}$, S. Gozpinar ${ }^{22}$, I. Grabowska-Bold ${ }^{37}$, P. Grafström ${ }^{29}$, K-J. Grahn ${ }^{41}$, F. Grancagnolo ${ }^{72 a}$, S. Grancagnolo ${ }^{15}$, V. Grassi ${ }^{148}$, V. Gratchev ${ }^{121}$, N. Grau ${ }^{34}$, H.M. Gray ${ }^{29}$, J.A. Gray ${ }^{148}$, E. Graziani ${ }^{134 a}$, O.G. Grebenyuk ${ }^{121}$, T. Greenshaw ${ }^{73}$, Z.D. Greenwood ${ }^{24, m}$, K. Gregersen ${ }^{35}$, I.M. Gregor ${ }^{41}$, P. Grenier ${ }^{143}$, J. Griffiths ${ }^{138}$, N. Grigalashvili ${ }^{64}$, A.A. Grillo ${ }^{137}$, S. Grinstein ${ }^{11}$, Y.V. Grishkevich ${ }^{97}$, J.-F. Grivaz ${ }^{115}$, E. Gross ${ }^{172}$, J. Grosse-Knetter ${ }^{54}$, J. GrothJensen $^{172}$, K. Grybel ${ }^{141}$, D. Guest ${ }^{176}$, C. Guicheney ${ }^{33}$, A. Guida ${ }^{72 a, 72 b}$, S. Guindon ${ }^{54}$, U. Gul ${ }^{53}$, H. Guler ${ }^{85, p}$, J. Gunther ${ }^{125}$, B. Guo ${ }^{158}$, J. Guo ${ }^{34}$, P. Gutierrez ${ }^{111}$, N. Guttman ${ }^{153}$, O. Gutzwiller ${ }^{173}$, C. Guyot ${ }^{136}$, C. Gwenlan ${ }^{118}$, C.B. Gwilliam ${ }^{73}$, A. $\operatorname{Haas}^{143}$, S. $\operatorname{Haas}^{29}$, C. Haber ${ }^{14}$, H.K. Hadavand ${ }^{39}$, D.R. Hadley ${ }^{17}$, P. Haefner ${ }^{20}$, F. $\operatorname{Hahn}^{29}$, S. Haider ${ }^{29}$, Z. Hajduk ${ }^{38}$, H. Hakobyan ${ }^{177}$, D. Hall ${ }^{118}$, J. Haller ${ }^{54}$, K. Hamacher ${ }^{175}$, P. Hamal ${ }^{113}$, M. Hamer ${ }^{54}$, A. Hamilton ${ }^{145 b, q}$, S. Hamilton ${ }^{161}$, L. Han ${ }^{32 b}$, K. Hanagaki ${ }^{116}$, K. Hanawa ${ }^{160}$, M. Hance ${ }^{14}$, C. Handel ${ }^{81}$, P. Hanke ${ }^{58 a}$, J.R. Hansen ${ }^{35}$,

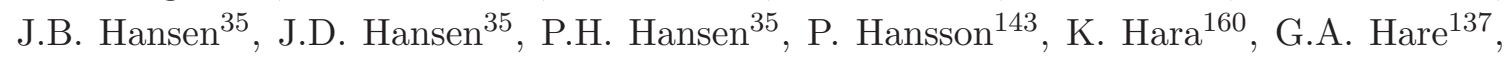
T. Harenberg ${ }^{175}$, S. Harkusha ${ }^{90}$, D. Harper ${ }^{87}$, R.D. Harrington ${ }^{45}$, O.M. Harris ${ }^{138}$, K. Harrison ${ }^{17}$, J. Hartert ${ }^{48}$, F. Hartjes ${ }^{105}$, T. Haruyama ${ }^{65}$, A. Harvey ${ }^{56}$, S. Hasegawa ${ }^{101}$, Y. Hasegawa ${ }^{140}$, S. Hassani ${ }^{136}$, S. Haug ${ }^{16}$, M. Hauschild ${ }^{29}$, R. Hauser ${ }^{88}$, M. Havranek ${ }^{20}$, C.M. Hawkes ${ }^{17}$, R.J. Hawkings ${ }^{29}$, A.D. Hawkins ${ }^{79}$, D. Hawkins ${ }^{163}$, T. Hayakawa ${ }^{66}$, T. Hayashi ${ }^{160}$, D. Hayden ${ }^{76}$, C.P. Hays ${ }^{118}$, H.S. Hayward ${ }^{73}$, S.J. Haywood ${ }^{129}$, M. He ${ }^{32 d}$, S.J. Head ${ }^{17}$, V. Hedberg ${ }^{79}$, L. Heelan ${ }^{7}$, S. Heim ${ }^{88}$, B. Heinemann ${ }^{14}$, S. Heisterkamp ${ }^{35}$, L. Helary ${ }^{4}$, C. Heller ${ }^{98}$, M. Heller ${ }^{29}$, S. Hellman ${ }^{146 a, 146 b}$, D. Hellmich ${ }^{20}$, C. Helsens ${ }^{11}$, R.C.W. Henderson ${ }^{71}$, M. Henke ${ }^{58 a}$, A. Henrichs ${ }^{54}$, A.M. Henriques Correia ${ }^{29}$, S. HenrotVersille $^{115}$, C. Hensel ${ }^{54}$, T. Henß ${ }^{175}$, C.M. Hernandez ${ }^{7}$, Y. Hernández Jiménez ${ }^{167}$, R. Herrberg ${ }^{15}$, G. Herten ${ }^{48}$, R. Hertenberger ${ }^{98}$, L. Hervas ${ }^{29}$, G.G. Hesketh ${ }^{77}$, N.P. Hessey ${ }^{105}$, E. Higón-Rodriguez ${ }^{167}$, J.C. Hill ${ }^{27}$, K.H. Hiller ${ }^{41}$, S. Hillert ${ }^{20}$, S.J. Hillier ${ }^{17}$, I. Hinchliffe ${ }^{14}$, E. Hines ${ }^{120}$, M. Hirose ${ }^{116}$, F. Hirsch ${ }^{42}$, D. Hirschbuehl ${ }^{175}$, J. Hobbs ${ }^{148}$, N. $\operatorname{Hod}^{153}$, M.C. Hodgkinson ${ }^{139}$, P. Hodgson ${ }^{139}$, A. Hoecker ${ }^{29}$, M.R. Hoeferkamp ${ }^{103}$, J. Hoffman ${ }^{39}$, D. Hoffmann ${ }^{83}$, M. Hohlfeld ${ }^{81}$, M. Holder ${ }^{141}$, S.O. Holmgren ${ }^{146 a}$, T. Holy ${ }^{127}$, J.L. Holzbauer ${ }^{88}$, T.M. Hong ${ }^{120}$, L. Hooft van Huysduynen ${ }^{108}$, C. Horn ${ }^{143}$, S. Horner ${ }^{48}$,

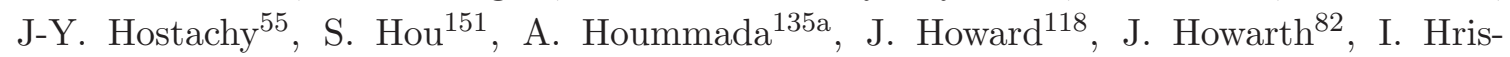
tova ${ }^{15}$, J. Hrivnac ${ }^{115}$, T. Hryn'ova ${ }^{4}$, P.J. Hsu ${ }^{81}$, S.-C. Hsu ${ }^{14}$, Z. Hubacek ${ }^{127}$, F. Hubaut ${ }^{83}$, F. Huegging ${ }^{20}$, A. Huettmann ${ }^{41}$, T.B. Huffman ${ }^{118}$, E.W. Hughes ${ }^{34}$, G. Hughes ${ }^{71}$, M. Huhtinen ${ }^{29}$, M. Hurwitz ${ }^{14}$, U. Husemann ${ }^{41}$, N. Huseynov ${ }^{64, r}$, J. Huston ${ }^{88}$, J. Huth ${ }^{57}$, G. Iacobucci ${ }^{49}$, G. Iakovidis ${ }^{9}$, M. Ibbotson ${ }^{82}$, I. Ibragimov ${ }^{141}$, L. IconomidouFayard $^{115}$, J. Idarraga ${ }^{115}$, P. Iengo ${ }^{102 a}$, O. Igonkina ${ }^{105}$, Y. Ikegami ${ }^{65}$, M. Ikeno ${ }^{65}$, D. Iliadis ${ }^{154}$, N. Ilic ${ }^{158}$, T. Ince ${ }^{20}$, J. Inigo-Golfin ${ }^{29}$, P. Ioannou ${ }^{8}$, M. Iodice ${ }^{134 a}$, K. Iordanidou ${ }^{8}$, V. Ippolito ${ }^{132 a, 132 b}$, A. Irles Quiles ${ }^{167}$, C. Isaksson ${ }^{166}$, A. Ishikawa ${ }^{66}$, M. Ishino ${ }^{67}$, R. Ishmukhametov ${ }^{39}$, C. Issever ${ }^{118}$, S. Istin ${ }^{18 a}$, A.V. Ivashin ${ }^{128}$, W. Iwanski ${ }^{38}$, H. Iwasaki ${ }^{65}$, J.M. $\operatorname{Izen}^{40}$, V. Izzo ${ }^{102 a}$, B. Jackson ${ }^{120}$, J.N. Jackson ${ }^{73}$, P. Jackson ${ }^{143}$, 
M.R. Jaekel ${ }^{29}$, V. Jain ${ }^{60}$, K. Jakobs ${ }^{48}$, S. Jakobsen ${ }^{35}$, T. Jakoubek ${ }^{125}$, J. Jakubek ${ }^{127}$, D.K. Jana ${ }^{111}$, E. Jansen ${ }^{77}$, H. Jansen ${ }^{29}$, A. Jantsch ${ }^{99}$, M. Janus ${ }^{48}$, G. Jarlskog ${ }^{79}$, L. Jeanty ${ }^{57}$, I. Jen-La Plante ${ }^{30}$, P. Jenni ${ }^{29}$, A. Jeremie ${ }^{4}$, P. Jež ${ }^{35}$, S. Jézéquel ${ }^{4}$, M.K. Jha ${ }^{19 a}$, H. Ji ${ }^{173}$, W. Ji ${ }^{81}$, J. Jia ${ }^{148}$, Y. Jiang ${ }^{32 b}$, M. Jimenez Belenguer ${ }^{41}$, S. Jin ${ }^{32 a}$, O. Jinnouchi ${ }^{157}$, M.D. Joergensen ${ }^{35}$, D. Joffe ${ }^{39}$, M. Johansen ${ }^{146 a, 146 b}$, K.E. Johansson ${ }^{146 a}$, P. Johansson ${ }^{139}$, S. Johnert ${ }^{41}$, K.A. Johns ${ }^{6}$, K. Jon-And ${ }^{146 a, 146 b}$, G. Jones ${ }^{170}$, R.W.L. Jones ${ }^{71}$, T.J. Jones ${ }^{73}$, C. Joram ${ }^{29}$, P.M. Jorge ${ }^{124 a}$, K.D. Joshi ${ }^{82}$, J. Jovicevic ${ }^{147}$, T. Jovin ${ }^{12 b}$, X. Ju ${ }^{173}$, C.A. Jung ${ }^{42}$, R.M. Jungst ${ }^{29}$, V. Juranek ${ }^{125}$, P. Jussel ${ }^{61}$, A. Juste Rozas ${ }^{11}$, S. Kabana ${ }^{16}$, M. Kaci ${ }^{167}$, A. Kaczmarska ${ }^{38}$, P. Kadlecik ${ }^{35}$, M. Kado ${ }^{115}$, H. Kagan ${ }^{109}$, M. $\operatorname{Kagan}^{57}$, E. Kajomovitz ${ }^{152}$, S. Kalinin ${ }^{175}$, L.V. Kalinovskaya ${ }^{64}$, S. Kama ${ }^{39}$, N. Kanaya ${ }^{155}$, M. Kaneda ${ }^{29}$, S. Kaneti ${ }^{27}$, T. Kanno ${ }^{157}$, V.A. Kantserov ${ }^{96}$, J. Kanzaki ${ }^{65}$, B. Kaplan ${ }^{176}$, A. Kapliy ${ }^{30}$, J. Kaplon ${ }^{29}$, D. Kar ${ }^{53}$, M. Karagounis ${ }^{20}$, K. Karakostas ${ }^{9}$, M. Karnevskiy ${ }^{41}$, V. Kartvelishvili ${ }^{71}$, A.N. Karyukhin ${ }^{128}$, L. Kashif ${ }^{173}$, G. Kasieczka ${ }^{58 b}$, R.D. Kass ${ }^{109}$, A. Kastanas ${ }^{13}$, M. Kataoka ${ }^{4}$, Y. Kataoka ${ }^{155}$, E. Katsoufis ${ }^{9}$, J. Katzy ${ }^{41}$, V. Kaushik ${ }^{6}$, K. Kawagoe ${ }^{69}$, T. Kawamoto ${ }^{155}$, G. Kawamura ${ }^{81}$, M.S. Kayl ${ }^{105}$, V.A. Kazanin ${ }^{107}$, M.Y. Kazarinov ${ }^{64}$, R. Keeler ${ }^{169}$, R. Kehoe $^{39}$, M. Keil ${ }^{54}$, G.D. Kekelidze ${ }^{64}$, J.S. Keller ${ }^{138}$, M. Kenyon ${ }^{53}$, O. Kepka ${ }^{125}$, N. Kerschen ${ }^{29}$, B.P. Kerševan ${ }^{74}$, S. Kersten ${ }^{175}$, K. Kessoku ${ }^{155}$, J. Keung ${ }^{158}$, F. Khalil-zada ${ }^{10}$, H. Khandanyan ${ }^{165}$, A. Khanov ${ }^{112}$, D. Kharchenko ${ }^{64}$, A. Khodinov ${ }^{96}$, A. Khomich ${ }^{58 a}$, T.J. Khoo ${ }^{27}$, G. Khoriauli20, A. Khoroshilov ${ }^{175}$, V. Khovanskiy ${ }^{95}$, E. Khramov ${ }^{64}$, J. Khubua ${ }^{51 b}$, H. Kim ${ }^{146 a, 146 b}$, M.S. Kim ${ }^{2}$, S.H. Kim ${ }^{160}$, N. Kimura ${ }^{171}$, O. Kind ${ }^{15}$, B.T. King ${ }^{73}$, M. King ${ }^{66}$, R.S.B. King ${ }^{118}$, J. Kirk ${ }^{129}$, A.E. Kiryunin ${ }^{99}$, T. Kishimoto ${ }^{66}$, D. Kisielewska ${ }^{37}$, T. Kittelmann ${ }^{123}$, E. Kladiva ${ }^{144 \mathrm{~b}}$, M. Klein ${ }^{73}$, U. Klein ${ }^{73}$, K. Kleinknecht ${ }^{81}$, M. Klemetti ${ }^{85}$, A. Klier ${ }^{172}$, P. Klimek ${ }^{146 a, 146 b}$, A. Klimentov ${ }^{24}$, R. Klingenberg ${ }^{42}$, J.A. Klinger ${ }^{82}$, E.B. Klinkby ${ }^{35}$, T. Klioutchnikova ${ }^{29}$, P.F. Klok ${ }^{104}$, S. Klous ${ }^{105}$, E.-E. Kluge ${ }^{58 a}$, T. Kluge ${ }^{73}$, P. Kluit ${ }^{105}$, S. Kluth ${ }^{99}$, N.S. Knecht ${ }^{158}$, E. Kneringer ${ }^{61}$, E.B.F.G. Knoops ${ }^{83}$, A. Knue ${ }^{54}$, B.R. Ko ${ }^{44}$, T. Kobayashi ${ }^{155}$, M. Kobel $^{43}$, M. Kocian ${ }^{143}$, P. Kodys ${ }^{126}$, K. Köneke ${ }^{29}$, A.C. König ${ }^{104}$, S. Koenig ${ }^{81}$, L. Köpke ${ }^{81}$, F. Koetsveld ${ }^{104}$, P. Koevesarki ${ }^{20}$, T. Koffas ${ }^{28}$, E. Koffeman ${ }^{105}$, L.A. Kogan ${ }^{118}$, S. Kohlmann ${ }^{175}$, F. Kohn ${ }^{54}$, Z. Kohout ${ }^{127}$, T. Kohriki ${ }^{65}$, T. Koi ${ }^{143}$, G.M. Kolachev ${ }^{107}$, H. Kolanoski ${ }^{15}$, V. Kolesnikov ${ }^{64}$, I. Koletsou ${ }^{89 a}$, J. Koll ${ }^{88}$, M. Kollefrath ${ }^{48}$, A.A. Komar ${ }^{94}$, Y. Komori ${ }^{155}$, T. Kondo ${ }^{65}$, T. Kono ${ }^{41, s}$, A.I. Kononov ${ }^{48}$, R. Konoplich ${ }^{108, t}$, N. Konstantinidis ${ }^{77}$, S. Koperny ${ }^{37}$, K. Korcy ${ }^{38}$, K. Kordas ${ }^{154}$, A. Korn ${ }^{118}$, A. Korol ${ }^{107}$, I. Korolkov ${ }^{11}$, E.V. Korolkova ${ }^{139}$, V.A. Korotkov ${ }^{128}$, O. Kortner ${ }^{99}$, S. Kortner ${ }^{99}$, V.V. Kostyukhin ${ }^{20}$, S. Kotov ${ }^{99}$, V.M. Kotov ${ }^{64}$, A. Kotwal ${ }^{44}$, C. Kourkoumelis ${ }^{8}$, V. Kouskoura ${ }^{154}$, A. Koutsman 159a, R. Kowalewski ${ }^{169}$, T.Z. Kowalski ${ }^{37}$, W. Kozanecki ${ }^{136}$, A.S. Kozhin ${ }^{128}$, V. Kral ${ }^{127}$, V.A. Kramarenko ${ }^{97}$, G. Kramberger ${ }^{74}$, M.W. Krasny ${ }^{78}$, A. Krasznahorkay ${ }^{108}$, J. Kraus ${ }^{88}$, J.K. Kraus ${ }^{20}$, S. Kreiss ${ }^{108}$, F. Krejci' ${ }^{127}$, J. Kretzschmar ${ }^{73}$, N. Krieger ${ }^{54}$, P. Krieger ${ }^{158}$, K. Kroeninger ${ }^{54}$, H. Kroha ${ }^{99}$, J. Kroll ${ }^{120}$, J. Kroseberg ${ }^{20}$, J. Krstic ${ }^{12 a}$, U. Kruchonak ${ }^{64}$, H. Krüger ${ }^{20}$, T. Kruker ${ }^{16}$, N. Krumnack ${ }^{63}$, Z.V. Krumshteyn ${ }^{64}$, A. Kruth ${ }^{20}$, T. Kubota ${ }^{86}$, S. Kuday ${ }^{3 a}$, S. Kuehn ${ }^{48}$, A. Kugel ${ }^{58 c}$, T. Kuhl ${ }^{41}$, D. Kuhn ${ }^{61}$, V. Kukhtin ${ }^{64}$, Y. Kulchitsky ${ }^{90}$, S. Kuleshov ${ }^{31 b}$, C. Kummer ${ }^{98}$, M. Kuna ${ }^{78}$, J. Kunkle ${ }^{120}$, A. Kupco ${ }^{125}$, H. Kurashige ${ }^{66}$, M. Kurata ${ }^{160}$, Y.A. Kurochkin ${ }^{90}$, V. Kus ${ }^{125}$, E.S. Kuwertz ${ }^{147}$, 
M. Kuze ${ }^{157}$, J. Kvita ${ }^{142}$, R. Kwee ${ }^{15}$, A. La Rosa ${ }^{49}$, L. La Rotonda ${ }^{36 a, 36 b}$, L. Labarga ${ }^{80}$, J. Labbe ${ }^{4}$, S. Lablak ${ }^{135 a}$, C. Lacasta ${ }^{167}$, F. Lacava ${ }^{132 a, 132 b}$, H. Lacker ${ }^{15}$, D. Lacour ${ }^{78}$, V.R. Lacuesta ${ }^{167}$, E. Ladygin ${ }^{64}$, R. Lafaye $^{4}$, B. Laforge ${ }^{78}$, T. Lagouri ${ }^{80}$, S. Lai ${ }^{48}$, E. Laisne ${ }^{55}$, M. Lamanna ${ }^{29}$, L. Lambourne ${ }^{77}$, C.L. Lampen ${ }^{6}$, W. Lampl ${ }^{6}$, E. Lancon ${ }^{136}$, U. Landgraf ${ }^{48}$, M.P.J. Landon ${ }^{75}$, J.L. Lane ${ }^{82}$, C. Lange ${ }^{41}$, A.J. Lankford ${ }^{163}$, F. Lanni ${ }^{24}$,

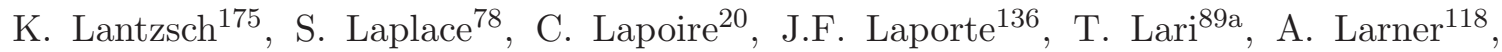
M. Lassnig ${ }^{29}$, P. Laurelli ${ }^{47}$, V. Lavorini ${ }^{36 a, 36 b}$, W. Lavrijsen ${ }^{14}$, P. Laycock ${ }^{73}$, O. Le Dortz ${ }^{78}$, E. Le Guirriec ${ }^{83}$, C. Le Maner ${ }^{158}$, E. Le Menedeu ${ }^{11}$, T. LeCompte ${ }^{5}$, F. Ledroit-Guillon ${ }^{55}$, H. Lee ${ }^{105}$, J.S.H. Lee ${ }^{116}$, S.C. Lee ${ }^{151}$, L. Lee ${ }^{176}$, M. Lefebvre ${ }^{169}$, M. Legendre ${ }^{136}$, B.C. LeGeyt ${ }^{120}$, F. Legger ${ }^{98}$, C. Leggett ${ }^{14}$, M. Lehmacher ${ }^{20}$, G. Lehmann Miotto ${ }^{29}$, X. Lei $^{6}$, M.A.L. Leite ${ }^{23 d}$, R. Leitner ${ }^{126}$, D. Lellouch ${ }^{172}$, B. Lemmer ${ }^{54}$, V. Lendermann ${ }^{58 a}$, K.J.C. Leney ${ }^{145 b}$, T. Lenz ${ }^{105}$, G. Lenzen ${ }^{175}$, B. Lenzi ${ }^{29}$, K. Leonhardt ${ }^{43}$, S. Leontsinis ${ }^{9}$, F. Lepold ${ }^{58 a}$, C. Leroy ${ }^{93}$, J-R. Lessard ${ }^{169}$, C.G. Lester ${ }^{27}$, C.M. Lester ${ }^{120}$, J. Levêque ${ }^{4}$, D. Levin ${ }^{87}$, L.J. Levinson ${ }^{172}$, A. Lewis ${ }^{118}$, G.H. Lewis ${ }^{108}$, A.M. Leyko ${ }^{20}$, M. Leyton ${ }^{15}$, B. $\mathrm{Li}^{83}$, H. $\mathrm{Li}^{173, u}, \mathrm{~S} \cdot \mathrm{Li}^{32 \mathrm{~b}, v}, \mathrm{X} . \mathrm{Li}^{87}$, Z. Liang${ }^{118, w}, \mathrm{H} \cdot \mathrm{LiaO}^{33}$, B. Liberti ${ }^{133 \mathrm{a}}$,

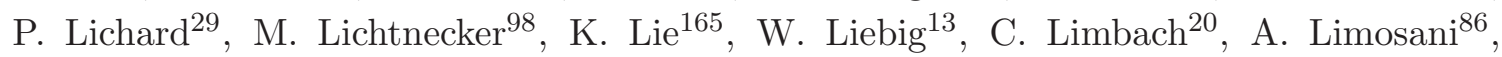
M. Limper ${ }^{62}$, S.C. $\operatorname{Lin}^{151, x}$, F. Linde ${ }^{105}$, J.T. Linnemann ${ }^{88}$, E. Lipeles ${ }^{120}$, A. Lipniacka ${ }^{13}$, T.M. Liss ${ }^{165}$, D. Lissauer ${ }^{24}$, A. Lister ${ }^{49}$, A.M. Litke ${ }^{137}$, C. Liu ${ }^{28}$, D. Liu ${ }^{151}$, H. Liu ${ }^{87}$,

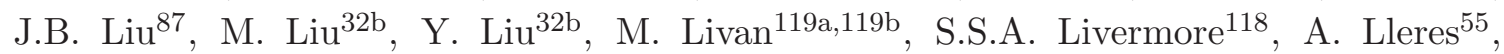
J. Llorente Merino ${ }^{80}$, S.L. Lloyd ${ }^{75}$, E. Lobodzinska ${ }^{41}$, P. Loch $^{6}$, W.S. Lockman ${ }^{137}$, T. Loddenkoetter ${ }^{20}$, F.K. Loebinger ${ }^{82}$, A. Loginov ${ }^{176}$, C.W. Loh $^{168}$, T. Lohse ${ }^{15}$, K. Lohwasser ${ }^{48}$, M. Lokajicek ${ }^{125}$, V.P. Lombardo ${ }^{4}$, R.E. Long ${ }^{71}$, L. Lopes ${ }^{124 a}$, D. Lopez Mateos ${ }^{57}$, J. Lorenz ${ }^{98}$, N. Lorenzo Martinez ${ }^{115}$, M. Losada ${ }^{162}$, P. Loscutoff ${ }^{14}$, F. Lo Sterzo ${ }^{132 a, 132 b}$, M.J. Losty ${ }^{159 a}$, X. Lou $^{40}$, A. Lounis ${ }^{115}$, K.F. Loureiro ${ }^{162}$, J. Love ${ }^{21}$, P.A. Love ${ }^{71}$, A.J. Lowe ${ }^{143, e}$, F. Lu ${ }^{32 a}$, H.J. Lubatti ${ }^{138}$, C. Luci ${ }^{132 a, 132 b}$, A. Lucotte $^{55}$, A. Ludwig ${ }^{43}$, D. Ludwig ${ }^{41}$, I. Ludwig ${ }^{48}$, J. Ludwig ${ }^{48}$, F. Luehring 60 , G. Luijckx ${ }^{105}$, W. $\operatorname{Lukas}^{61}$, D. Lumb ${ }^{48}$, L. Luminari ${ }^{132 a}$, E. Lund ${ }^{117}$, B. LundJensen $^{147}$, B. Lundberg ${ }^{79}$, J. Lundberg ${ }^{146 a, 146 b}$, O. Lundberg ${ }^{146 a, 146 b}$, J. Lundquist ${ }^{35}$, M. Lungwitz ${ }^{81}$, D. Lynn $^{24}$, E. Lytken ${ }^{79}$, H. Ma ${ }^{24}$, L.L. Ma ${ }^{173}$, J.A. Macana Goia ${ }^{93}$, G. Maccarrone ${ }^{47}$, A. Macchiolo ${ }^{99}$, B. Maček ${ }^{74}$, J. Machado Miguens ${ }^{124 a}$, R. Mackeprang ${ }^{35}$, R.J. Madaras ${ }^{14}$, W.F. Mader ${ }^{43}$, R. Maenner ${ }^{58 c}$, T. Maeno ${ }^{24}$, P. Mättig ${ }^{175}$, S. Mättig ${ }^{41}$, L. Magnoni ${ }^{29}$, E. Magradze ${ }^{54}$, K. Mahboubi ${ }^{48}$, S. Mahmoud ${ }^{73}$, G. Mahout ${ }^{17}$, C. Maiani ${ }^{136}$, C. Maidantchik ${ }^{23 a}$, A. Maio ${ }^{124 a, b}$, S. Majewski ${ }^{24}$, Y. Makida ${ }^{65}$, N. Makovec ${ }^{115}$, P. Mal ${ }^{136}$, B. Malaescu ${ }^{29}$, Pa. Malecki ${ }^{38}$, P. Malecki ${ }^{38}$, V.P. Maleev ${ }^{121}$, F. Malek ${ }^{55}$, U. Mallik ${ }^{62}$, D. Malon ${ }^{5}$, C. Malone ${ }^{143}$, S. Maltezos ${ }^{9}$, V. Malyshev ${ }^{107}$, S. Malyukov ${ }^{29}$, R. Mameghani ${ }^{98}$, J. Mamuzic ${ }^{12 b}$, A. Manabe ${ }^{65}$, L. Mandelli ${ }^{89 a}$, I. Mandić ${ }^{74}$, R. Mandrysch ${ }^{15}$, J. Maneira ${ }^{124 a}$, P.S. Mangeard ${ }^{88}$, L. Manhaes de Andrade Filho ${ }^{23 a}$, A. Mann ${ }^{54}$, P.M. Manning ${ }^{137}$, A. Manousakis-Katsikakis ${ }^{8}$, B. Mansoulie ${ }^{136}$, A. Mapelli ${ }^{29}$, L. Mapelli29 ${ }^{29}$ L. March ${ }^{80}$, J.F. Marchand ${ }^{28}$, F. Marchese ${ }^{133 a, 133 b}$, G. Marchiori ${ }^{78}$, M. Marcisovsky ${ }^{125}$, C.P. Marino ${ }^{169}$, F. Marroquim ${ }^{23 a}$, Z. Marshall ${ }^{29}$, F.K. Martens ${ }^{158}$, S. Marti-Garcia ${ }^{167}$, B. Martin ${ }^{29}$, B. Martin ${ }^{88}$, J.P. Martin ${ }^{93}$, T.A. Martin ${ }^{17}$, V.J. Martin ${ }^{45}$, B. Martin dit Latour ${ }^{49}$, S. Martin-Haugh ${ }^{149}$, M. Martinez ${ }^{11}$, V. Martinez Outschoorn ${ }^{57}$, A.C. Martyniuk ${ }^{169}$, M. Marx ${ }^{82}$, F. Marzano ${ }^{132 a}$, A. Marzin ${ }^{111}$, L. Masetti ${ }^{81}$, 
T. Mashimo ${ }^{155}$, R. Mashinistov ${ }^{94}$, J. Masik ${ }^{82}$, A.L. Maslennikov ${ }^{107}$, I. Massa ${ }^{19 a, 19 b}$, G. Massaro ${ }^{105}$, N. Massol ${ }^{4}$, A. Mastroberardino ${ }^{36 a, 36 b}$, T. Masubuchi ${ }^{155}$, P. Matricon ${ }^{115}$, H. Matsunaga ${ }^{155}$, T. Matsushita ${ }^{66}$, C. Mattravers ${ }^{118, c}$, J. Maurer ${ }^{83}$, S.J. Maxfield ${ }^{73}$, A. Mayne ${ }^{139}$, R. Mazini ${ }^{151}$, M. Mazur ${ }^{20}$, L. Mazzaferro ${ }^{133 a, 133 b}$, M. Mazzanti ${ }^{89 a}$, S.P. Mc Kee ${ }^{87}$, A. McCarn ${ }^{165}$, R.L. McCarthy ${ }^{148}$, T.G. McCarthy ${ }^{28}$, N.A. McCubbin ${ }^{129}$, K.W. McFarlane ${ }^{56}$, J.A. Mcfayden ${ }^{139}$, H. McGlone ${ }^{53}$, G. Mchedlidze $^{51 b}$, T. Mclaughlan ${ }^{17}$, S.J. McMahon ${ }^{129}$, R.A. McPherson ${ }^{169, k}$, A. Meade ${ }^{84}$, J. Mechnich ${ }^{105}$, M. Mechtel ${ }^{175}$, M. Medinnis ${ }^{41}$, R. Meera-Lebbai ${ }^{111}$, T. Meguro ${ }^{116}$, R. Mehdiyev ${ }^{93}$, S. Mehlhase ${ }^{35}$, A. Mehta ${ }^{73}$, K. Meier ${ }^{58 a}$, B. Meirose ${ }^{79}$, C. Melachrinos ${ }^{30}$, B.R. Mellado Garcia ${ }^{173}$, F. Meloni ${ }^{89 a, 89 b}$, L. Mendoza Navas ${ }^{162}$, Z. Meng ${ }^{151, u}$, A. Mengarelli ${ }^{19 a, 19 b}$, S. Menke ${ }^{99}$, E. Meoni ${ }^{161}$, K.M. Mercurio ${ }^{57}$, P. $\operatorname{Mermod}^{49}$, L. Merola102a,102b , C. Meroni ${ }^{89 a}$, F.S. Merritt ${ }^{30}$, H. Merritt ${ }^{109}$, A. Messina ${ }^{29, y}$, J. Metcalfe ${ }^{103}$, A.S. Mete ${ }^{163}$, C. Meyer ${ }^{81}$, C. Meyer $^{30}$, J-P. Meyer ${ }^{136}$, J. Meyer ${ }^{174}$, J. Meyer ${ }^{54}$, T.C. Meyer ${ }^{29}$, W.T. Meyer ${ }^{63}$,

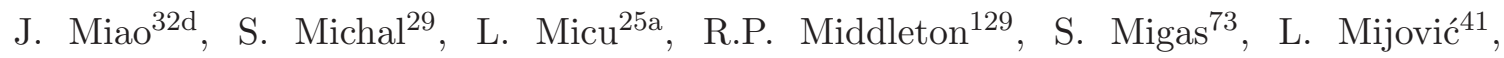
G. Mikenberg ${ }^{172}$, M. Mikestikova ${ }^{125}$, M. Mikuž ${ }^{74}$ D.W. Miller ${ }^{30}$, R.J. Miller ${ }^{88}$, W.J. Mills ${ }^{168}$, C. Mills ${ }^{57}$, A. Milov ${ }^{172}$, D.A. Milstead ${ }^{146 a, 146 b}$, D. Milstein ${ }^{172}$, A.A. Minaenko ${ }^{128}$, M. Miñano Moya ${ }^{167}$, I.A. Minashvili ${ }^{64}$, A.I. Mincer ${ }^{108}$, B. Mindur ${ }^{37}$, M. Mineev ${ }^{64}$, Y. Ming ${ }^{173}$, L.M. Mir ${ }^{11}$, G. Mirabelli ${ }^{132 a}$, J. Mitrevski ${ }^{137}$, V.A. Mitsou ${ }^{167}$, S. Mitsui ${ }^{65}$, P.S. Miyagawa ${ }^{139}$, K. Miyazaki ${ }^{66}$, J.U. Mjörnmark ${ }^{79}$, T. Moa ${ }^{146 a, 146 b}$, V. Moeller ${ }^{27}$, K. Mönig ${ }^{41}$, N. Möser ${ }^{20}$, S. Mohapatra ${ }^{148}$, W. Mohr ${ }^{48}$, R. MolesValls $^{167}$, J. Molina-Perez ${ }^{29}$, J. Monk ${ }^{77}$, E. Monnier ${ }^{83}$, J. Montejo Berlingen ${ }^{11}$, S. Montesano ${ }^{89 a, 89 b}$, F. Monticelli ${ }^{70}$, S. Monzani ${ }^{19 a, 19 b}$, R.W. Moore ${ }^{2}$, G.F. Moorhead ${ }^{86}$, C. Mora Herrera ${ }^{49}$, A. Moraes $^{53}$, N. Morange ${ }^{136}$, J. Morel ${ }^{54}$, G. Morello ${ }^{36 a, 36 b}$, D. Moreno ${ }^{81}$, M. Moreno Llácer ${ }^{167}$, P. Morettini ${ }^{50 a}$, M. Morgenstern ${ }^{43}$, M. Morii ${ }^{57}$, A.K. Morley ${ }^{29}$,

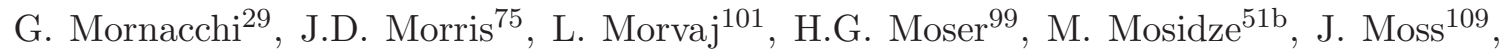
R. Mount ${ }^{143}$, E. Mountricha ${ }^{9, z}$, S.V. Mouraviev ${ }^{94}$, E.J.W. Moyse ${ }^{84}$, F. Mueller ${ }^{58 a}$, J. Mueller ${ }^{123}$, K. Mueller ${ }^{20}$, T.A. Müller ${ }^{98}$, T. Mueller ${ }^{81}$, D. Muenstermann ${ }^{29}$, Y. Munwes ${ }^{153}$, W.J. Murray ${ }^{129}$, I. Mussche ${ }^{105}$, E. Musto ${ }^{102 a, 102 b}$, A.G. Myagkov ${ }^{128}$, M. Myska ${ }^{125}$, J. Nadal ${ }^{11}$, K. Nagai ${ }^{160}$, K. Nagano ${ }^{65}$, A. Nagarkar ${ }^{109}$, Y. Nagasaka ${ }^{59}$, M. Nagel ${ }^{99}$, A.M. Nairz ${ }^{29}$, Y. Nakahama ${ }^{29}$, K. Nakamura ${ }^{155}$, T. Nakamura ${ }^{155}$,

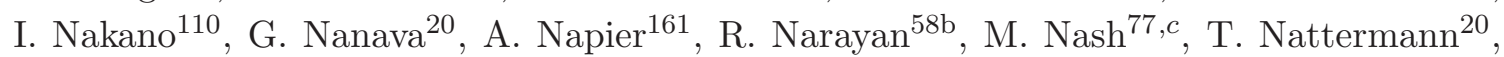
T. Naumann ${ }^{41}$, G. Navarro ${ }^{162}$, H.A. Neal ${ }^{87}$, P.Yu. Nechaeva ${ }^{94}$, T.J. Neep ${ }^{82}$, A. Negri ${ }^{119 a, 119 b}$, G. Negri ${ }^{29}$, S. Nektarijevic ${ }^{49}$, A. Nelson ${ }^{163}$, T.K. Nelson ${ }^{143}$, S. Nemecek ${ }^{125}$, P. Nemethy ${ }^{108}$, A.A. Nepomuceno ${ }^{23 a}$, M. Nessi2 ${ }^{29, a a}$, M.S. Neubauer ${ }^{165}$, A. Neusiedl ${ }^{81}$, R.M. Neves ${ }^{108}$, P. Nevski ${ }^{24}$, P.R. Newman ${ }^{17}$, V. Nguyen Thi Hong ${ }^{136}$, R.B. Nickerson ${ }^{118}$, R. Nicolaidou ${ }^{136}$, B. Nicquevert ${ }^{29}$, F. Niedercorn ${ }^{115}$, J. Nielsen ${ }^{137}$, N. Nikiforou ${ }^{34}$, A. Nikiforov ${ }^{15}$, V. Nikolaenko ${ }^{128}$, I. Nikolic-Audit ${ }^{78}$, K. Nikolics ${ }^{49}$, K. Nikolopoulos ${ }^{24}$, H. Nilsen ${ }^{48}$, P. Nilsson ${ }^{7}$, Y. Ninomiya ${ }^{155}$, A. Nisati ${ }^{132 a}$, T. Nishiyama ${ }^{66}$, R. Nisius ${ }^{99}$, L. Nodulman ${ }^{5}$, M. Nomachi ${ }^{116}$, I. Nomidis ${ }^{154}$, M. Nordberg ${ }^{29}$, P.R. Norton ${ }^{129}$, J. Novakova ${ }^{126}$, M. Nozaki ${ }^{65}$, L. Nozka ${ }^{113}$, I.M. Nugent ${ }^{159 a}$, A.E. Nuncio-Quiroz ${ }^{20}$, G. Nunes Hanninger ${ }^{86}$, T. Nunnemann ${ }^{98}$, E. Nurse ${ }^{77}$, B.J. O’Brien ${ }^{45}$, S.W. O'Neale ${ }^{17, *}$, D.C. O'Neil ${ }^{142}$, V. O'Shea ${ }^{53}$, L.B. Oakes ${ }^{98}$, F.G. Oakham ${ }^{28, d}$, H. Oberlack ${ }^{99}$, J. Ocariz ${ }^{78}$, A. Ochi ${ }^{66}$, S. Oda ${ }^{69}$, S. Odaka ${ }^{65}$, J. Odier ${ }^{83}$, H. Ogren ${ }^{60}$, 
A. $\mathrm{Oh}^{82}$, S.H. $\mathrm{Oh}^{44}$, C.C. $\mathrm{Ohm}^{146 a, 146 b}$, T. Ohshima ${ }^{101}$, S. Okada ${ }^{66}$, H. Okawa ${ }^{163}$, Y. Okumura ${ }^{101}$, T. Okuyama ${ }^{155}$, A. Olariu ${ }^{25 a}$, A.G. Olchevski ${ }^{64}$, S.A. Olivares Pino ${ }^{31 a}$, M. Oliveira ${ }^{124 a}, h$, D. Oliveira Damazio ${ }^{24}$, E. Oliver Garcia ${ }^{167}$, D. Olivito ${ }^{120}$, A. Olszewski $^{38}$, J. Olszowska ${ }^{38}$, A. Onofre ${ }^{124 a, a b}$, P.U.E. Onyisi ${ }^{30}$, C.J. Oram ${ }^{159 a}$, M.J. Oreglia ${ }^{30}$, Y. Oren ${ }^{153}$, D. Orestano ${ }^{134 a, 134 b}$, N. Orlando ${ }^{72 a, 72 b}$, I. Orlov ${ }^{107}$, C. Oropeza Barrera ${ }^{53}$, R.S. Orr ${ }^{158}$, B. Osculati ${ }^{50 a, 50 b}$, R. Ospanov ${ }^{120}$, C. Osuna ${ }^{11}$, G. Otero y Garzon ${ }^{26}$, J.P. Ottersbach ${ }^{105}$, M. Ouchrif ${ }^{135 d}$, E.A. Ouellette ${ }^{169}$, F. Ould-Saada ${ }^{117}$, A. Ouraou ${ }^{136}$, Q. Ouyang ${ }^{32 a}$, A. Ovcharova ${ }^{14}$, M. Owen ${ }^{82}$, S. Owen ${ }^{139}$, V.E. Ozcan ${ }^{18 a}$, N. Ozturk ${ }^{7}$, A. Pacheco Pages ${ }^{11}$, C. Padilla Aranda ${ }^{11}$, S. Pagan Griso ${ }^{14}$, E. Paganis ${ }^{139}$, F. Paige ${ }^{24}$, P. Pais ${ }^{84}$, K. Pajchel ${ }^{117}$, G. Palacino ${ }^{159 b}$, C.P. Paleari ${ }^{6}$, S. Palestini ${ }^{29}$, D. Pallin ${ }^{33}$, A. Palma ${ }^{124 a}$, J.D. Palmer ${ }^{17}$, Y.B. $\mathrm{Pan}^{173}$, E. Panagiotopoulou ${ }^{9}$, P. Pani ${ }^{105}$, N. Panikashvili ${ }^{87}$, S. Panitkin ${ }^{24}$, D. Pantea ${ }^{25 a}$, A. Papadelis ${ }^{146 a}$, Th.D. Papadopoulou ${ }^{9}$, A. Paramonov ${ }^{5}$, D. Paredes Hernandez ${ }^{33}$, W. Park ${ }^{24, a c}$, M.A. Parker ${ }^{27}$, F. Parodi ${ }^{50 a, 50 b}$, J.A. Parsons ${ }^{34}$, U. Parzefall ${ }^{48}$, S. Pashapour ${ }^{54}$, E. Pasqualucci ${ }^{132 a}$, S. Passaggio ${ }^{50 a}$, A. Passeri ${ }^{134 a}$, F. Pastore ${ }^{134 a, 134 b}$, Fr. Pastore ${ }^{76}$, G. Pásztor ${ }^{49, a d}$, S. Pataraia ${ }^{175}$, N. Patel ${ }^{150}$, J.R. Pater ${ }^{82}$, S. Patricelli ${ }^{102 a, 102 b}$, T. Pauly ${ }^{29}$, M. Pecsy ${ }^{144 a}$, M.I. Pedraza Morales ${ }^{173}$, S.V. Peleganchuk ${ }^{107}$, D. Pelikan ${ }^{166}$, H. Peng ${ }^{32 b}$, B. Penning ${ }^{30}$, A. Penson ${ }^{34}$, J. Penwell ${ }^{60}$, M. Perantoni ${ }^{23 a}$, K. Perez ${ }^{34, a e}$, T. Perez Cavalcanti ${ }^{41}$, E. Perez Codina ${ }^{159 a}$, M.T. Pérez García-Estañ ${ }^{167}$, V. Perez Reale ${ }^{34}$, L. Perini ${ }^{89 a, 89 b}$, H. Pernegger ${ }^{29}$, R. Perrino ${ }^{72 a}$, P. Perrodo ${ }^{4}$, V.D. Peshekhonov ${ }^{64}$, K. Peters ${ }^{29}$, B.A. Petersen ${ }^{29}$, J. Petersen ${ }^{29}$, T.C. Petersen ${ }^{35}$, E. Petit ${ }^{4}$, A. Petridis ${ }^{154}$, C. Petridou ${ }^{154}$, E. Petrolo ${ }^{132 a}$, F. Petrucci ${ }^{134 a, 134 b}$, D. Petschull ${ }^{41}$, M. Petteni ${ }^{142}$, R. Pezoa ${ }^{31 b}$, A. Phan $^{86}$, P.W. Phillips ${ }^{129}$, G. Piacquadio ${ }^{29}$, A. Picazio ${ }^{49}$, E. Piccaro ${ }^{75}$, M. Piccinini ${ }^{19 a}, 19 b$, S.M. $\operatorname{Piec}^{41}$, R. Piegaia ${ }^{26}$, D.T. Pignotti ${ }^{109}$, J.E. Pilcher ${ }^{30}$, A.D. Pilkington ${ }^{82}$, J. Pina ${ }^{124 a, b}$, M. Pinamonti ${ }^{164 a, 164 c}$, A. Pinder ${ }^{118}$, J.L. Pinfold ${ }^{2}$, B. Pinto ${ }^{124 a}$, C. Pizio ${ }^{89 a, 89 b}$, M. Plamondon ${ }^{169}$, M.-A. Pleier ${ }^{24}$, E. Plotnikova ${ }^{64}$, A. Poblaguev ${ }^{24}$, S. Poddar ${ }^{58 a}$, F. Podlyski ${ }^{33}$, L. Poggioli ${ }^{115}$, T. Poghosyan $^{20}$, M. Pohl ${ }^{49}$, F. Polci ${ }^{55}$, G. Polesello ${ }^{119 a}$, A. Policicchio ${ }^{36 a, 36 b}$, A. Polini ${ }^{19 a}$, J. Poll ${ }^{75}$, V. Polychronakos ${ }^{24}$, D. Pomeroy $^{22}$, K. Pommès ${ }^{29}$, L. Pontecorvo ${ }^{132 a}$, B.G. Pope ${ }^{88}$, G.A. Popeneciu ${ }^{25 a}$, D.S. Popovic ${ }^{12 a}$, A. Poppleton ${ }^{29}$, X. Portell Bueso ${ }^{29}$, G.E. Pospelov ${ }^{99}$, S. Pospisil ${ }^{127}$, I.N. Potrap ${ }^{99}$, C.J. Potter ${ }^{149}$, C.T. Potter ${ }^{114}$, G. Poulard ${ }^{29}$, J. Poveda ${ }^{60}$, V. Pozdnyakov ${ }^{64}$,

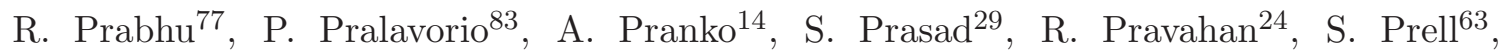
K. Pretzl $^{16}$, D. Price ${ }^{60}$, J. Price ${ }^{73}$, L.E. Price ${ }^{5}$, D. Prieur ${ }^{123}$, M. Primavera ${ }^{72 a}$, K. Prokofiev ${ }^{108}$, F. Prokoshin ${ }^{31 b}$, S. Protopopescu ${ }^{24}$, J. Proudfoot ${ }^{5}$, X. Prudent ${ }^{43}$, M. Przybycien ${ }^{37}$, H. Przysiezniak ${ }^{4}$, S. Psoroulas ${ }^{20}$, E. Ptacek ${ }^{114}$, E. Pueschel ${ }^{84}$, J. Purdham ${ }^{87}$, M. Purohit ${ }^{24, a c}$, P. Puzo ${ }^{115}$, Y. Pylypchenko ${ }^{62}$, J. Qian ${ }^{87}$, A. Quadt ${ }^{54}$,

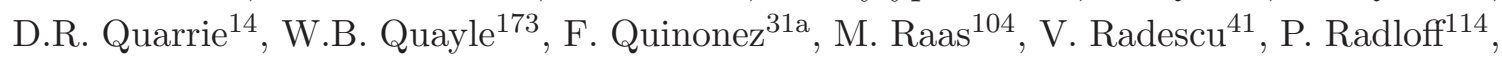
T. Rador ${ }^{18 a}$, F. Ragusa ${ }^{89 a, 89 b}$, G. Rahal ${ }^{178}$, A.M. Rahimi ${ }^{109}$, D. Rahm ${ }^{24}$, S. Rajagopalan $^{24}$, M. Rammensee ${ }^{48}$, M. Rammes ${ }^{141}$, A.S. Randle-Conde ${ }^{39}$, K. Randrianarivony ${ }^{28}$, F. Rauscher ${ }^{98}$, T.C. Rave ${ }^{48}$, M. Raymond ${ }^{29}$, A.L. Read ${ }^{117}$, D.M. Rebuzzi119a,119b, A. Redelbach ${ }^{174}$, G. Redlinger ${ }^{24}$, R. Reece ${ }^{120}$, K. Reeves ${ }^{40}$, E. Reinherz-Aronis ${ }^{153}$, A. Reinsch ${ }^{114}$, I. Reisinger ${ }^{42}$, C. Rembser ${ }^{29}$, Z.L. Ren ${ }^{151}$, A. Renaud ${ }^{115}$, M. Rescigno ${ }^{132 a}$, S. Resconi ${ }^{89 a}$, B. Resende ${ }^{136}$, P. Reznicek ${ }^{98}$, R. Rezvani ${ }^{158}$, R. Richter ${ }^{99}$, E. Richter- 
Was $^{4, a f}$, M. Ridel ${ }^{78}$, M. Rijpstra ${ }^{105}$, M. Rijssenbeek ${ }^{148}$, A. Rimoldi119a,119b, L. Rinaldi ${ }^{19 a}$, R.R. $\operatorname{Rios}^{39}$, I. Riu ${ }^{11}$, G. Rivoltella ${ }^{89 a, 89 b}$, F. Rizatdinova ${ }^{112}$, E. Rizvi ${ }^{75}$, S.H. Robertson ${ }^{85, k}$, A. Robichaud-Veronneau ${ }^{118}$, D. Robinson ${ }^{27}$, J.E.M. Robinson ${ }^{77}$, A. Robson ${ }^{53}$, J.G. Rocha de Lima ${ }^{106}$, C. Roda ${ }^{122 a, 122 b}$, D. Roda Dos Santos ${ }^{29}$, A. Roe ${ }^{54}$, S. Roe ${ }^{29}$, O. Røhne ${ }^{117}$, S. Rolli ${ }^{161}$, A. Romaniouk ${ }^{96}$, M. Romano ${ }^{19 a, 19 b}$, G. Romeo ${ }^{26}$, E. Romero Adam ${ }^{167}$, L. Roos ${ }^{78}$, E. Ros ${ }^{167}$, S. Rosati ${ }^{132 a}$, K. Rosbach ${ }^{49}$, A. $R{ }^{149}$, M. Rose ${ }^{76}$, G.A. Rosenbaum ${ }^{158}$, E.I. Rosenberg ${ }^{63}$, P.L. Rosendahl ${ }^{13}$,

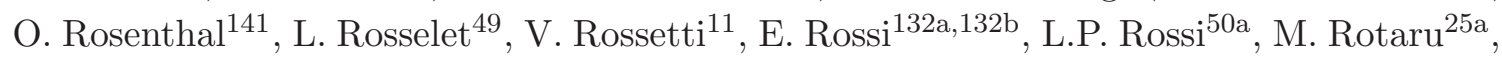
I. Roth ${ }^{172}$, J. Rothberg ${ }^{138}$, D. Rousseau ${ }^{115}$, C.R. Royon ${ }^{136}$, A. Rozanov ${ }^{83}$, Y. Rozen ${ }^{152}$, X. Ruan ${ }^{32 a, a g}$, F. Rubbo ${ }^{11}$, I. Rubinskiy ${ }^{41}$, B. Ruckert ${ }^{98}$, N. Ruckstuhl ${ }^{105}$, V.I. Rud ${ }^{97}$, C. Rudolph ${ }^{43}$, G. Rudolph ${ }^{61}$, F. Rühr ${ }^{6}$, F. Ruggieri ${ }^{134 a, 134 b}$, A. Ruiz-Martinez ${ }^{63}$, L. Rumyantsev ${ }^{64}$, Z. Rurikova ${ }^{48}$, N.A. Rusakovich ${ }^{64}$, J.P. Rutherfoord ${ }^{6}$, C. Ruwiedel ${ }^{14}$, P. Ruzicka ${ }^{125}$, Y.F. Ryabov ${ }^{121}$, P. Ryan ${ }^{88}$, M. Rybar ${ }^{126}$, G. Rybkin ${ }^{115}$, N.C. Ryder ${ }^{118}$, A.F. Saavedra ${ }^{150}$, I. Sadeh ${ }^{153}$, H.F-W. Sadrozinski ${ }^{137}$, R. Sadykov ${ }^{64}$, F. Safai Tehrani ${ }^{132 a}$, H. Sakamoto ${ }^{155}$, G. Salamanna ${ }^{75}$, A. Salamon ${ }^{133 a}$, M. Saleem ${ }^{111}$, D. Salek ${ }^{29}$, D. Salihagic ${ }^{99}$, A. Salnikov ${ }^{143}$, J. Salt ${ }^{167}$, B.M. Salvachua Ferrando ${ }^{5}$, D. Salvatore ${ }^{36 a, 36 b}$, F. Salvatore ${ }^{149}$, A. Salvucci ${ }^{104}$, A. Salzburger ${ }^{29}$, D. Sampsonidis ${ }^{154}$, B.H. Samset ${ }^{117}$, A. Sanchez ${ }^{102 a, 102 b}$, V. Sanchez Martinez ${ }^{167}$, H. Sandaker ${ }^{13}$, H.G. Sander ${ }^{81}$, M.P. Sanders ${ }^{98}$, M. Sandhoff ${ }^{175}$, T. Sandoval ${ }^{27}$, C. Sandoval ${ }^{162}$, R. Sandstroem ${ }^{99}$, D.P.C. Sankey ${ }^{129}$, A. Sansoni ${ }^{47}$, C. Santamarina Rios ${ }^{85}$, C. Santoni ${ }^{33}$, R. Santonico ${ }^{133 a, 133 b}$, H. Santos ${ }^{124 a}$, J.G. Saraiva ${ }^{124 a}$, T. Sarangi ${ }^{173}$, E. Sarkisyan-Grinbaum ${ }^{7}$, F. Sarri ${ }^{122 a, 122 b}$, G. Sartisohn ${ }^{175}$, O. Sasaki ${ }^{65}$, N. Sasao ${ }^{67}$, I. Satsounkevitch ${ }^{90}$, G. Sauvage ${ }^{4}$, E. Sauvan ${ }^{4}$, J.B. Sauvan ${ }^{115}$, P. Savard ${ }^{158, d}$, V. Savinov ${ }^{123}$, D.O. Savu ${ }^{29}$, L. Sawyer ${ }^{24, m}$, D.H. Saxon $^{53}$, J. Saxon ${ }^{120}$, C. Sbarra ${ }^{19 a}$, A. Sbrizzi ${ }^{19 a, 19 b}$, O. Scallon ${ }^{93}$, D.A. Scannicchio ${ }^{163}$, M. Scarcella ${ }^{150}$, J. Schaarschmidt ${ }^{115}$, P. Schacht ${ }^{99}$, D. Schaefer ${ }^{120}$, U. Schäfer ${ }^{81}$, S. Schaepe ${ }^{20}$, S. Schaetzel ${ }^{58 b}$, A.C. Schaffer ${ }^{115}$, D. Schaile ${ }^{98}$, R.D. Schamberger ${ }^{148}$, A.G. Schamov ${ }^{107}$, V. Scharf ${ }^{58 a}$, V.A. Schegelsky ${ }^{121}$, D. Scheirich ${ }^{87}$, M. Schernau ${ }^{163}$, M.I. Scherzer ${ }^{34}$, C. Schiavi ${ }^{50 a, 50 b}$, J. Schieck ${ }^{98}$, M. Schioppa ${ }^{36 a, 36 b}$, S. Schlenker ${ }^{29}$, E. Schmidt ${ }^{48}$, K. Schmieden ${ }^{20}$, C. Schmitt ${ }^{81}$, S. Schmitt ${ }^{58 b}$, M. Schmitz ${ }^{20}$, B. Schneider ${ }^{16}$, U. Schnoor ${ }^{43}$, A. Schöning ${ }^{58 b}$, A.L.S. Schorlemmer ${ }^{54}$, M. Schott ${ }^{29}$, D. Schouten ${ }^{159 a}$, J. Schovancova ${ }^{125}$, M. Schram ${ }^{85}$, C. Schroeder ${ }^{81}$, N. Schroer ${ }^{58 c}$, M.J. Schultens ${ }^{20}$, J. Schultes ${ }^{175}$, H.-C. Schultz-Coulon ${ }^{58 a}$, H. Schulz ${ }^{15}$, J.W. Schumacher ${ }^{20}$, M. Schumacher ${ }^{48}$, B.A. Schumm ${ }^{137}$, Ph. Schune ${ }^{136}$, C. Schwanenberger ${ }^{82}$, A. Schwartzman ${ }^{143}$, Ph. Schwemling ${ }^{78}$, R. Schwienhorst ${ }^{88}$, R. Schwierz ${ }^{43}$, J. Schwindling ${ }^{136}$, T. Schwindt ${ }^{20}$, M. Schwoerer ${ }^{4}$, G. Sciolla ${ }^{22}$, W.G. Scott ${ }^{129}$, J. Searcy ${ }^{114}$, G. Sedov ${ }^{41}$, E. Sedykh ${ }^{121}$, S.C. Seidel ${ }^{103}$, A. Seiden ${ }^{137}$, F. Seifert ${ }^{43}$, J.M. Seixas ${ }^{23 a}$, G. Sekhniaidze ${ }^{102 a}$, S.J. Sekula ${ }^{39}$, K.E. Selbach ${ }^{45}$, D.M. Seliverstov ${ }^{121}$, B. Sellden ${ }^{146 a}$, G. Sellers ${ }^{73}$, M. Seman ${ }^{14 b}$, N. Semprini-Cesari ${ }^{19 a, 19 b}$, C. Serfon ${ }^{98}$, L. Serin ${ }^{115}$, L. Serkin ${ }^{54}$, R. Seuster ${ }^{99}$, H. Severini ${ }^{111}$, A. Sfyrla ${ }^{29}$, E. Shabalina ${ }^{54}$, M. Shamim ${ }^{114}$, L.Y. Shan ${ }^{32 a}$, J.T. Shank ${ }^{21}$, Q.T. Shao ${ }^{86}$, M. Shapiro ${ }^{14}$, P.B. Shatalov ${ }^{95}$, K. Shaw ${ }^{164 a, 164 c}$, D. Sherman ${ }^{176}$, P. Sherwood ${ }^{77}$, A. Shibata ${ }^{108}$, H. Shichi ${ }^{101}$, S. Shimizu ${ }^{29}$, M. Shimojima ${ }^{100}$, T. Shin ${ }^{56}$, M. Shiyakova ${ }^{64}$, A. Shmeleva ${ }^{94}$, M.J. Shochet ${ }^{30}$, D. Short ${ }^{118}$, S. Shrestha ${ }^{63}$, E. Shulga ${ }^{96}$, M.A. Shupe ${ }^{6}$, P. Sicho ${ }^{125}$, A. Sidoti ${ }^{132 a}$, F. Siegert ${ }^{48}$, Dj. Sijacki ${ }^{12 a}$, O. Silbert ${ }^{172}$, J. Silva ${ }^{124 a}$, Y. Silver ${ }^{153}$, 
D. Silverstein ${ }^{143}$, S.B. Silverstein ${ }^{146 a}$, V. Simak $^{127}$, O. Simard ${ }^{136}$, Lj. Simic $^{12 a}$, S. Simion ${ }^{115}$, E. Simioni ${ }^{81}$, B. Simmons ${ }^{77}$, R. Simoniello ${ }^{89 a, 89 b}$, M. Simonyan ${ }^{35}$, P. Sinervo ${ }^{158}$, N.B. Sinev ${ }^{114}$, V. Sipica ${ }^{141}$, G. Siragusa ${ }^{174}$, A. Sircar ${ }^{24}$, A.N. Sisakyan ${ }^{64}$, S.Yu. Sivoklokov ${ }^{97}$, J. Sjölin ${ }^{146 a, 146 b}$, T.B. Sjursen ${ }^{13}$, L.A. Skinnari ${ }^{14}$, H.P. Skottowe ${ }^{57}$, K. Skovpen ${ }^{107}$, P. Skubic ${ }^{111}$, M. Slater ${ }^{17}$, T. Slavicek ${ }^{127}$, K. Sliwa ${ }^{161}$, V. Smakhtin ${ }^{172}$, B.H. Smart $^{45}$, S.Yu. Smirnov ${ }^{96}$, Y. Smirnov $^{96}$, L.N. Smirnova ${ }^{97}$, O. Smirnova ${ }^{79}$,

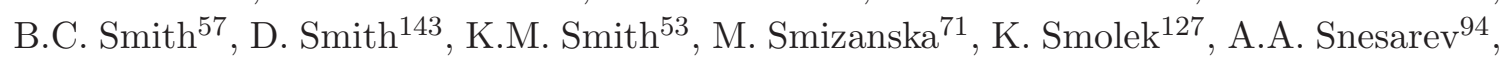
S.W. Snow ${ }^{82}$, J. Snow ${ }^{111}$, S. Snyder ${ }^{24}$, R. Sobie ${ }^{169, k}$, J. Sodomka ${ }^{127}$, A. Soffer ${ }^{153}$, C.A. Solans ${ }^{167}$, M. Solar ${ }^{127}$, J. Solc ${ }^{127}$, E.Yu. Soldatov ${ }^{96}$, U. Soldevila ${ }^{167}$, E. Solfaroli Camillocci ${ }^{132 a, 132 b}$, A.A. Solodkov ${ }^{128}$, O.V. Solovyanov ${ }^{128}$, N. Soni ${ }^{2}$, V. Sopko ${ }^{127}$, B. Sopko ${ }^{127}$, M. Sosebee ${ }^{7}$, R. Soualah ${ }^{164 a, 164 c}$, A. Soukharev ${ }^{107}$, S. Spagnolo ${ }^{72 a, 72 b}$, F. Spanò ${ }^{76}$, R. Spighi ${ }^{19 a}$, G. Spigo ${ }^{29}$, F. Spila ${ }^{132 a, 132 b}$, R. Spiwoks ${ }^{29}$, M. Spousta ${ }^{126}$, T. Spreitzer ${ }^{158}$, B. Spurlock ${ }^{7}$, R.D. St. Denis ${ }^{53}$, J. Stahlman ${ }^{120}$, R. Stamen ${ }^{58 a}$, E. Stanecka ${ }^{38}$, R.W. Stanek ${ }^{5}$, C. Stanescu ${ }^{134 a}$, M. Stanescu-Bellu ${ }^{41}$, S. Stapnes ${ }^{117}$, E.A. Starchenko ${ }^{128}$, J. Stark ${ }^{55}$, P. Staroba ${ }^{125}$, P. Starovoitov ${ }^{41}$, A. Staude ${ }^{98}$, P. Stavina ${ }^{144 a}$, G. Steele ${ }^{53}$, P. Steinbach ${ }^{43}$, P. Steinberg ${ }^{24}$, I. Stekl ${ }^{127}$, B. Stelzer ${ }^{142}$, H.J. Stelzer ${ }^{88}$, O. Stelzer-Chilton ${ }^{159 a}$, H. Stenzel ${ }^{52}$, S. Stern ${ }^{99}$, G.A. Stewart ${ }^{29}$, J.A. Stillings ${ }^{20}$, M.C. Stockton ${ }^{85}$, K. Stoerig ${ }^{48}$, G. Stoicea ${ }^{25 a}$, S. Stonjek ${ }^{99}$, P. Strachota ${ }^{126}$, A.R. Stradling ${ }^{7}$, A. Straessner ${ }^{43}$, J. Strandberg ${ }^{147}$, S. Strandberg ${ }^{146 a, 146 b}$, A. Strandlie ${ }^{117}$, M. Strang ${ }^{109}$, E. Strauss ${ }^{143}$, M. Strauss ${ }^{111}$, P. Strizenec ${ }^{144 b}$, R. Ströhmer ${ }^{174}$, D.M. Strom ${ }^{114}$, J.A. Strong ${ }^{76, *}$, R. Stroynowski ${ }^{39}$, J. Strube ${ }^{129}$, B. Stugu ${ }^{13}$, I. Stumer ${ }^{24, *}$, J. Stupak ${ }^{148}$, P. Sturm ${ }^{175}$, N.A. Styles ${ }^{41}$, D.A. Soh ${ }^{151, w}$, D. Su ${ }^{143}$, HS. Subramania ${ }^{2}$, A. Succurro ${ }^{11}$, Y. Sugaya ${ }^{116}$, C. Suhr ${ }^{106}$, K. Suita ${ }^{66}$, M. Suk ${ }^{126}$, V.V. Sulin ${ }^{94}$, S. Sultansoyd ${ }^{3 d}$, T. Sumida ${ }^{67}$, X. Sun ${ }^{55}$, J.E. Sundermann ${ }^{48}$, K. Suruliz ${ }^{139}$, G. Susinno ${ }^{36 a, 36 b}$, M.R. Sutton ${ }^{149}$, Y. Suzuki ${ }^{65}$, Y. Suzuki ${ }^{66}$, M. Svatos ${ }^{125}$, S. Swedish ${ }^{168}$, I. Sykora ${ }^{144 a}$, T. Sykora ${ }^{126}$, J. Sánchez ${ }^{167}$, D. Ta ${ }^{105}$, K. Tackmann ${ }^{41}$, A. Taffard ${ }^{163}$, R. Tafirout ${ }^{159 a}$, N. Taiblum ${ }^{153}$, Y. Takahashi ${ }^{101}$, H. Takai ${ }^{24}$, R. Takashima ${ }^{68}$, H. Takeda ${ }^{66}$, T. Takeshita $^{140}$, Y. Takubo ${ }^{65}$, M. Talby ${ }^{83}$, A. Talyshev ${ }^{107, f}$, M.C. Tamsett ${ }^{24}$, J. Tanaka ${ }^{155}$, R. Tanaka ${ }^{115}$, S. Tanaka ${ }^{131}$, S. Tanaka ${ }^{65}$, A.J. Tanasijczuk ${ }^{142}$, K. Tani ${ }^{66}$, N. Tannoury ${ }^{83}$, S. Tapprogge ${ }^{81}$, D. Tardif ${ }^{158}$, S. Tarem ${ }^{152}$, F. Tarrade ${ }^{28}$, G.F. Tartarelli ${ }^{89 a}$, P. Tas ${ }^{126}$, M. Tasevsky ${ }^{125}$, E. Tassi ${ }^{36 a, 36 b}$, M. Tatarkhanov ${ }^{14}$, Y. Tayalati ${ }^{135 d}$, C. Taylor ${ }^{77}$, F.E. Taylor ${ }^{92}$, G.N. Taylor ${ }^{86}$, W. Taylor ${ }^{159 b}$, M. Teinturier ${ }^{115}$, M. Teixeira Dias Castanheira ${ }^{75}$, P. Teixeira$\operatorname{Dias}^{76}$, K.K. Temming ${ }^{48}$, H. Ten Kate ${ }^{29}$, P.K. Teng ${ }^{151}$, S. Terada ${ }^{65}$, K. Terashi ${ }^{155}$, J. Terron ${ }^{80}$, M. Testa ${ }^{47}$, R.J. Teuscher ${ }^{158, k}$, J. Therhaag ${ }^{20}$, T. Theveneaux-Pelzer ${ }^{78}$, S. Thoma ${ }^{48}$, J.P. Thomas ${ }^{17}$, E.N. Thompson ${ }^{34}$, P.D. Thompson ${ }^{17}$, P.D. Thompson ${ }^{158}$, A.S. Thompson ${ }^{53}$, L.A. Thomsen ${ }^{35}$, E. Thomson ${ }^{120}$, M. Thomson ${ }^{27}$, R.P. Thun ${ }^{87}$, F. $\operatorname{Tian}^{34}$, M.J. Tibbetts ${ }^{14}$, T. Tic ${ }^{125}$, V.O. Tikhomirov ${ }^{94}$, Y.A. Tikhonov ${ }^{107, f}$, S. Timoshenko ${ }^{96}$, P. Tipton ${ }^{176}$, F.J. Tique Aires Viegas ${ }^{29}$, S. Tisserant ${ }^{83}$, T. Todorov ${ }^{4}$, S. Todorova-Nova ${ }^{161}$, B. Toggerson ${ }^{163}$, J. Tojo ${ }^{69}$, S. Tokár ${ }^{144 a}$, K. Tokunaga ${ }^{66}$,

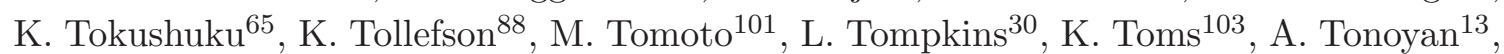
C. Topfel ${ }^{16}$, N.D. Topilin ${ }^{64}$, I. Torchiani ${ }^{29}$, E. Torrence ${ }^{114}$, H. Torres ${ }^{78}$, E. Torró Pastor $^{167}$, J. Toth ${ }^{83, a d}$, F. Touchard ${ }^{83}$, D.R. Tovey ${ }^{139}$, T. Trefzger ${ }^{174}$, L. Tremblet ${ }^{29}$, A. Tricoli ${ }^{29}$, I.M. Trigger ${ }^{159 a}$, S. Trincaz-Duvoid ${ }^{78}$, M.F. Tripiana ${ }^{70}$, W. Trischuk ${ }^{158}$, 
B. Trocmé ${ }^{55}$, C. Troncon ${ }^{89 a}$, M. Trottier-McDonald ${ }^{142}$, M. Trzebinski ${ }^{38}$, A. Trzupek ${ }^{38}$, C. Tsarouchas $^{29}$, J.C-L. Tseng ${ }^{118}$, M. Tsiakiris ${ }^{105}$, P.V. Tsiareshka ${ }^{90}$, D. Tsionou ${ }^{4, a h}$, G. Tsipolitis ${ }^{9}$, V. Tsiskaridze ${ }^{48}$, E.G. Tskhadadze ${ }^{51 a}$, I.I. Tsukerman ${ }^{95}$, V. Tsulaia ${ }^{14}$, J.W. Tsung ${ }^{20}$, S. Tsuno ${ }^{65}$, D. Tsybychev ${ }^{148}$, A. Tua ${ }^{139}$, A. Tudorache ${ }^{25 a}$, V. Tudorache ${ }^{25 a}$, J.M. Tuggle ${ }^{30}$, M. Turala ${ }^{38}$, D. Turecek ${ }^{127}$, I. Turk Cakir $^{3 e}$, E. Turlay ${ }^{105}$, R. Turra ${ }^{89 a, 89 b}$, P.M. Tuts ${ }^{34}$, A. Tykhonov ${ }^{74}$, M. Tylmad ${ }^{146 a, 146 b}$, M. Tyndel ${ }^{129}$, G. Tzanakos ${ }^{8}$, K. Uchida ${ }^{20}$, I. Ueda ${ }^{155}$, R. Ueno ${ }^{28}$, M. Ugland ${ }^{13}, \mathrm{M}$. Uhlenbrock ${ }^{20}, \mathrm{M}$. Uhrmacher ${ }^{54}$,

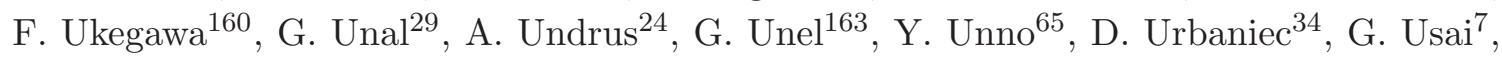

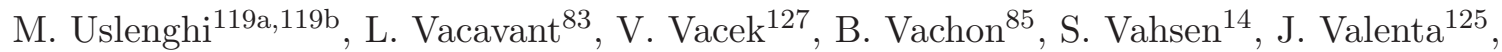
P. Valente ${ }^{132 a}$, S. Valentinetti ${ }^{19 a, 19 b}$, S. Valkar ${ }^{126}$, E. Valladolid Gallego ${ }^{167}$, S. Vallecorsa ${ }^{152}$, J.A. Valls Ferrer ${ }^{167}$, H. van der Graaf ${ }^{105}$, E. van der Kraaij ${ }^{105}$, R. Van Der Leeuw ${ }^{105}$, E. van der Poel ${ }^{105}$, D. van der $\operatorname{Ster}^{29}$, N. van $\operatorname{Eldik}^{29}$, P. van Gemmeren ${ }^{5}$, I. van Vulpen ${ }^{105}$,

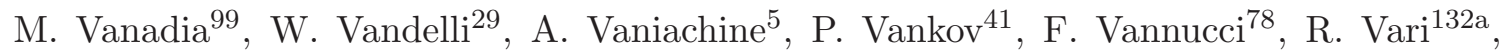
T. Varol ${ }^{84}$, D. Varouchas ${ }^{14}$, A. Vartapetian ${ }^{7}$, K.E. Varvell ${ }^{150}$, V.I. Vassilakopoulos ${ }^{56}$, F. Vazeille ${ }^{33}$, T. Vazquez Schroeder ${ }^{54}$, G. Vegni ${ }^{89 a, 89 b}$, J.J. Veillet ${ }^{115}$, F. Veloso ${ }^{124 a}$, R. Veness ${ }^{29}$, S. Veneziano ${ }^{132 a}$, A. Ventura ${ }^{72 a, 72 b}$, D. Ventura ${ }^{84}$, M. Venturi ${ }^{48}$, N. Venturi ${ }^{158}$, V. Vercesi ${ }^{119 a}$, M. Verducci ${ }^{138}$, W. Verkerke ${ }^{105}$, J.C. Vermeulen ${ }^{105}$, A. Vest ${ }^{43}$, M.C. Vetterli ${ }^{142, d}$, I. Vichou ${ }^{165}$, T. Vickey ${ }^{145 b, a i}$, O.E. Vickey Boeriu ${ }^{145 b}$, G.H.A. Viehhauser ${ }^{118}$, S. Viel ${ }^{168}$, M. Villa ${ }^{19 a, 19 b}$, M. Villaplana Perez ${ }^{167}$, E. Vilucchi ${ }^{47}$, M.G. Vincter ${ }^{28}$, E. $\operatorname{Vinek}^{29}$, V.B. Vinogradov ${ }^{64}$, M. Virchaux ${ }^{136, *}$, J. Virzi ${ }^{14}$, O. Vitells ${ }^{172}$, M. Viti ${ }^{41}$, I. Vivarelli ${ }^{48}$, F. Vives Vaque $^{2}$, S. Vlachos ${ }^{9}$, D. Vladoiu ${ }^{98}$, M. Vlasak ${ }^{127}$, A. $\operatorname{Vogel}^{20}$, P. Vokac ${ }^{127}$, G. Volpi ${ }^{47}$, M. Volpi ${ }^{86}$, G. Volpini ${ }^{89 a}$, H. von der Schmitt $^{99}$, J. von Loeben ${ }^{99}, H$. von Radziewski ${ }^{48}$, E. von Toerne ${ }^{20}$, V. Vorobel ${ }^{126}$, V. Vorwerk ${ }^{11}$, M. $\operatorname{Vos}^{167}$, R. Voss ${ }^{29}$, T.T. Voss ${ }^{175}$, J.H. Vossebeld ${ }^{73}$, N. Vranjes ${ }^{136}$, M. Vranjes Milosavljevic ${ }^{105}, \mathrm{~V} . \mathrm{Vrba}^{125}, \mathrm{M}$. Vreeswijk ${ }^{105}, \mathrm{~T} . \mathrm{Vu} \mathrm{Anh}^{48}$, R. Vuillermet ${ }^{29}$, I. Vukotic ${ }^{115}$, W. Wagner ${ }^{175}$, P. Wagner ${ }^{120}$, H. Wahlen ${ }^{175}$, S. Wahrmund ${ }^{43}$, J. Wakabayashi ${ }^{101}$, S. Walch ${ }^{87}$, J. Walder ${ }^{71}$, R. Walker ${ }^{98}$, W. Walkowiak ${ }^{141}$, R. Wall ${ }^{176}$, P. Waller ${ }^{73}$, C. Wang ${ }^{44}$, H. Wang ${ }^{173}$, H. Wang ${ }^{32 b, a j}$, J. Wang ${ }^{151}$, J. Wang ${ }^{55}$, R. Wang ${ }^{103}$, S.M. Wang ${ }^{151}$, T. Wang ${ }^{20}$, A. Warburton ${ }^{85}$, C.P. Ward ${ }^{27}$, M. Warsinsky ${ }^{48}$, A. Washbrook ${ }^{45}$, C. Wasicki ${ }^{41}$, P.M. Watkins ${ }^{17}$, A.T. Watson ${ }^{17}$, I.J. Watson ${ }^{150}$, M.F. Watson ${ }^{17}$, G. Watts ${ }^{138}$, S. Watts ${ }^{82}$, A.T. Waugh ${ }^{150}$, B.M. Waugh ${ }^{77}$, M. Weber ${ }^{129}$, M.S. Weber ${ }^{16}$, P. Weber ${ }^{54}$, A.R. Weidberg ${ }^{118}$, P. Weigell ${ }^{99}$, J. Weingarten ${ }^{54}$, C. Weiser ${ }^{48}$, H. Wellenstein ${ }^{22}$, P.S. Wells ${ }^{29}$, T. Wenaus ${ }^{24}$, D. Wendland ${ }^{15}$, Z. Weng ${ }^{151, w}$, T. Wengler ${ }^{29}$,

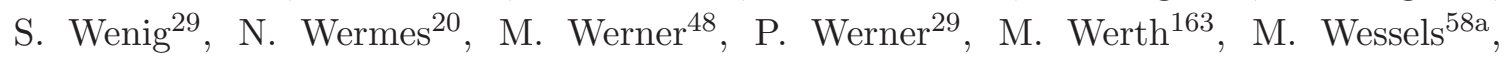
J. Wetter ${ }^{161}$, C. Weydert ${ }^{55}$, K. Whalen ${ }^{28}$, S.J. Wheeler-Ellis ${ }^{163}$, A. White ${ }^{7}$, M.J. White ${ }^{86}$, S. White ${ }^{122 a, 122 b}$, S.R. Whitehead ${ }^{118}$, D. Whiteson ${ }^{163}$, D. Whittington ${ }^{60}$, F. Wicek ${ }^{115}$, D. Wicke ${ }^{175}$, F.J. Wickens ${ }^{129}$, W. Wiedenmann ${ }^{173}$, M. Wielers ${ }^{129}$, P. Wienemann ${ }^{20}$, C. Wiglesworth ${ }^{75}$, L.A.M. Wiik-Fuchs ${ }^{48}$, P.A. Wijeratne ${ }^{77}$, A. Wildauer ${ }^{167}$, M.A. Wildt ${ }^{41, s}$, I. Wilhelm ${ }^{126}$, H.G. Wilkens ${ }^{29}$, J.Z. Will ${ }^{98}$, E. Williams ${ }^{34}$, H.H. Williams ${ }^{120}$, W. Willis ${ }^{34}$, S. Willocq ${ }^{84}$, J.A. Wilson ${ }^{17}$, M.G. Wilson ${ }^{143}$, A. Wilson ${ }^{87}$, I. Wingerter-Seez ${ }^{4}$, S. Winkelmann ${ }^{48}$, F. Winklmeier ${ }^{29}$, M. Wittgen ${ }^{143}$, M.W. Wolter ${ }^{38}$, H. Wolters ${ }^{124 a, h}$, W.C. Wong ${ }^{40}$, G. Wooden ${ }^{87}$, B.K. Wosiek ${ }^{38}$, J. Wotschack ${ }^{29}$, M.J. Woudstra ${ }^{82}$, K.W. Wozniak ${ }^{38}$, K. Wraight ${ }^{53}$, C. Wright ${ }^{53}$, M. Wright ${ }^{53}$, B. Wrona ${ }^{73}$, S.L. Wu ${ }^{173}$, 
X. Wu ${ }^{49}$, Y. Wu ${ }^{32 b, a k}$, E. Wulf ${ }^{34}$, B.M. Wynne ${ }^{45}$, S. Xella ${ }^{35}$, M. Xiao ${ }^{136}$, S. Xie ${ }^{48}$, C. $\mathrm{Xu}^{32 \mathrm{~b}, z}, \mathrm{D} \cdot \mathrm{Xu}^{139}$, B. Yabsley ${ }^{150}$, S. Yacoob ${ }^{145 b}$, M. Yamada ${ }^{65}$, H. Yamaguchi $^{155}$, A. Yamamoto ${ }^{65}$, K. Yamamoto ${ }^{63}$, S. Yamamoto ${ }^{155}$, T. Yamamura ${ }^{155}$, T. Yamanaka ${ }^{155}$, J. Yamaoka ${ }^{44}$, T. Yamazaki ${ }^{155}$, Y. Yamazaki ${ }^{66}$, Z. Yan ${ }^{21}$, H. Yang ${ }^{87}$, U.K. Yang ${ }^{82}$, Y. Yang ${ }^{60}$, Z. Yang ${ }^{146 a, 146 b}$, S. Yanush ${ }^{91}$, L. Yao ${ }^{32 a}$, Y. Yao ${ }^{14}$, Y. Yasu ${ }^{65}$, G.V. Ybeles Smit $^{130}$, J. Ye ${ }^{39}$, S. $\mathrm{Ye}^{24}$, M. Yilmaz ${ }^{3 \mathrm{c}}$, R. Yoosoofmiya ${ }^{123}$, K. Yorita ${ }^{171}$, R. Yoshida ${ }^{5}$, C. Young ${ }^{143}$, C.J. Young ${ }^{118}$, S. Youssef ${ }^{21}$, D. Yu ${ }^{24}$, J. Yu ${ }^{7}$, J. Yu ${ }^{112}$, L. $Y{ }^{66}{ }^{66}$ A. Yurkewicz ${ }^{106}$, B. Zabinski ${ }^{38}$, R. Zaidan ${ }^{62}$, A.M. Zaitsev ${ }^{128}$, Z. Zajacova ${ }^{29}$, L. Zanello132a,132b , A. Zaytsev ${ }^{107}$, C. Zeitnitz ${ }^{175}$, M. Zeman ${ }^{125}$, A. Zemla ${ }^{38}$, C. Zendler ${ }^{20}$, O. Zenin ${ }^{128}$, T. Ženišs ${ }^{144 a}$, Z. Zinonos ${ }^{122 a, 122 b}$, S. Zenz ${ }^{14}$, D. Zerwas ${ }^{115}$, G. Zevi della Porta ${ }^{57}$, Z. Zhan ${ }^{32 \mathrm{~d}}$, D. Zhang ${ }^{32 \mathrm{~b}, a j}$, H. Zhang ${ }^{88}$, J. Zhang ${ }^{5}$, X. Zhang ${ }^{32 \mathrm{~d}}$, Z. Zhang ${ }^{115}$, L. Zhao ${ }^{108}$, T. Zhao ${ }^{138}$, Z. Zhao ${ }^{32 b}$, A. Zhemchugov ${ }^{64}$, J. Zhong ${ }^{118}$, B. Zhou ${ }^{87}$, N. Zhou ${ }^{163}$, Y. Zhou ${ }^{151}$, C.G. Zhu ${ }^{32 \mathrm{~d}}, \mathrm{H} . \mathrm{Zhu}^{41}$, J. Zhu ${ }^{87}$, Y. Zhu ${ }^{32 \mathrm{~b}}$, X. Zhuang ${ }^{98}$, V. Zhuravlov ${ }^{99}$, D. Zieminska ${ }^{60}$, N.I. Zimin ${ }^{64}$, R. Zimmermann ${ }^{20}$, S. Zimmermann ${ }^{20}$, S. Zimmermann ${ }^{48}$, M. Ziolkowski ${ }^{141}$, R. Zitoun ${ }^{4}$, L. Živković ${ }^{34}$, V.V. Zmouchko ${ }^{128, *}$, G. Zobernig ${ }^{173}$, A. Zoccoli ${ }^{19 a, 19 b}$, M. zur Nedden ${ }^{15}$, V. Zutshi ${ }^{106}$ and L. Zwalinski ${ }^{29}$.

1: University at Albany, Albany NY, United States of America

2: Department of Physics, University of Alberta, Edmonton AB, Canada

${ }^{3}$ : (a) Department of Physics, Ankara University, Ankara; ${ }^{(b)}$ Department of Physics, Dumlupinar University, Kutahya; ${ }^{(c)}$ Department of Physics, Gazi University, Ankara; ${ }^{(d)}$ Division of Physics, TOBB University of Economics and Technology, Ankara; ${ }^{(e)}$ Turkish Atomic Energy Authority, Ankara, Turkey

4: LAPP, CNRS/IN2P3 and Université de Savoie, Annecy-le-Vieux, France

5: High Energy Physics Division, Argonne National Laboratory, Argonne IL, United States of America

6: Department of Physics, University of Arizona, Tucson AZ, United States of America

7: Department of Physics, The University of Texas at Arlington, Arlington TX, United States of America

8: Physics Department, University of Athens, Athens, Greece

9: Physics Department, National Technical University of Athens, Zografou, Greece

10: Institute of Physics, Azerbaijan Academy of Sciences, Baku, Azerbaijan

11: Institut de Física d'Altes Energies and Departament de Física de la Universitat Autònoma de Barcelona and ICREA, Barcelona, Spain

12: ${ }^{(a)}$ Institute of Physics, University of Belgrade, Belgrade; ${ }^{(b)}$ Vinca Institute of Nuclear Sciences, University of Belgrade, Belgrade, Serbia

13: Department for Physics and Technology, University of Bergen, Bergen, Norway

14: Physics Division, Lawrence Berkeley National Laboratory and University of California, Berkeley CA, United States of America

15: Department of Physics, Humboldt University, Berlin, Germany

16: Albert Einstein Center for Fundamental Physics and Laboratory for High Energy Physics, University of Bern, Bern, Switzerland 
17: School of Physics and Astronomy, University of Birmingham, Birmingham, United Kingdom

18: ${ }^{(a)}$ Department of Physics, Bogazici University, Istanbul; ${ }^{(b)}$ Division of Physics, Dogus University, Istanbul; ${ }^{(c)}$ Department of Physics Engineering, Gaziantep University, Gaziantep; ${ }^{(d)}$ Department of Physics, Istanbul Technical University, Istanbul, Turkey

19: ${ }^{(a)}$ INFN Sezione di Bologna; ${ }^{(b)}$ Dipartimento di Fisica, Università di Bologna, Bologna, Italy

20: Physikalisches Institut, University of Bonn, Bonn, Germany

21: Department of Physics, Boston University, Boston MA, United States of America

22: Department of Physics, Brandeis University, Waltham MA, United States of America

23: ${ }^{(a)}$ Universidade Federal do Rio De Janeiro COPPE/EE/IF, Rio de Janeiro; ${ }^{(b)}$ Federal University of Juiz de Fora (UFJF), Juiz de Fora; ${ }^{(c)}$ Federal University of Sao Joao del Rei (UFSJ), Sao Joao del Rei; ${ }^{(d)}$ Instituto de Fisica, Universidade de Sao Paulo, Sao Paulo, Brazil

24: Physics Department, Brookhaven National Laboratory, Upton NY, United States of America

25: ${ }^{(a)}$ National Institute of Physics and Nuclear Engineering, Bucharest; ${ }^{(b)}$ University Politehnica Bucharest, Bucharest; ${ }^{(c)}$ West University in Timisoara, Timisoara, Romania

26: Departamento de Física, Universidad de Buenos Aires, Buenos Aires, Argentina

27: Cavendish Laboratory, University of Cambridge, Cambridge, United Kingdom

28: Department of Physics, Carleton University, Ottawa ON, Canada

29: CERN, Geneva, Switzerland

30: Enrico Fermi Institute, University of Chicago, Chicago IL, United States of America

31: ${ }^{(a)}$ Departamento de Fisica, Pontificia Universidad Católica de Chile, Santiago;

${ }^{(b)}$ Departamento de Física, Universidad Técnica Federico Santa María, Valparaíso, Chile

32: ${ }^{(a)}$ Institute of High Energy Physics, Chinese Academy of Sciences, Beijing; ${ }^{(b)}$ Department of Modern Physics, University of Science and Technology of China, Anhui; ${ }^{(c)}$ Department of Physics, Nanjing University, Jiangsu; ${ }^{(d)}$ School of Physics, Shandong University, Shandong, China

33: Laboratoire de Physique Corpusculaire, Clermont Université and Université Blaise Pascal and CNRS/IN2P3, Aubiere Cedex, France

34: Nevis Laboratory, Columbia University, Irvington NY, United States of America

${ }^{35}$ : Niels Bohr Institute, University of Copenhagen, Kobenhavn, Denmark

36: ${ }^{(a)}$ INFN Gruppo Collegato di Cosenza; ${ }^{(b)}$ Dipartimento di Fisica, Università della Calabria, Arcavata di Rende, Italy

37: AGH University of Science and Technology, Faculty of Physics and Applied Computer Science, Krakow, Poland

38: The Henryk Niewodniczanski Institute of Nuclear Physics, Polish Academy of Sciences, Krakow, Poland

39: Physics Department, Southern Methodist University, Dallas TX, United States of America 
40: Physics Department, University of Texas at Dallas, Richardson TX, United States of America

41: DESY, Hamburg and Zeuthen, Germany

42: Institut für Experimentelle Physik IV, Technische Universität Dortmund, Dortmund, Germany

43: Institut für Kern- und Teilchenphysik, Technical University Dresden, Dresden, Germany

44: Department of Physics, Duke University, Durham NC, United States of America

45. SUPA - School of Physics and Astronomy, University of Edinburgh, Edinburgh, United Kingdom

46: Fachhochschule Wiener Neustadt, Johannes Gutenbergstrasse 32700 Wiener Neustadt, Austria

47: INFN Laboratori Nazionali di Frascati, Frascati, Italy

48: Fakultät für Mathematik und Physik, Albert-Ludwigs-Universität, Freiburg i.Br., Germany

49: Section de Physique, Université de Genève, Geneva, Switzerland

50: ${ }^{(a)}$ INFN Sezione di Genova; ${ }^{(b)}$ Dipartimento di Fisica, Università di Genova, Genova, Italy

${ }^{51}{ }^{(a)}$ E.Andronikashvili Institute of Physics, Tbilisi State University, Tbilisi; ${ }^{(b)}$ High Energy Physics Institute, Tbilisi State University, Tbilisi, Georgia

52: II Physikalisches Institut, Justus-Liebig-Universität Giessen, Giessen, Germany

53: SUPA - School of Physics and Astronomy, University of Glasgow, Glasgow, United Kingdom

54: II Physikalisches Institut, Georg-August-Universität, Göttingen, Germany

55: Laboratoire de Physique Subatomique et de Cosmologie, Université Joseph Fourier and CNRS/IN2P3 and Institut National Polytechnique de Grenoble, Grenoble, France

56: Department of Physics, Hampton University, Hampton VA, United States of America

57: Laboratory for Particle Physics and Cosmology, Harvard University, Cambridge MA, United States of America

58: ${ }^{(a)}$ Kirchhoff-Institut für Physik, Ruprecht-Karls-Universität Heidelberg, Heidelberg; ${ }^{(b)}$ Physikalisches Institut, Ruprecht-Karls-Universität Heidelberg, Heidelberg; ${ }^{(c)}$ ZITI Institut für technische Informatik, Ruprecht-Karls-Universität Heidelberg, Mannheim, Germany

59: Faculty of Applied Information Science, Hiroshima Institute of Technology, Hiroshima, Japan

60: Department of Physics, Indiana University, Bloomington IN, United States of America

61: Institut für Astro- und Teilchenphysik, Leopold-Franzens-Universität, Innsbruck, Austria

62: University of Iowa, Iowa City IA, United States of America

63: Department of Physics and Astronomy, Iowa State University, Ames IA, United States of America 
64: Joint Institute for Nuclear Research, JINR Dubna, Dubna, Russia

65: KEK, High Energy Accelerator Research Organization, Tsukuba, Japan

66: Graduate School of Science, Kobe University, Kobe, Japan

67: Faculty of Science, Kyoto University, Kyoto, Japan

68: Kyoto University of Education, Kyoto, Japan

69: Department of Physics, Kyushu University, Fukuoka, Japan

70: Instituto de Física La Plata, Universidad Nacional de La Plata and CONICET, La Plata, Argentina

71: Physics Department, Lancaster University, Lancaster, United Kingdom

72: ${ }^{(a)}$ INFN Sezione di Lecce; ${ }^{(b)}$ Dipartimento di Matematica e Fisica, Università del Salento, Lecce, Italy

73: Oliver Lodge Laboratory, University of Liverpool, Liverpool, United Kingdom

74: Department of Physics, Jožef Stefan Institute and University of Ljubljana, Ljubljana, Slovenia

75: School of Physics and Astronomy, Queen Mary University of London, London, United Kingdom

76: Department of Physics, Royal Holloway University of London, Surrey, United Kingdom

77: Department of Physics and Astronomy, University College London, London, United Kingdom

78: Laboratoire de Physique Nucléaire et de Hautes Energies, UPMC and Université Paris-Diderot and CNRS/IN2P3, Paris, France

79: Fysiska institutionen, Lunds universitet, Lund, Sweden

80: Departamento de Fisica Teorica C-15, Universidad Autonoma de Madrid, Madrid, Spain

81: Institut für Physik, Universität Mainz, Mainz, Germany

82: School of Physics and Astronomy, University of Manchester, Manchester, United Kingdom

83: CPPM, Aix-Marseille Université and CNRS/IN2P3, Marseille, France

84: Department of Physics, University of Massachusetts, Amherst MA, United States of America

85: Department of Physics, McGill University, Montreal QC, Canada

86: School of Physics, University of Melbourne, Victoria, Australia

87: Department of Physics, The University of Michigan, Ann Arbor MI, United States of America

88: Department of Physics and Astronomy, Michigan State University, East Lansing MI, United States of America

89: ${ }^{(a)}$ INFN Sezione di Milano; ${ }^{(b)}$ Dipartimento di Fisica, Università di Milano, Milano, Italy

90: B.I. Stepanov Institute of Physics, National Academy of Sciences of Belarus, Minsk, Republic of Belarus

91: National Scientific and Educational Centre for Particle and High Energy Physics, Minsk, Republic of Belarus 
92: Department of Physics, Massachusetts Institute of Technology, Cambridge MA, United States of America

93: Group of Particle Physics, University of Montreal, Montreal QC, Canada

94: P.N. Lebedev Institute of Physics, Academy of Sciences, Moscow, Russia

95: Institute for Theoretical and Experimental Physics (ITEP), Moscow, Russia

96: Moscow Engineering and Physics Institute (MEPhI), Moscow, Russia

97: Skobeltsyn Institute of Nuclear Physics, Lomonosov Moscow State University, Moscow, Russia

98: Fakultät für Physik, Ludwig-Maximilians-Universität München, München, Germany

99: Max-Planck-Institut für Physik (Werner-Heisenberg-Institut), München, Germany

100: Nagasaki Institute of Applied Science, Nagasaki, Japan

101: Graduate School of Science, Nagoya University, Nagoya, Japan

102: ${ }^{(a)}$ INFN Sezione di Napoli; ${ }^{(b)}$ Dipartimento di Scienze Fisiche, Università di Napoli, Napoli, Italy

103: Department of Physics and Astronomy, University of New Mexico, Albuquerque NM, United States of America

104: Institute for Mathematics, Astrophysics and Particle Physics, Radboud University Nijmegen/Nikhef, Nijmegen, Netherlands

105: Nikhef National Institute for Subatomic Physics and University of Amsterdam, Amsterdam, Netherlands

106: Department of Physics, Northern Illinois University, DeKalb IL, United States of America

107: Budker Institute of Nuclear Physics, SB RAS, Novosibirsk, Russia

108: Department of Physics, New York University, New York NY, United States of America

109: Ohio State University, Columbus OH, United States of America

110: Faculty of Science, Okayama University, Okayama, Japan

111: Homer L. Dodge Department of Physics and Astronomy, University of Oklahoma, Norman OK, United States of America

112: Department of Physics, Oklahoma State University, Stillwater OK, United States of America

113: Palacký University, RCPTM, Olomouc, Czech Republic

114: Center for High Energy Physics, University of Oregon, Eugene OR, United States of America

115: LAL, Université Paris-Sud and CNRS/IN2P3, Orsay, France

116: Graduate School of Science, Osaka University, Osaka, Japan

117: Department of Physics, University of Oslo, Oslo, Norway

118: Department of Physics, Oxford University, Oxford, United Kingdom

119: ${ }^{(a)}$ INFN Sezione di Pavia; ${ }^{(b)}$ Dipartimento di Fisica, Università di Pavia, Pavia, Italy

120: Department of Physics, University of Pennsylvania, Philadelphia PA, United States of America

121: Petersburg Nuclear Physics Institute, Gatchina, Russia

122: ${ }^{(a)}$ INFN Sezione di Pisa; ${ }^{(b)}$ Dipartimento di Fisica E. Fermi, Università di Pisa, Pisa, Italy 
123: Department of Physics and Astronomy, University of Pittsburgh, Pittsburgh PA, United States of America

124: (a) Laboratorio de Instrumentacao e Fisica Experimental de Particulas - LIP, Lisboa, Portugal; ${ }^{(b)}$ Departamento de Fisica Teorica y del Cosmos and CAFPE, Universidad de Granada, Granada, Spain

125: Institute of Physics, Academy of Sciences of the Czech Republic, Praha, Czech Republic

126: Faculty of Mathematics and Physics, Charles University in Prague, Praha, Czech Republic

127: Czech Technical University in Prague, Praha, Czech Republic

128: State Research Center Institute for High Energy Physics, Protvino, Russia

129: Particle Physics Department, Rutherford Appleton Laboratory, Didcot, United Kingdom

130: Physics Department, University of Regina, Regina SK, Canada

131: Ritsumeikan University, Kusatsu, Shiga, Japan

132: ${ }^{(a)}$ INFN Sezione di Roma I; ${ }^{(b)}$ Dipartimento di Fisica, Università La Sapienza, Roma, Italy

133: ${ }^{(a)}$ INFN Sezione di Roma Tor Vergata; ${ }^{(b)}$ Dipartimento di Fisica, Università di Roma Tor Vergata, Roma, Italy

134: ${ }^{(a)}$ INFN Sezione di Roma Tre; ${ }^{(b)}$ Dipartimento di Fisica, Università Roma Tre, Roma, Italy

135: ${ }^{(a)}$ Faculté des Sciences Ain Chock, Réseau Universitaire de Physique des Hautes Energies - Université Hassan II, Casablanca; ${ }^{(b)}$ Centre National de l'Energie des Sciences Techniques Nucleaires, Rabat; ${ }^{(c)}$ Faculté des Sciences Semlalia, Université Cadi Ayyad, LPHEA-Marrakech; ${ }^{(d)}$ Faculté des Sciences, Université Mohamed Premier and LPTPM, Oujda; ${ }^{(e)}$ Faculté des sciences, Université Mohammed V-Agdal, Rabat, Morocco

136: DSM/IRFU (Institut de Recherches sur les Lois Fondamentales de l'Univers), CEA Saclay (Commissariat a l'Energie Atomique), Gif-sur-Yvette, France

137: Santa Cruz Institute for Particle Physics, University of California Santa Cruz, Santa Cruz CA, United States of America

138: Department of Physics, University of Washington, Seattle WA, United States of America

139: Department of Physics and Astronomy, University of Sheffield, Sheffield, United Kingdom

140: Department of Physics, Shinshu University, Nagano, Japan

141: Fachbereich Physik, Universität Siegen, Siegen, Germany

142: Department of Physics, Simon Fraser University, Burnaby BC, Canada

143: SLAC National Accelerator Laboratory, Stanford CA, United States of America

144: ${ }^{(a)}$ Faculty of Mathematics, Physics \& Informatics, Comenius University, Bratislava;

(b) Department of Subnuclear Physics, Institute of Experimental Physics of the Slovak Academy of Sciences, Kosice, Slovak Republic

145: ${ }^{(a)}$ Department of Physics, University of Johannesburg, Johannesburg; ${ }^{(b)}$ School of Physics, University of the Witwatersrand, Johannesburg, South Africa 
146: ${ }^{(a)}$ Department of Physics, Stockholm University; ${ }^{(b)}$ The Oskar Klein Centre, Stockholm, Sweden

147: Physics Department, Royal Institute of Technology, Stockholm, Sweden

148: Departments of Physics \& Astronomy and Chemistry, Stony Brook University, Stony Brook NY, United States of America

149: Department of Physics and Astronomy, University of Sussex, Brighton, United Kingdom

150: School of Physics, University of Sydney, Sydney, Australia

151: Institute of Physics, Academia Sinica, Taipei, Taiwan

152: Department of Physics, Technion: Israel Institute of Technology, Haifa, Israel

153: Raymond and Beverly Sackler School of Physics and Astronomy, Tel Aviv University, Tel Aviv, Israel

154: Department of Physics, Aristotle University of Thessaloniki, Thessaloniki, Greece

155: International Center for Elementary Particle Physics and Department of Physics, The University of Tokyo, Tokyo, Japan

156: Graduate School of Science and Technology, Tokyo Metropolitan University, Tokyo, Japan

157: Department of Physics, Tokyo Institute of Technology, Tokyo, Japan

158: Department of Physics, University of Toronto, Toronto ON, Canada

159: ${ }^{(a)}$ TRIUMF, Vancouver BC; ${ }^{(b)}$ Department of Physics and Astronomy, York University, Toronto ON, Canada

160: Institute of Pure and Applied Sciences, University of Tsukuba,1-1-1 Tennodai,Tsukuba, Ibaraki 305-8571, Japan

161. Science and Technology Center, Tufts University, Medford MA, United States of America

162: Centro de Investigaciones, Universidad Antonio Narino, Bogota, Colombia

163: Department of Physics and Astronomy, University of California Irvine, Irvine CA, United States of America

164: ${ }^{(a)}$ INFN Gruppo Collegato di Udine; ${ }^{(b)}$ ICTP, Trieste; ${ }^{(c)}$ Dipartimento di Chimica, Fisica e Ambiente, Università di Udine, Udine, Italy

165: Department of Physics, University of Illinois, Urbana IL, United States of America

166: Department of Physics and Astronomy, University of Uppsala, Uppsala, Sweden

167: Instituto de Física Corpuscular (IFIC) and Departamento de Física Atómica, Molecular y Nuclear and Departamento de Ingeniería Electrónica and Instituto de Microelectrónica de Barcelona (IMB-CNM), University of Valencia and CSIC, Valencia, Spain

168: Department of Physics, University of British Columbia, Vancouver BC, Canada

169: Department of Physics and Astronomy, University of Victoria, Victoria BC, Canada

170: Department of Physics, University of Warwick, Coventry, United Kingdom

171: Waseda University, Tokyo, Japan

172: Department of Particle Physics, The Weizmann Institute of Science, Rehovot, Israel

173: Department of Physics, University of Wisconsin, Madison WI, United States of America 
174: Fakultät für Physik und Astronomie, Julius-Maximilians-Universität, Würzburg, Germany

175: Fachbereich C Physik, Bergische Universität Wuppertal, Wuppertal, Germany

176: Department of Physics, Yale University, New Haven CT, United States of America

177: Yerevan Physics Institute, Yerevan, Armenia

178: Domaine scientifique de la Doua, Centre de Calcul CNRS/IN2P3, Villeurbanne Cedex, France

${ }^{a}$ : Also at Laboratorio de Instrumentacao e Fisica Experimental de Particulas - LIP, Lisboa, Portugal

${ }^{b}$ : Also at Faculdade de Ciencias and CFNUL, Universidade de Lisboa, Lisboa, Portugal

c: Also at Particle Physics Department, Rutherford Appleton Laboratory, Didcot, United Kingdom

$d$ : Also at TRIUMF, Vancouver BC, Canada

${ }^{e}$ : Also at Department of Physics, California State University, Fresno CA, United States of America

$f$ : Also at Novosibirsk State University, Novosibirsk, Russia

$g$ : Also at Fermilab, Batavia IL, United States of America

$h$ : Also at Department of Physics, University of Coimbra, Coimbra, Portugal

${ }^{i}$ : Also at Department of Physics, UASLP, San Luis Potosi, Mexico

j: Also at Università di Napoli Parthenope, Napoli, Italy

${ }^{k}$ : Also at Institute of Particle Physics (IPP), Canada

${ }^{l}$ : Also at Department of Physics, Middle East Technical University, Ankara, Turkey

$m$ : Also at Louisiana Tech University, Ruston LA, United States of America

${ }^{n}$ : Also at Dep Fisica and CEFITEC of Faculdade de Ciencias e Tecnologia, Universidade Nova de Lisboa, Caparica, Portugal

${ }^{o}$ : Also at Department of Physics and Astronomy, University College London, London, United Kingdom

p: Also at Group of Particle Physics, University of Montreal, Montreal QC, Canada

$q$ : Also at Department of Physics, University of Cape Town, Cape Town, South Africa

$r$ : Also at Institute of Physics, Azerbaijan Academy of Sciences, Baku, Azerbaijan

${ }^{s}$ : Also at Institut für Experimentalphysik, Universität Hamburg, Hamburg, Germany

${ }^{t}$ : Also at Manhattan College, New York NY, United States of America

${ }^{u}$ : Also at School of Physics, Shandong University, Shandong, China

v: Also at CPPM, Aix-Marseille Université and CNRS/IN2P3, Marseille, France

w: Also at School of Physics and Engineering, Sun Yat-sen University, Guanzhou, China

$x$ : Also at Academia Sinica Grid Computing, Institute of Physics, Academia Sinica, Taipei, Taiwan

${ }^{y}$ : Also at Dipartimento di Fisica, Università La Sapienza, Roma, Italy

z: Also at DSM/IRFU (Institut de Recherches sur les Lois Fondamentales de l'Univers), CEA Saclay (Commissariat a l'Energie Atomique), Gif-sur-Yvette, France

aa: Also at section de Physique, Université de Genève, Geneva, Switzerland

${ }^{a b}$ : Also at Departamento de Fisica, Universidade de Minho, Braga, Portugal 
${ }^{a c}$ : Also at Department of Physics and Astronomy, University of South Carolina, Columbia SC, United States of America

${ }^{a d}$ : Also at Institute for Particle and Nuclear Physics, Wigner Research Centre for Physics, Budapest, Hungary

ae: Also at California Institute of Technology, Pasadena CA, United States of America

af: Also at Institute of Physics, Jagiellonian University, Krakow, Poland

ag: Also at LAL, Université Paris-Sud and CNRS/IN2P3, Orsay, France

ah: Also at Department of Physics and Astronomy, University of Sheffield, Sheffield, United Kingdom

${ }^{a i}$ : Also at Department of Physics, Oxford University, Oxford, United Kingdom

${ }^{a j}$ : Also at Institute of Physics, Academia Sinica, Taipei, Taiwan

${ }^{a k}$ : Also at Department of Physics, The University of Michigan, Ann Arbor MI, United States of America

*: Deceased 